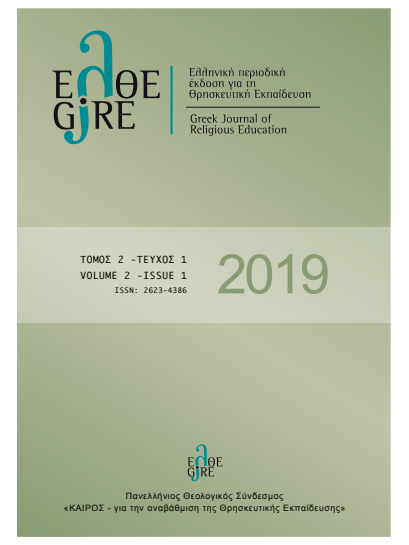

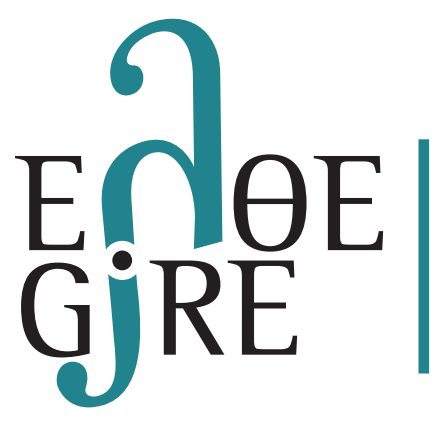

ISSN: 2623-4386.

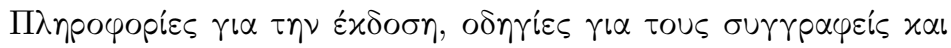

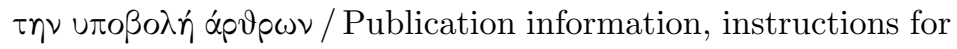
authors and subscription information: http://www.gjre.gr

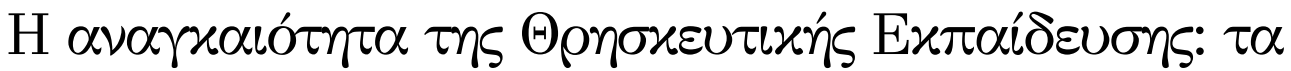

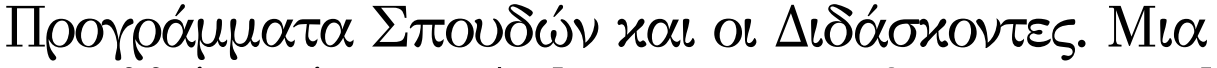

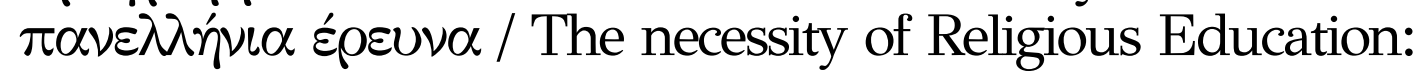 Curricula and Instructors. A pan-Hellenic survey
}

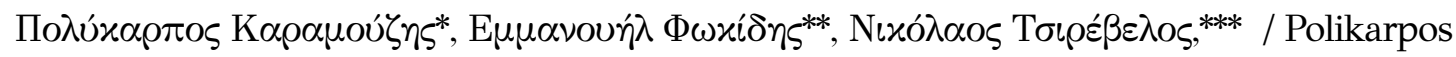
Karamouzis, ${ }^{*}$, Emmanuel Fokides,**, Nikolaos Tsirevelos, ${ }^{* * *}$

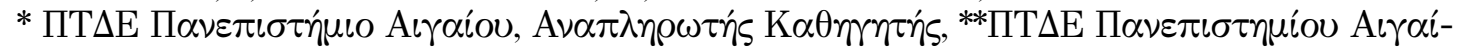

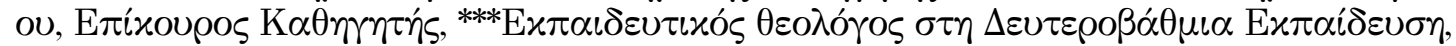

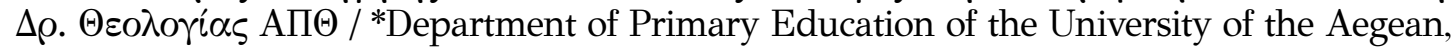
Associate Professor, **Department of Primary Education of the University of the Aegean, Assistant Professor, ${ }^{* * *}$ Teacher of Theology in Secondary Education, Dr of Theology AUTH

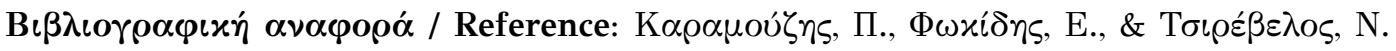
(2019).

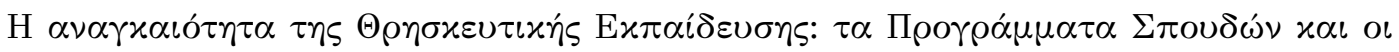

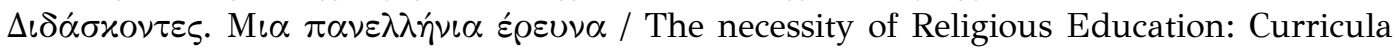

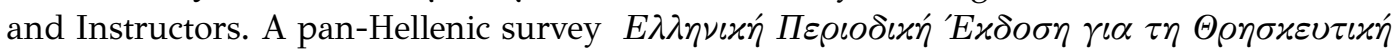

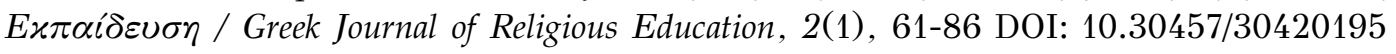

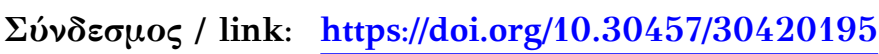

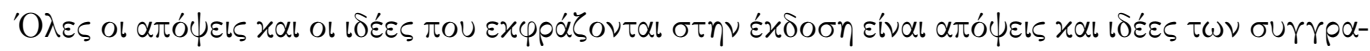

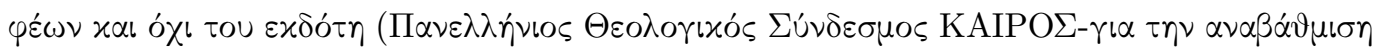

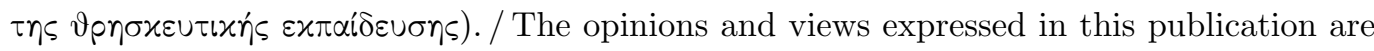
the opinions and views of the authors and not of the publisher (KAIROS - Greek Theological Association for the improvement of the Religious Education).

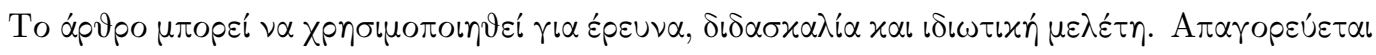

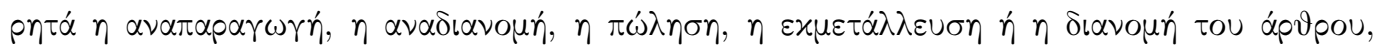

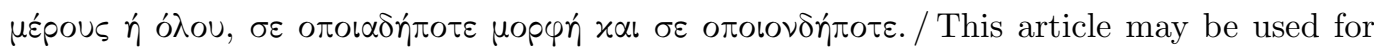
research, teaching, and private study purposes. Any substantial or systematic reproduction, redistribution, reselling, licensing or distribution in any form to anyone is forbidden. 


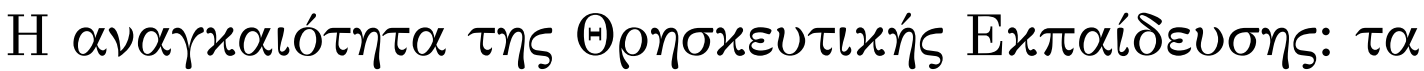

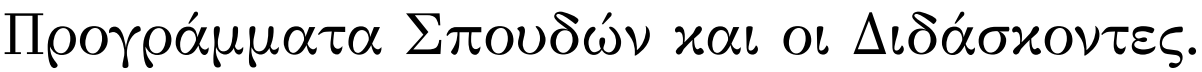

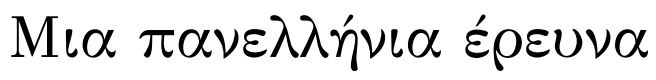

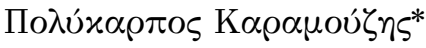

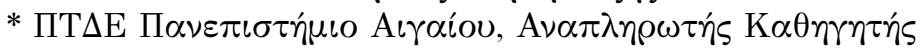 \\ pkaramouzis@rhodes.aegean.gr

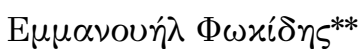

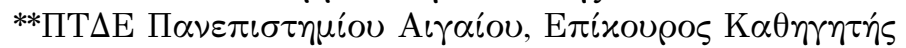 \\ fokides@aegean.gr

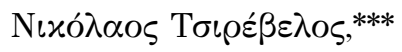

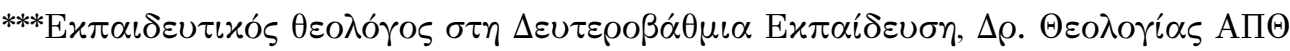 \\ ntsirevelos@hotmail.com
}

\section{The necessity of Religious Education: Curricula and Instructors. A pan-Hellenic survey}

Polikarpos Karamouzis,*

*Department of Primary Education of the University of the Aegean, Associate Professor pkaramouzis@rhodes.aegean.gr

Emmanuel Fokides,**

**Department of Primary Education of the University of the Aegean, Assistant Professor fokides@aegean.gr

Nikolaos Tsirevelos, ${ }^{* * *}$

***Teacher of Theology in Secondary Education, Dr of Theology AUTH ntsirevelos@hotmail.com

\section{$\Pi \varepsilon \rho i ́ \lambda \eta \psi \eta$}

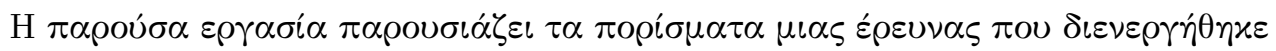

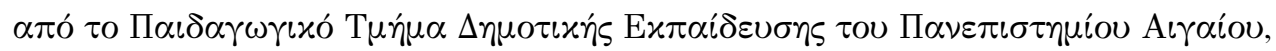

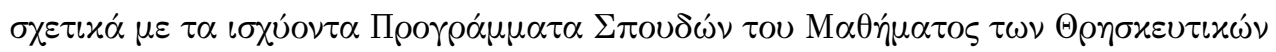

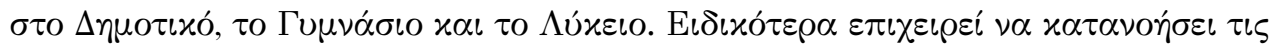

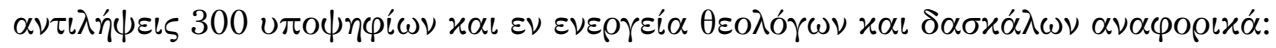

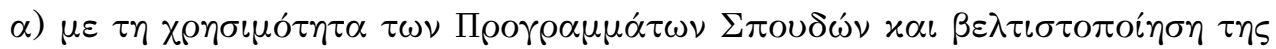

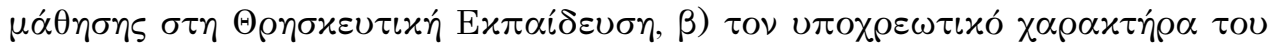

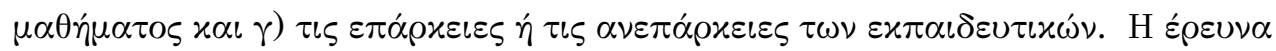

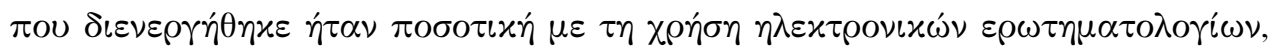

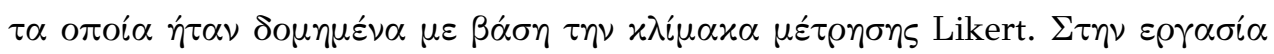

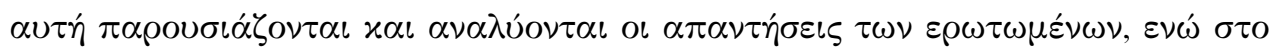




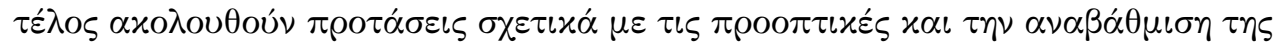

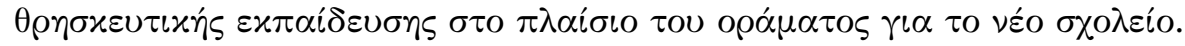

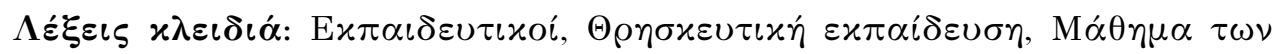
$\Theta \rho \eta \sigma x \varepsilon v \tau \iota x \omega \dot{\nu}, \Pi \rho \circ \gamma \rho \alpha ́ \alpha \mu \alpha \tau \alpha \Sigma \pi o v \delta \omega \dot{\nu}$.

\begin{abstract}
This paper presents the findings of a survey conducted by the Department of Primary Education of the University of the Aegean on the current Religious Education in Primary and Secondary Education. In particular, it attempts to understand the perceptions of 300 candidates and active theologians and teachers regarding: a) the usefulness of curricula and optimization of learning in Religious Education, b) the compulsory or optional nature of the course and c) the teachers' competencies or incompetencies. The survey carried out was quantitative using electronic questionnaires, structured on the basis of the Likert measurement scale. In this paper the answers of the respondents are presented and analyzed, while in the end there are suggestions on the prospects and the upgrading of religious education within the framework of the vision for the new school.
\end{abstract}

Keywords: Curricula, Teachers, Religious Education.

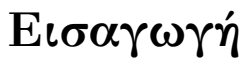

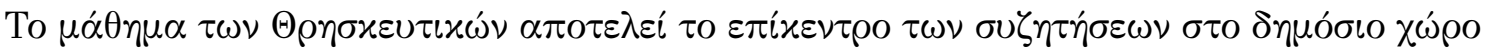

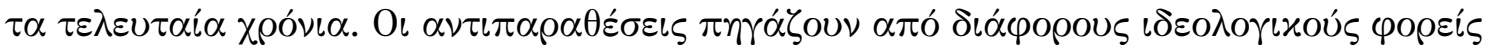

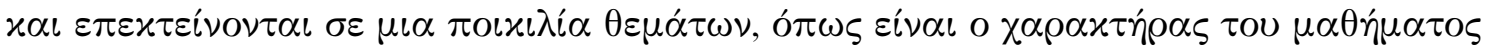

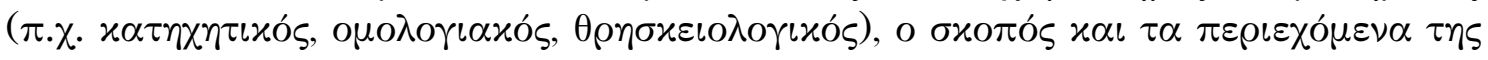

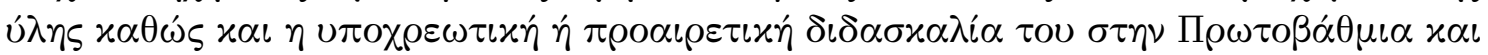

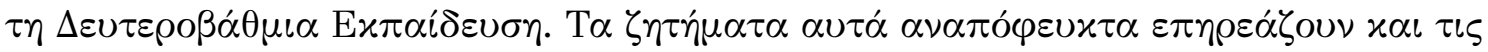

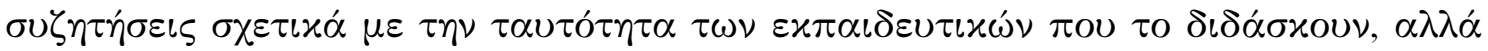

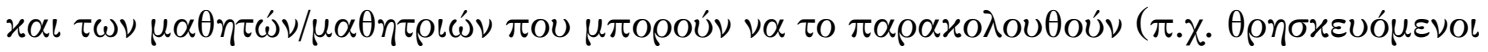

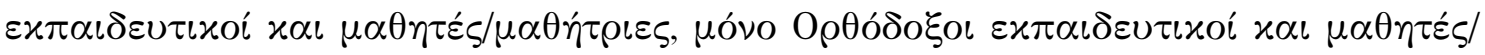
$\mu \alpha \theta \dot{\eta} \tau \rho \iota \varepsilon \varsigma x . \lambda \pi$.).

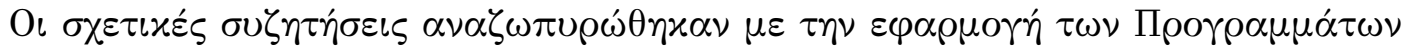

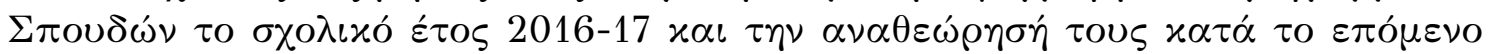

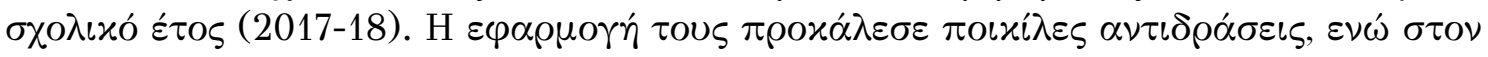

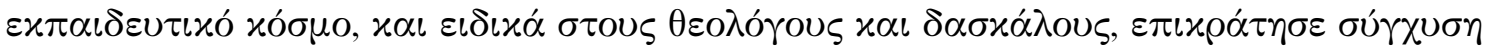

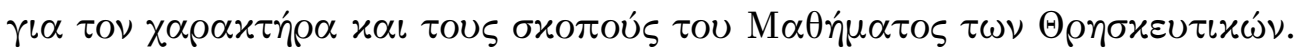

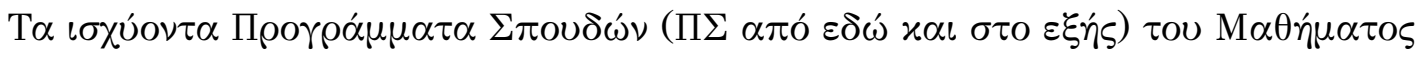

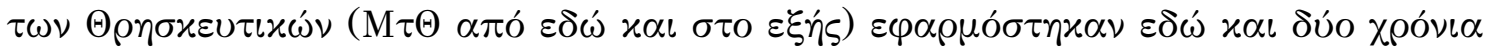

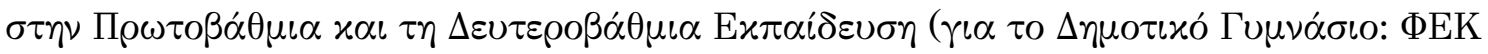

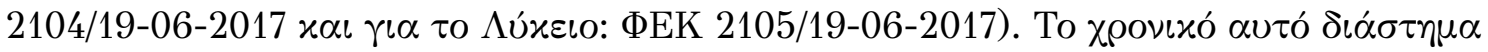

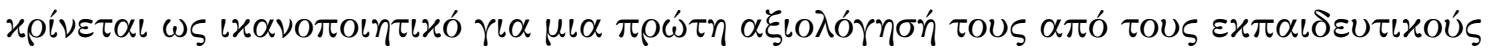

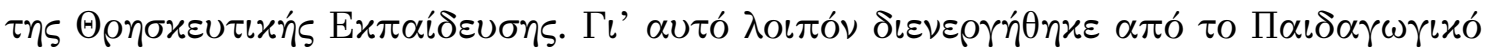

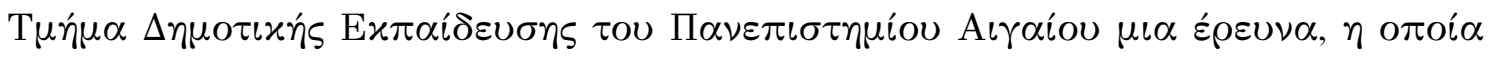




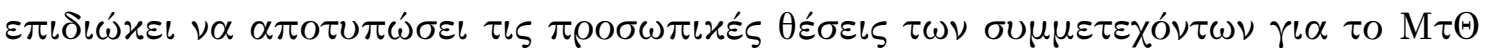

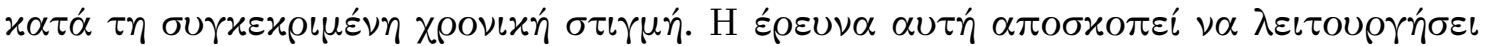

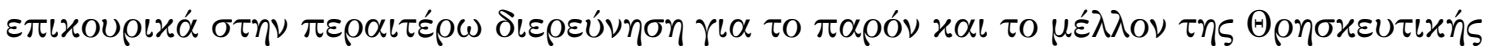

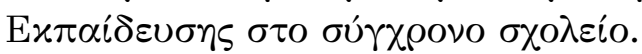

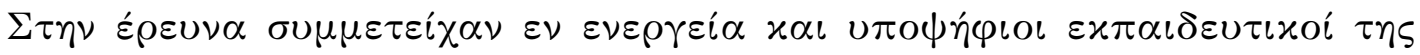

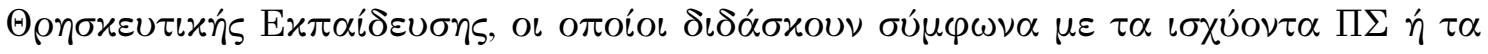

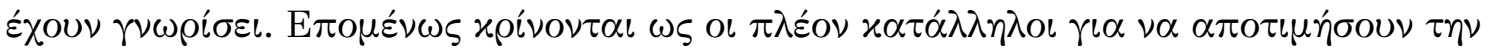

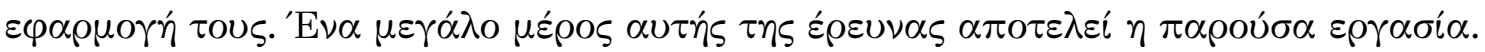

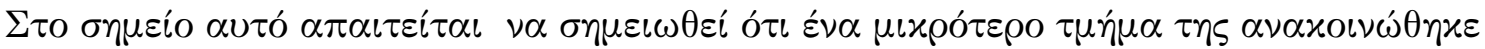

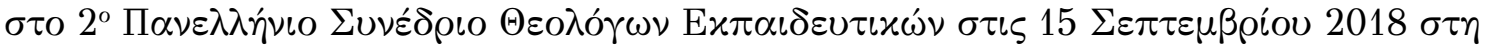

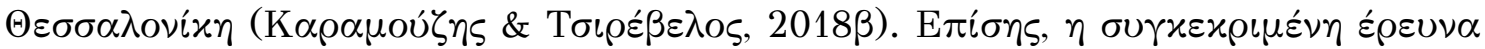

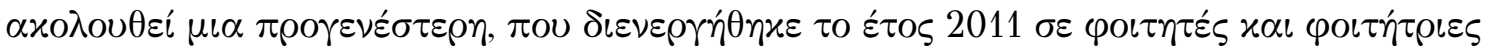

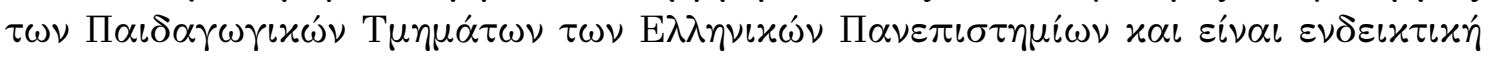

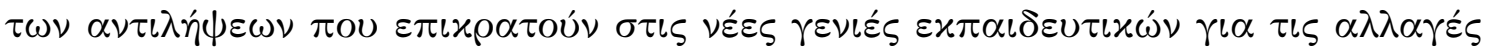

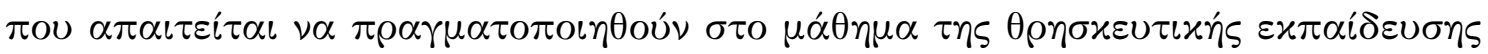

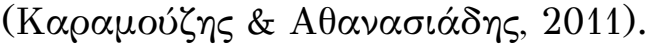

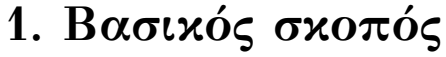

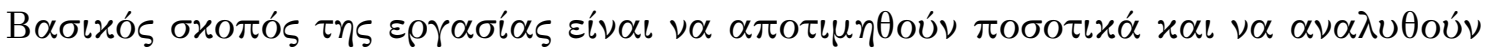

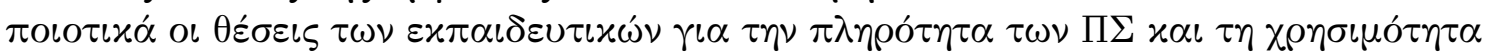

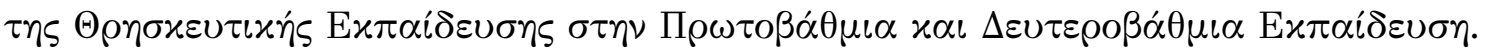

\section{2. Е}

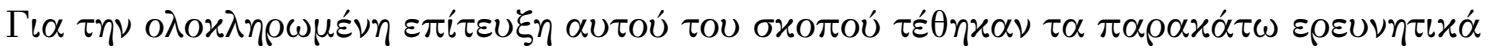

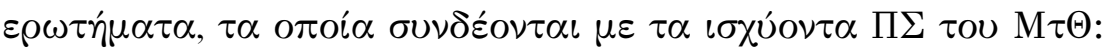

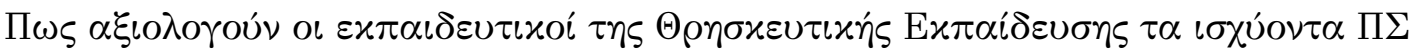

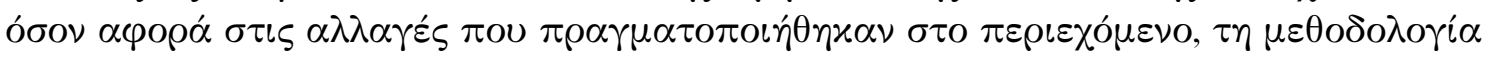

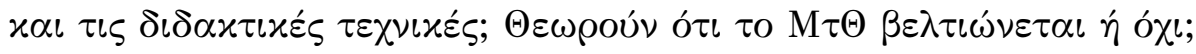

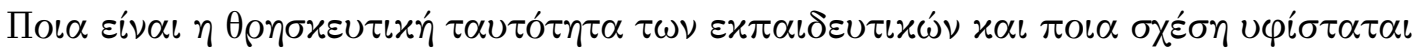

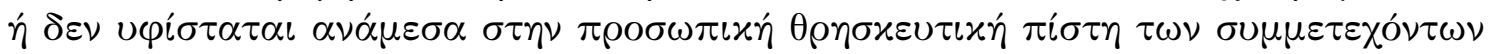

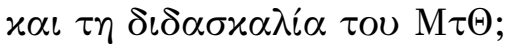

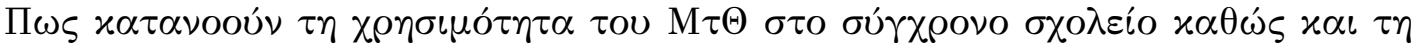

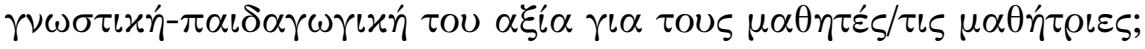

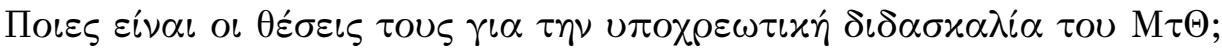

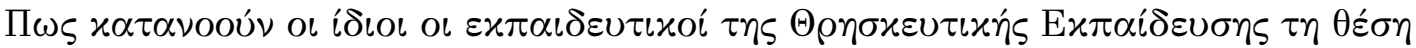

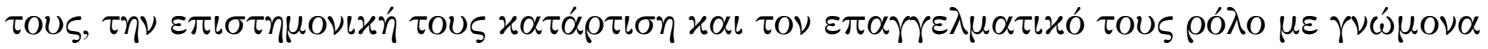
$\tau \alpha \iota \sigma \chi \cup ́ o \nu \tau \alpha \Pi \Sigma$

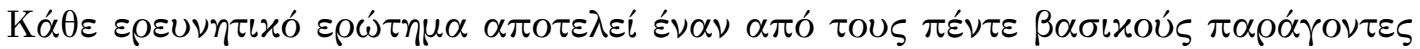

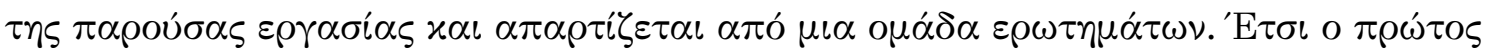

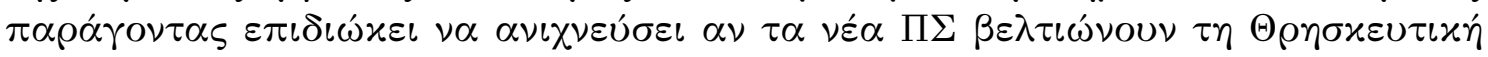

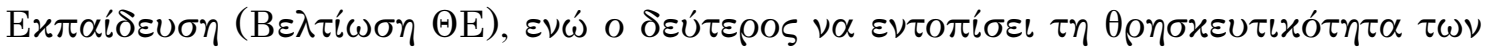




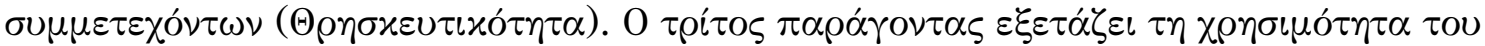

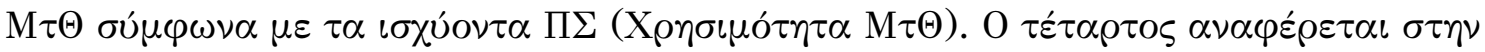

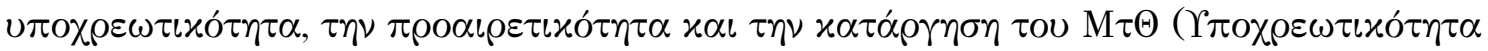

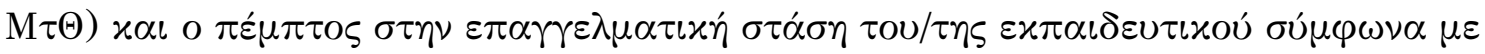

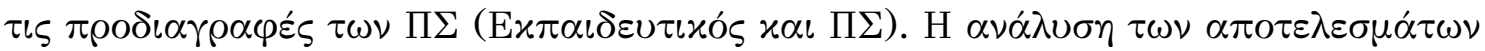

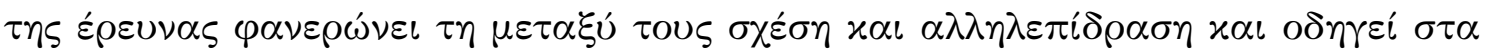

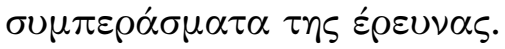

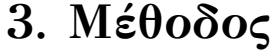

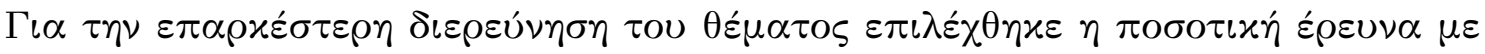

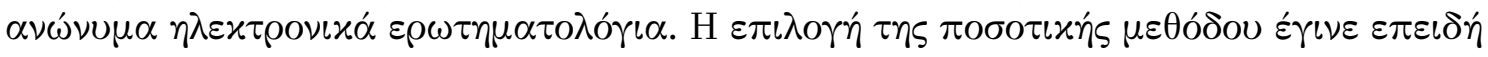

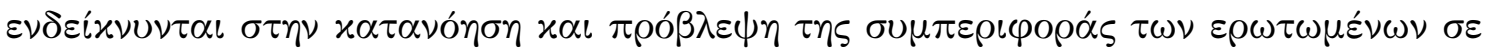

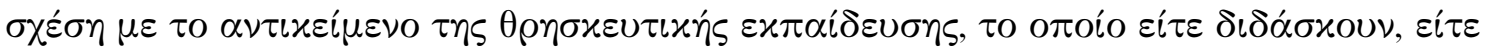

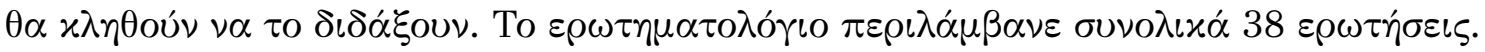

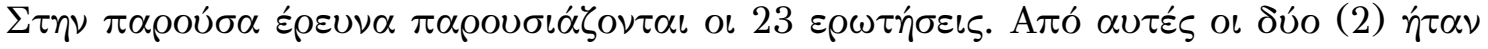

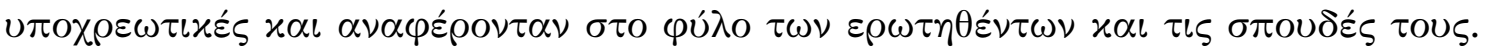

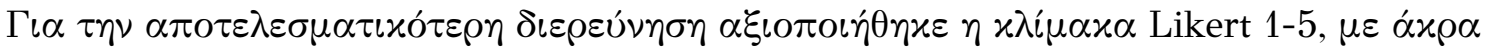

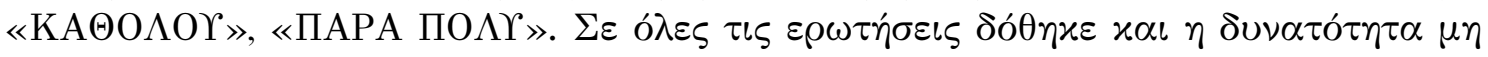
$\alpha \pi \alpha ́ \nu \tau \eta \sigma \eta \varsigma, \mu \varepsilon \tau \eta \nu \varepsilon \pi \iota \lambda o \gamma \dot{\eta} \ll \Delta \mathrm{EN}$ AПANT $\Omega »$.

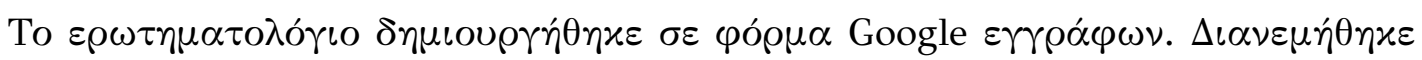

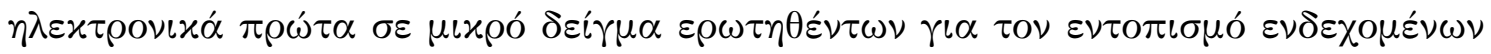

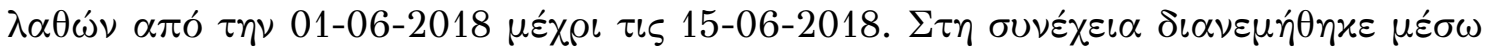

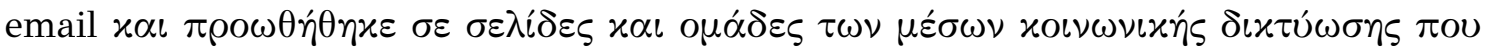

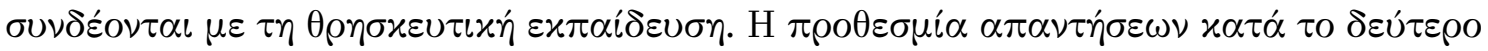

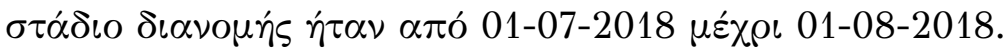

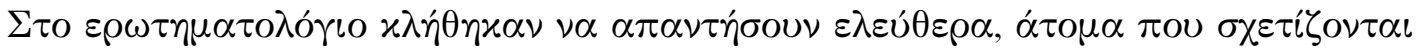

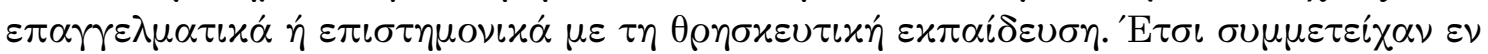

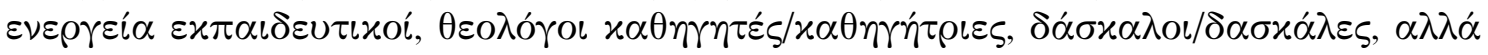

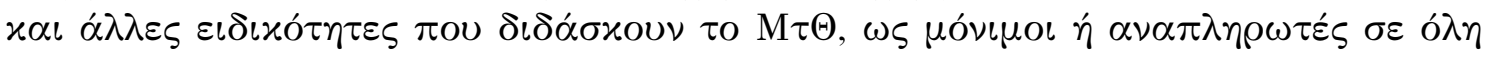

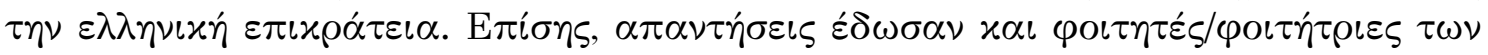

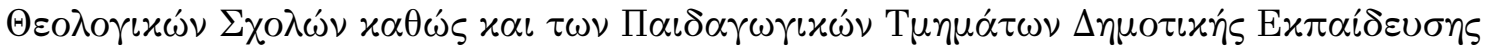

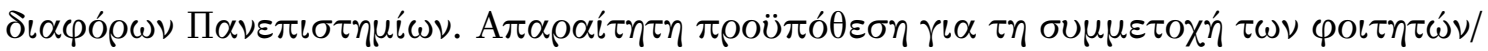

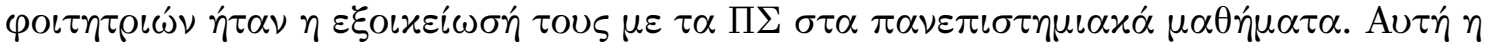

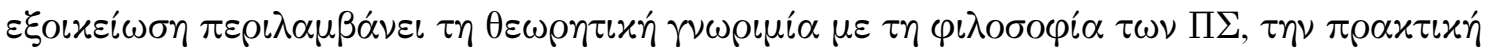

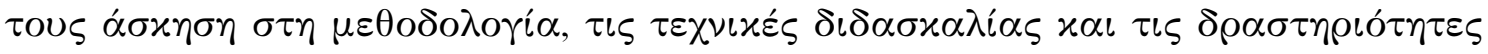

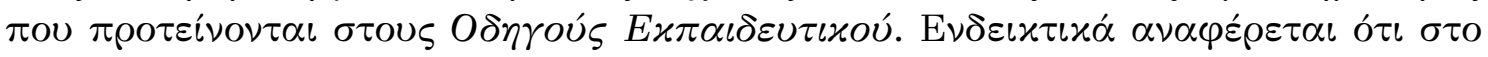

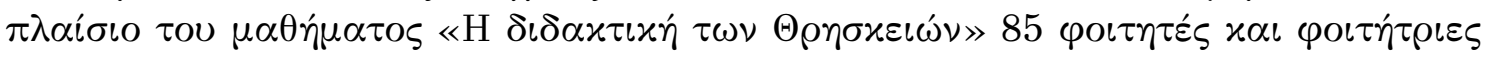

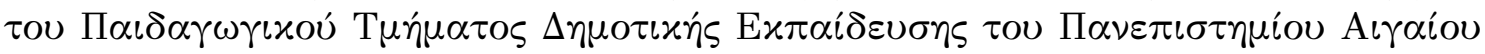

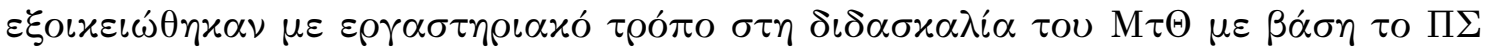

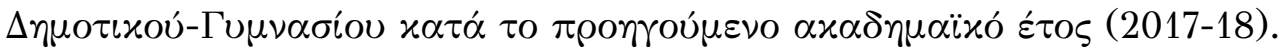

\section{H $\varepsilon \rho \varepsilon v \nu \eta \tau \iota \varkappa \eta ́ ~ \varepsilon \rho \gamma \alpha \sigma i \alpha$}

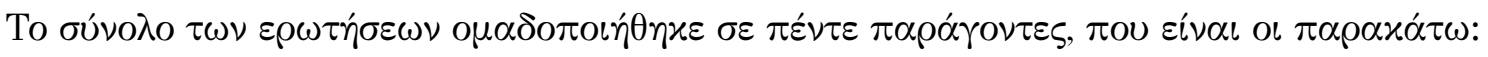




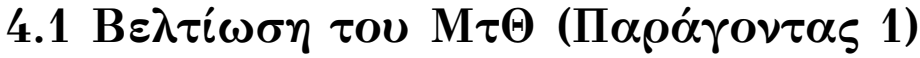

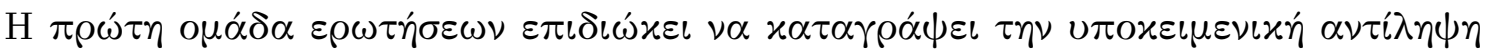

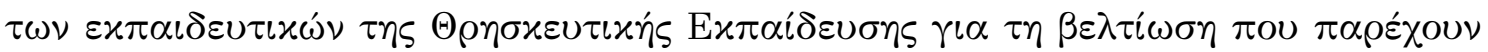

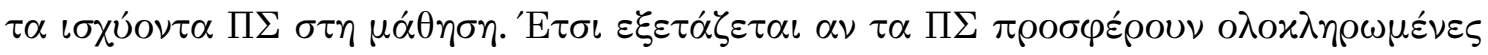

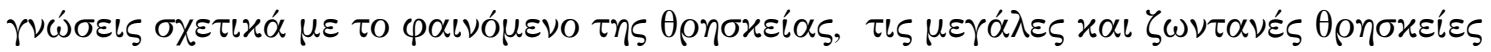

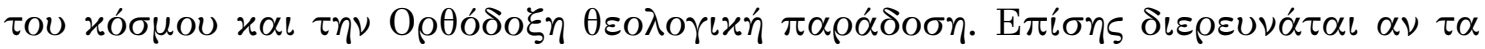

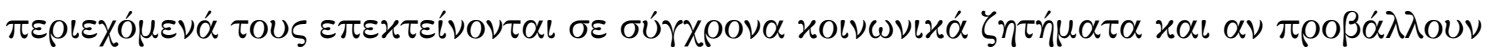

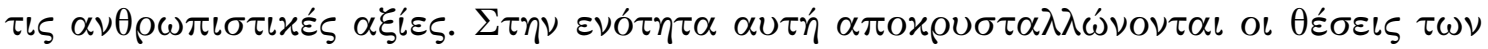

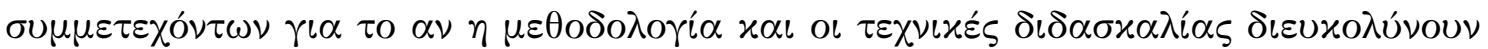

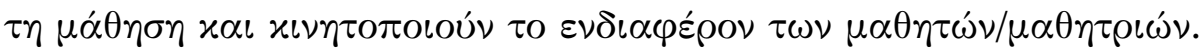

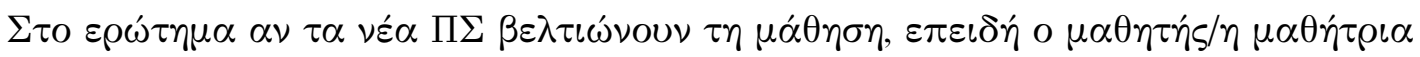

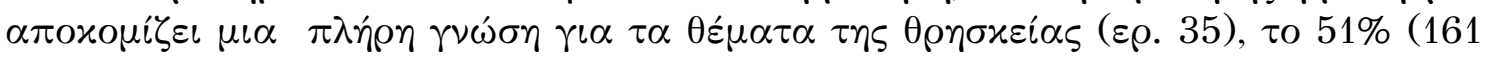

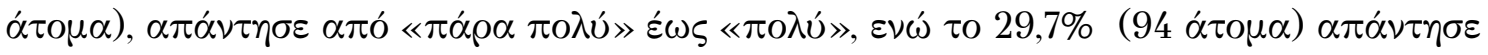

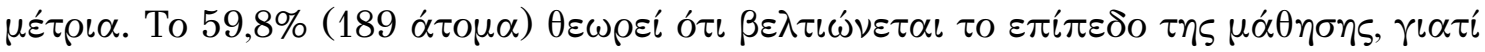

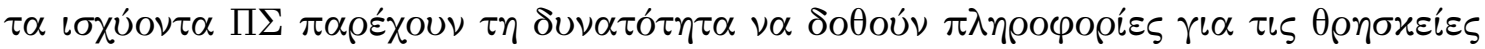

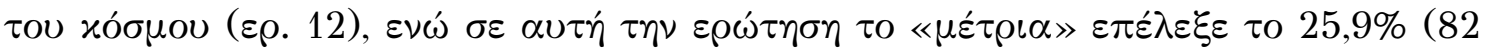

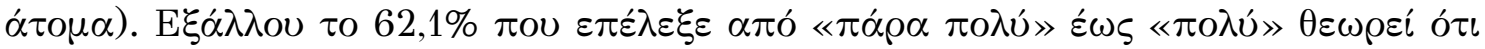

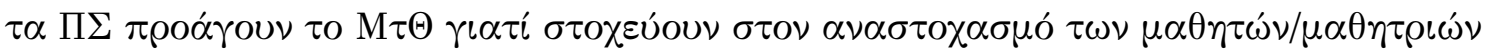

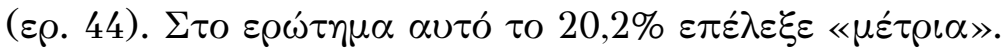

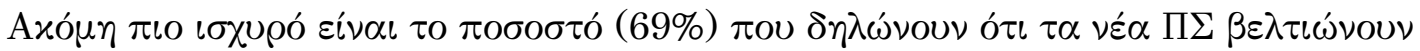

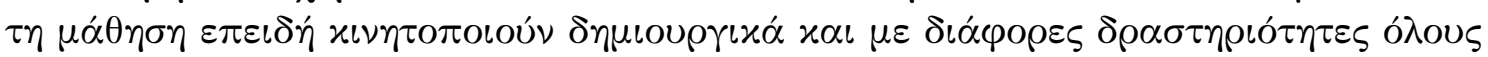

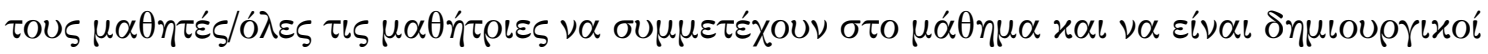

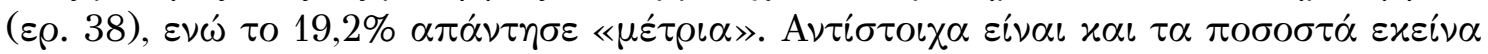

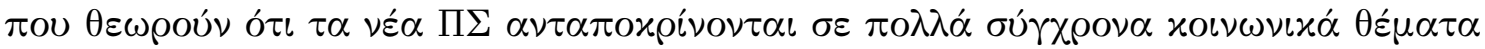

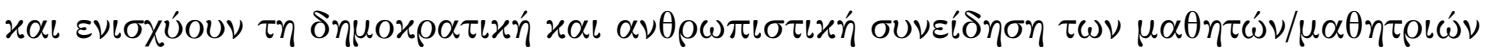

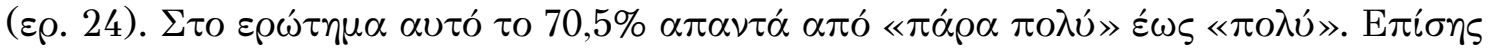

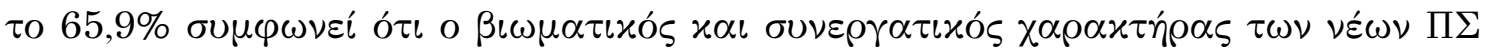

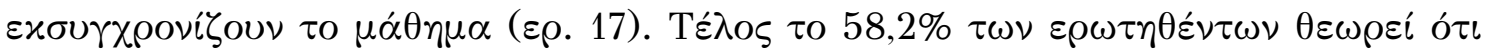

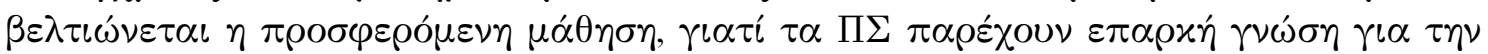

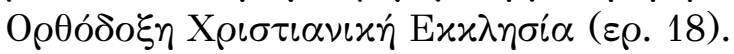

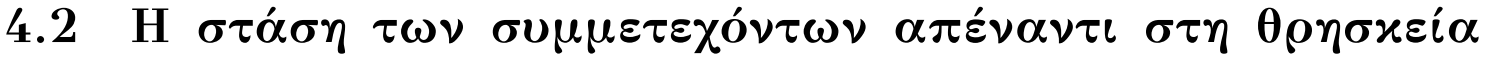

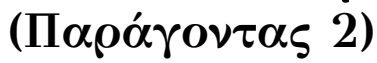

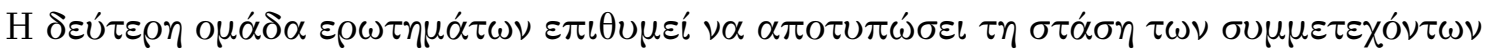

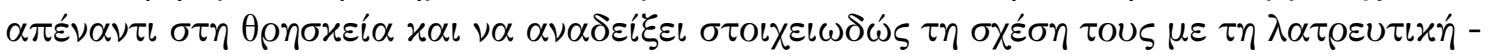

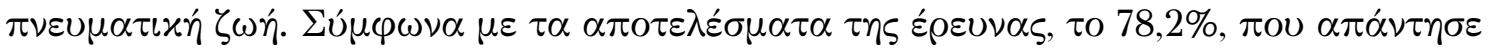

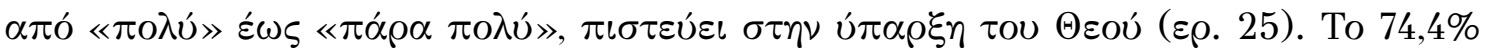

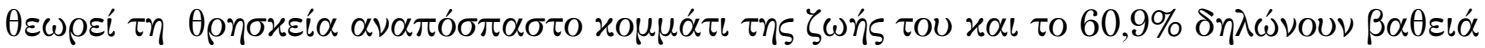

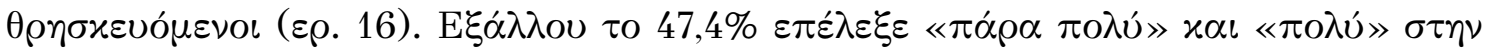

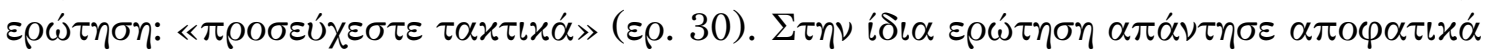

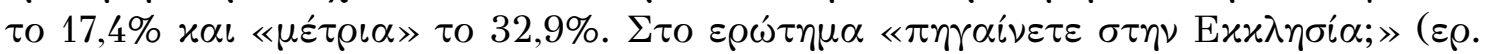

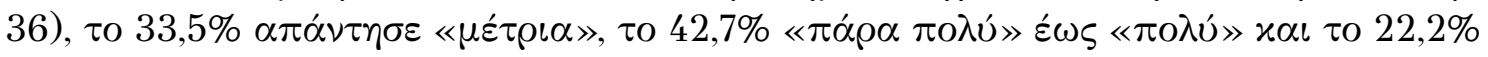

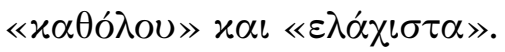




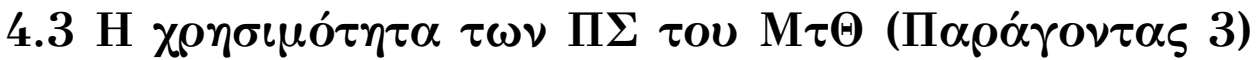

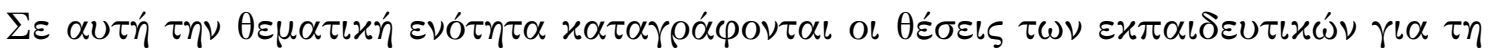

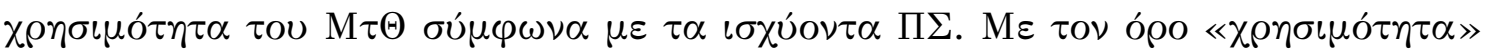

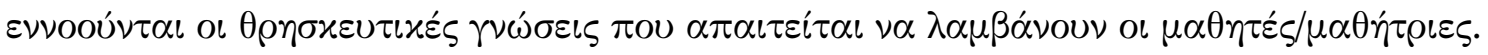

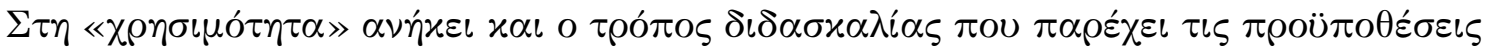

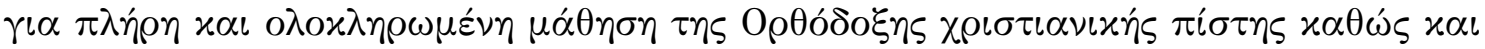

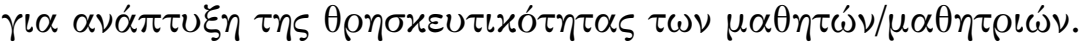

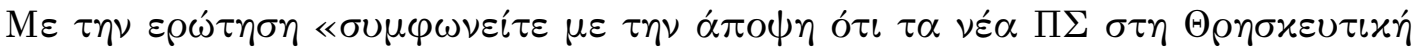

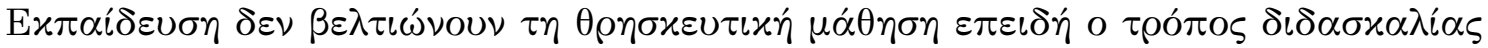

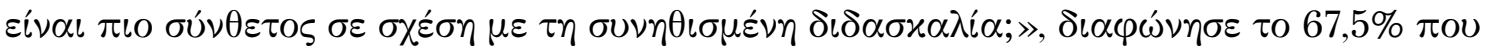

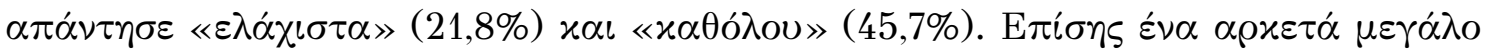

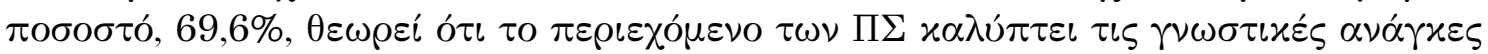

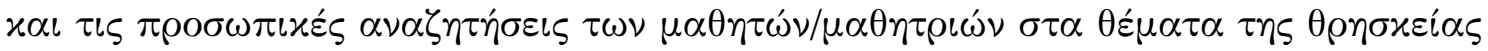

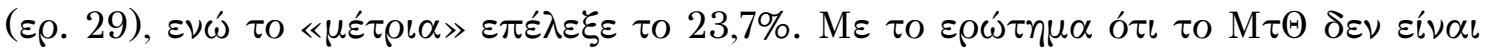

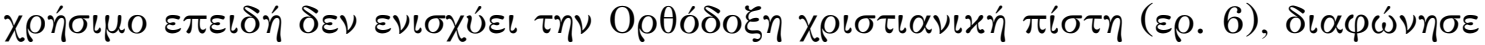

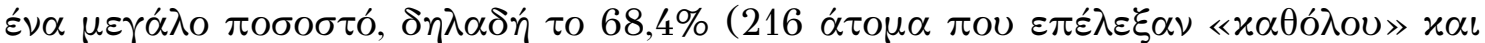

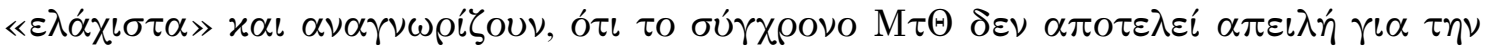

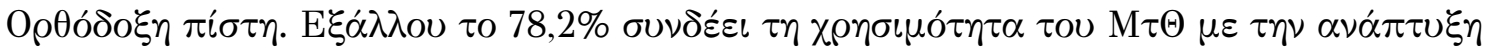

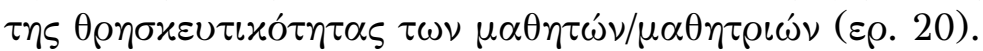

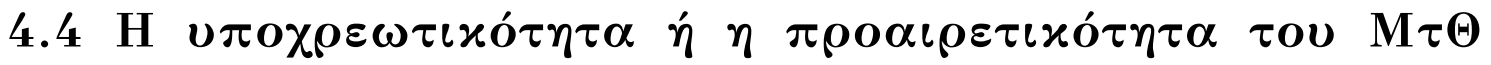

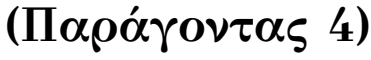

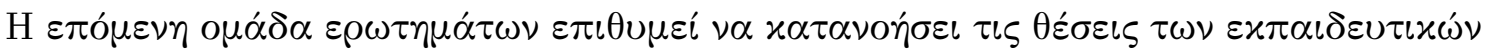

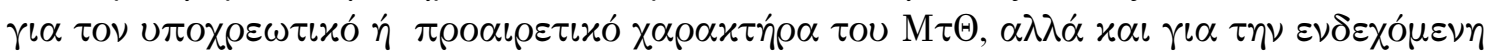

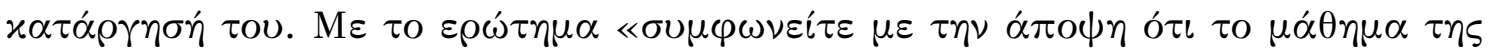

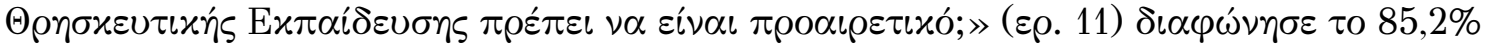

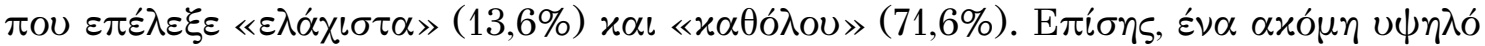

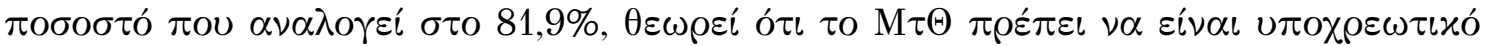

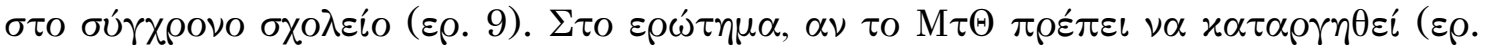

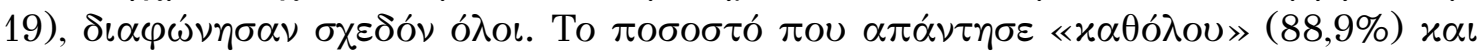

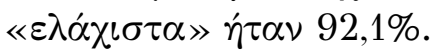

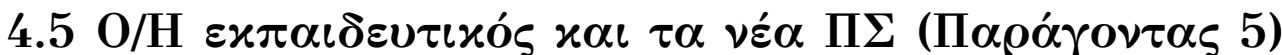

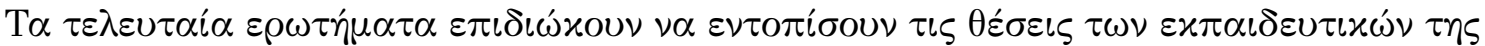

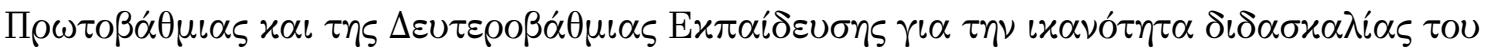

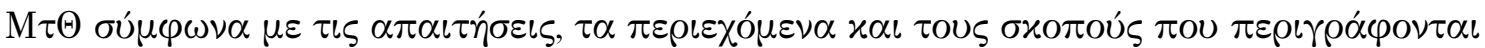

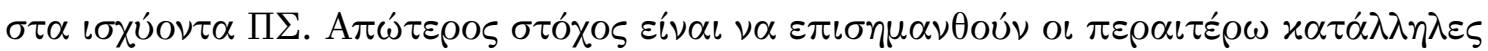

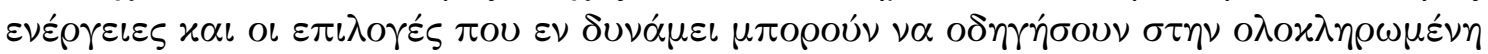
$x \alpha \tau \alpha \dot{\rho} \rho \iota \sigma \eta \tau \omega \nu \varepsilon x \pi \alpha \iota \delta \varepsilon v \tau \iota x \omega \dot{\nu}$.

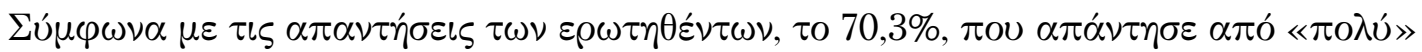

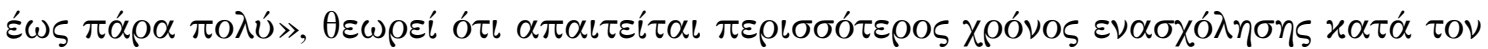

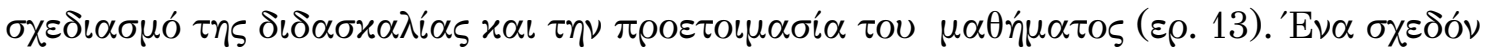




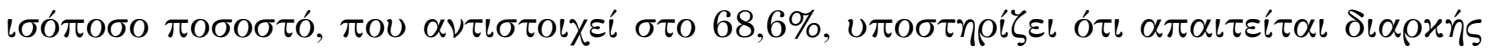

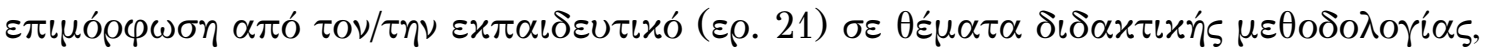

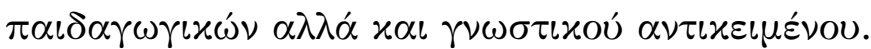

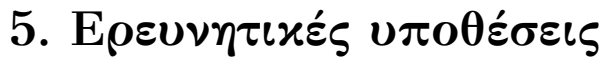

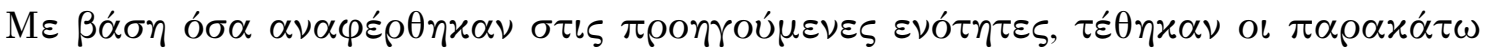

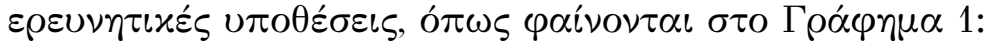

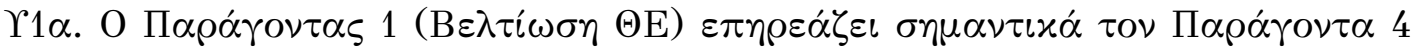
$(\Upsilon \pi 0 \chi \rho \varepsilon \omega \tau \iota x o ́ \tau \eta \tau \alpha \mathrm{M} \tau \Theta)$.

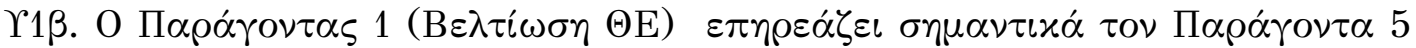

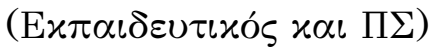

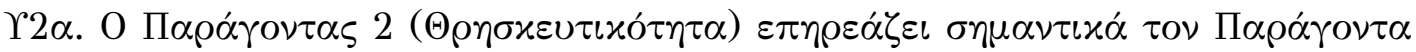
$4(\Upsilon \pi 0 \chi \rho \varepsilon \omega \tau \iota x o ́ \tau \eta \tau \alpha \mathrm{M} \tau \Theta)$.

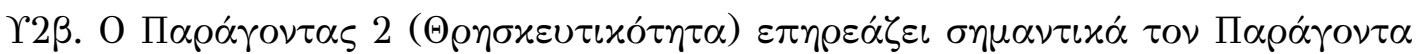

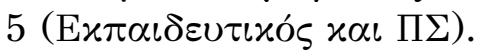

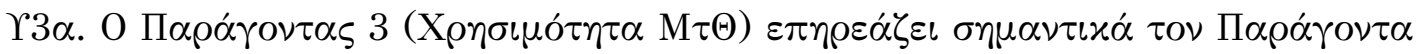
$4(\Upsilon \pi 0 \chi \rho \varepsilon \omega \tau \iota \varkappa o ́ \tau \eta \tau \alpha \mathrm{M} \tau \Theta)$.

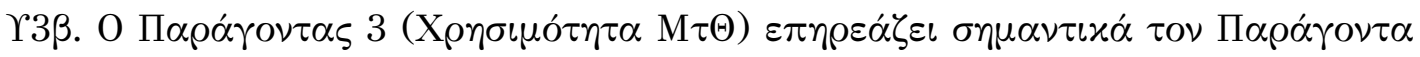
$5(\mathrm{E} \varkappa \pi \alpha \iota \delta \varepsilon v \tau \iota x \circ \iota \mathfrak{x} \varkappa \iota \iota \Pi \Sigma)$.

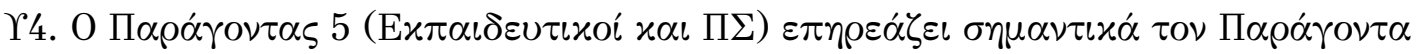
$4(\Upsilon \pi 0 \chi \rho \varepsilon \omega \tau \iota x o ́ \tau \eta \tau \alpha \mathrm{M} \tau \Theta)$.

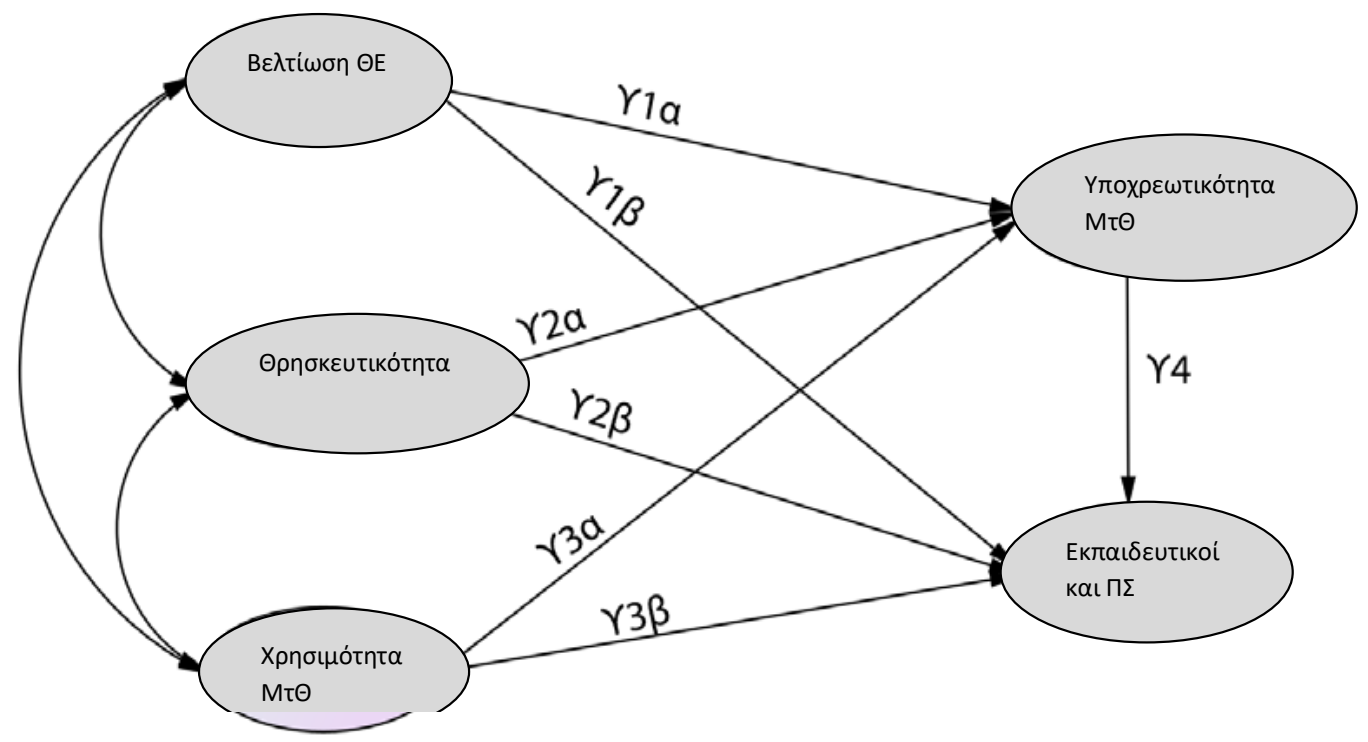

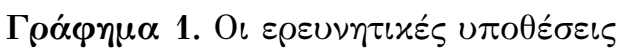




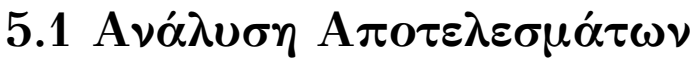

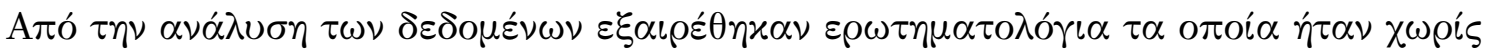

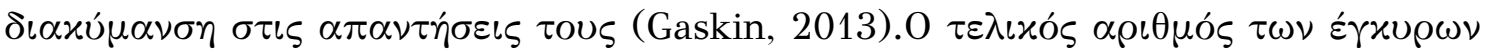

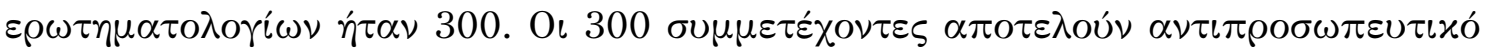

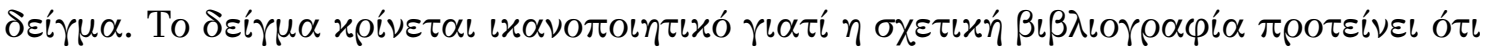

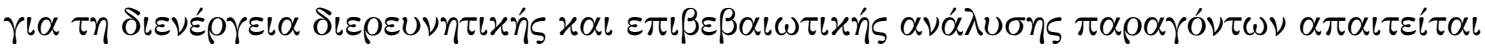

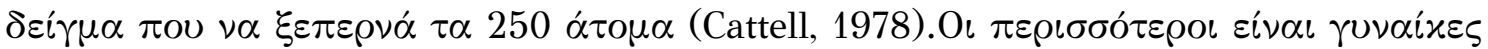

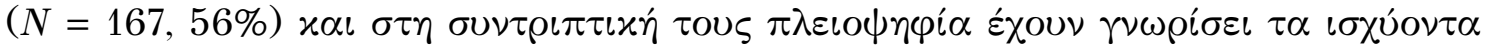

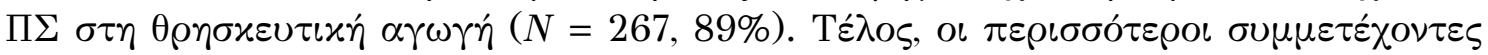

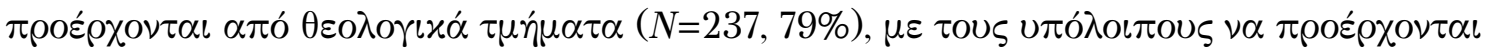

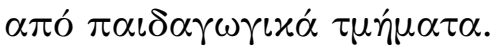

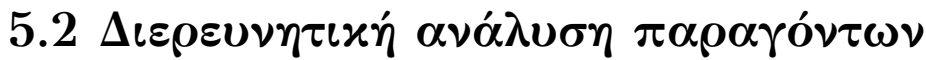

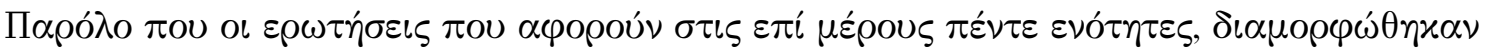

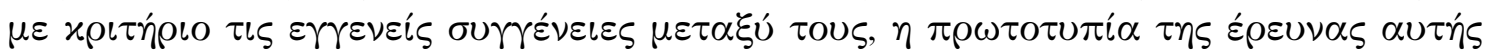

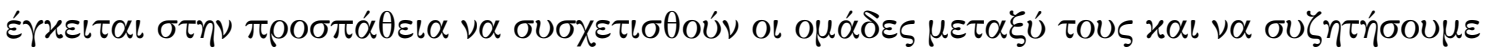

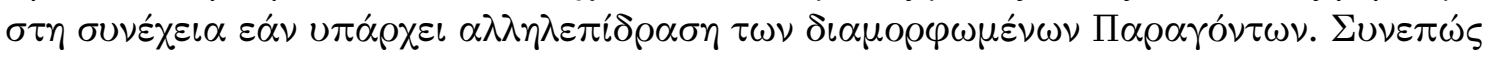

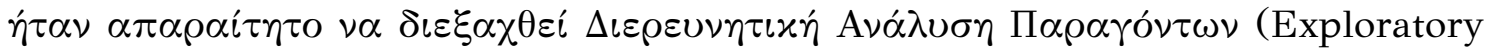

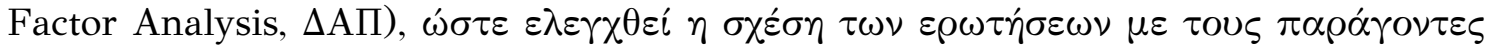

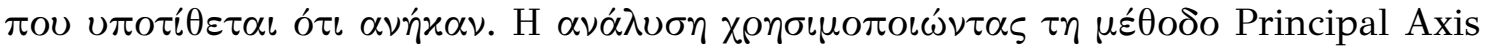

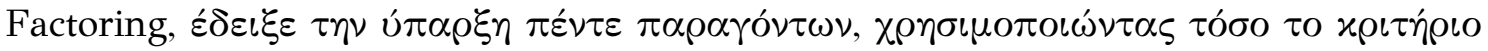

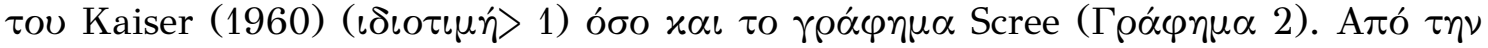

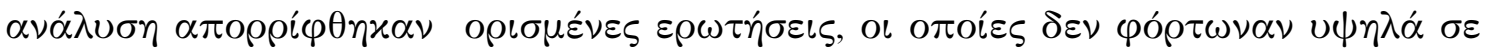

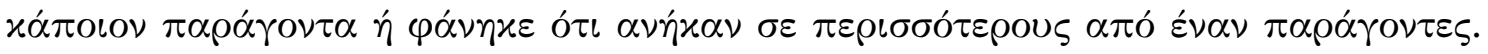

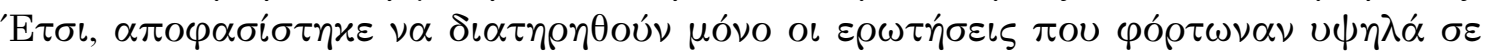

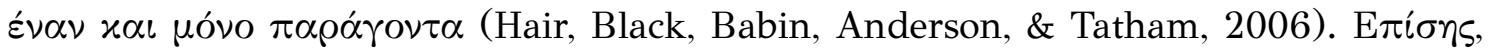

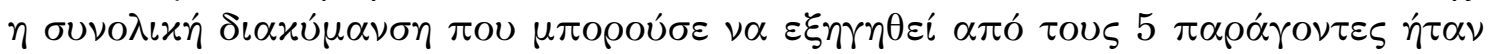

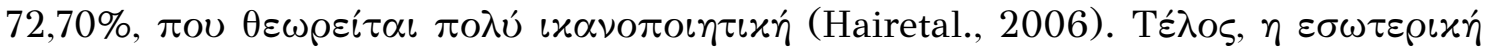

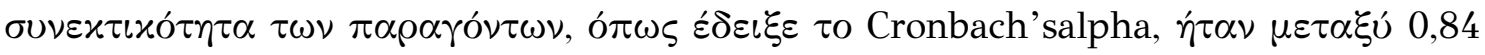

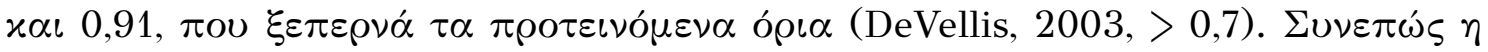

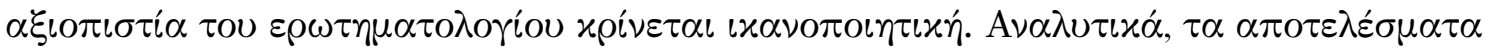

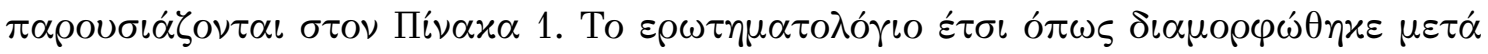

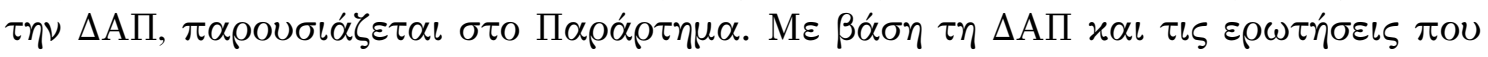

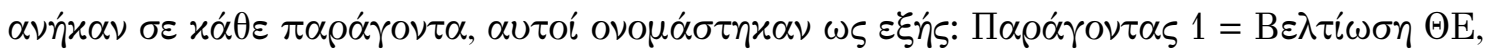

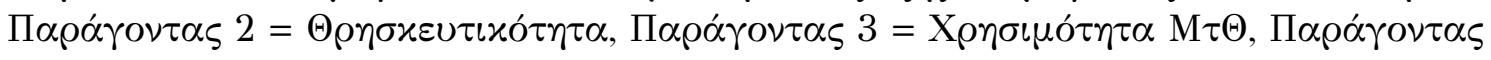

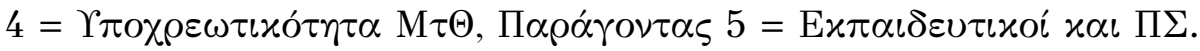




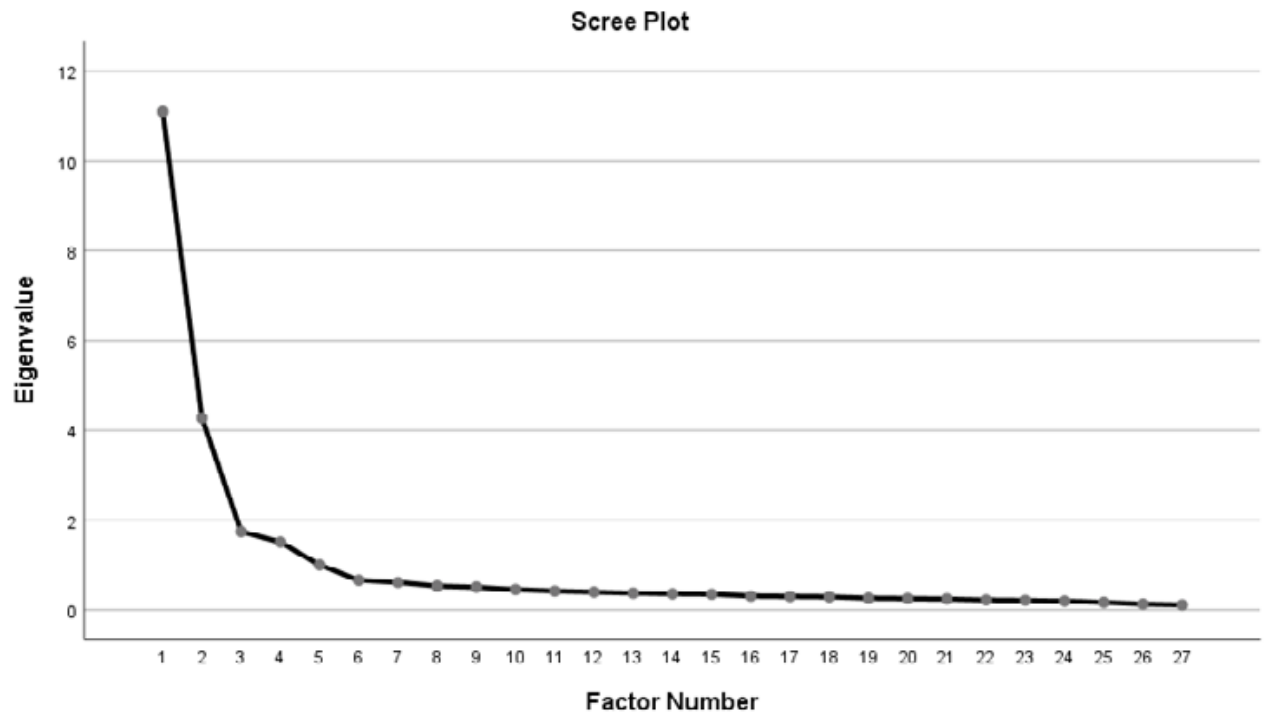

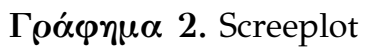




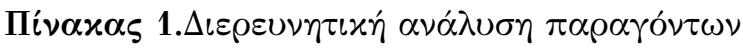

\begin{tabular}{|c|c|c|c|c|c|}
\hline \multicolumn{6}{|c|}{ 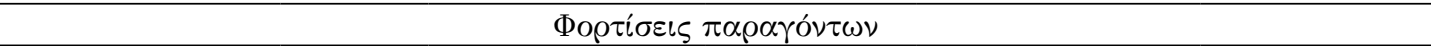 } \\
\hline & & & 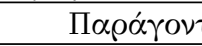 & & \\
\hline & $\begin{array}{c}1 \\
\mathrm{~B} \varepsilon \lambda \tau i \omega \\
\sigma \eta \Theta \mathrm{E}\end{array}$ & $\begin{array}{c}2 \\
\Theta \rho \eta \sigma x \varepsilon v \tau \iota \varkappa o ́ \tau \eta \tau \alpha\end{array}$ & $\begin{array}{c}3 \\
\mathrm{X} \rho \eta \sigma \iota \mu o ́ \tau \eta \tau \alpha \\
\mathrm{M} \tau \Theta\end{array}$ & $\begin{array}{c}4 \\
\Upsilon \pi \sigma \chi \rho \varepsilon \omega \tau \iota \chi o ́ \tau \eta \tau \alpha \\
M \tau \Theta\end{array}$ & $\begin{array}{c}5 \\
\mathrm{E} \varkappa \pi \alpha \iota \delta \varepsilon v \tau \iota \varkappa o i ́ \\
\varkappa \alpha \iota \Pi \Sigma\end{array}$ \\
\hline q37 & 0,988 & & & & \\
\hline q10 & 0,896 & & & & \\
\hline q35 & 0,881 & & & & \\
\hline$q 12$ & 0,829 & & & & \\
\hline q7 & 0,827 & & & & \\
\hline$q 44$ & 0,821 & & & & \\
\hline q38 & 0,819 & & & & \\
\hline q33 & 0,790 & & & & \\
\hline$q^{4}$ & 0,772 & & & & \\
\hline q24 & 0,764 & & & & \\
\hline q17 & 0,753 & & & & \\
\hline$q 40$ & 0,749 & & & & \\
\hline q18 & 0,642 & & & & \\
\hline q30 & & 0,909 & & & \\
\hline q16 & & 0,886 & & & \\
\hline q36 & & 0,852 & & & \\
\hline $\mathrm{q} 25$ & & 0,799 & & & \\
\hline q14 & & 0,642 & & & \\
\hline q26 & & & 0,729 & & \\
\hline q29 & & & 0,712 & & \\
\hline q6 & & & 0,652 & & \\
\hline q20 & & & 0,641 & & \\
\hline q11 & & & & 0,927 & \\
\hline q19 & & & & 0,815 & \\
\hline q9 & & & & 0,655 & \\
\hline q13 & & & & & 0,869 \\
\hline q21 & & & & & 0,768 \\
\hline 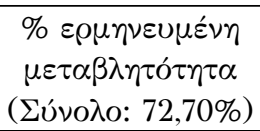 & 41,09 & 15,84 & 6,46 & 5,60 & 3,71 \\
\hline 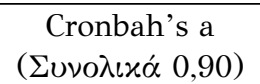 & 0,87 & 0,84 & 0,90 & 0,91 & 0,88 \\
\hline
\end{tabular}

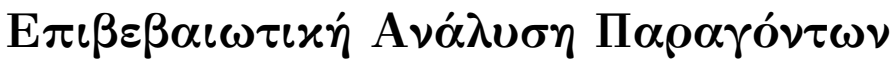

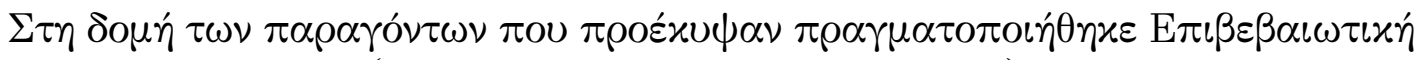

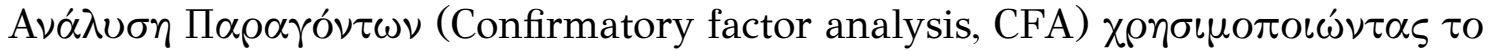

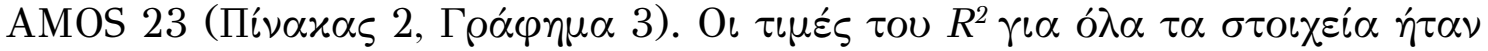

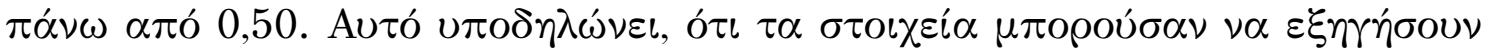




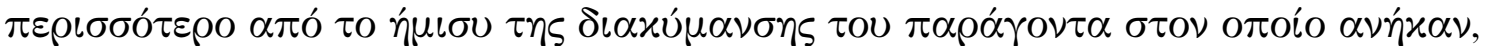

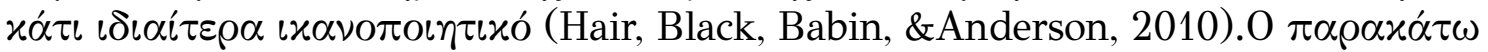

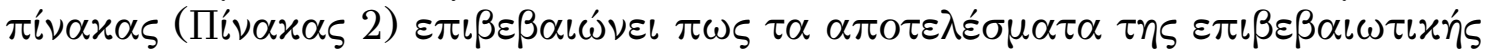

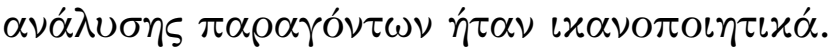

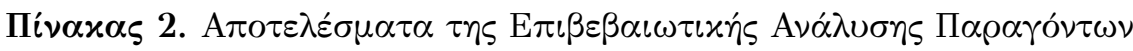

\begin{tabular}{|c|c|c|c|c|}
\hline 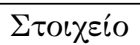 & SE & $t$-value & $R^{2}$ & MEA \\
\hline q37 & 0,878 & - & 0,771 & 0,82 \\
\hline q10 & 0,903 & 23,351 & 0,815 & \\
\hline q35 & 0,785 & 17,712 & 0,616 & \\
\hline $\mathrm{q} 12$ & 0,674 & 13,886 & 0,554 & \\
\hline q7 & 0,868 & 21,444 & 0,754 & \\
\hline$q 44$ & 0,857 & 20,847 & 0,735 & \\
\hline q38 & 0,878 & 25,031 & 0,771 & \\
\hline q33 & 0,810 & 18,726 & 0,656 & \\
\hline$q^{4}$ & 0,772 & 17,223 & 0,597 & \\
\hline $\mathrm{q} 24$ & 0,816 & 21,744 & 0,665 & \\
\hline q17 & 0,879 & 22,058 & 0,773 & \\
\hline $\mathrm{q} 40$ & 0,771 & 17,172 & 0,595 & \\
\hline q18 & 0,738 & 15,966 & 0,545 & \\
\hline q30 & 0,790 & - & 0,624 & 0,81 \\
\hline q16 & 0,853 & 16,013 & 0,728 & \\
\hline q36 & 0,762 & 16,628 & 0,581 & \\
\hline q25 & 0,838 & 15,682 & 0,702 & \\
\hline q14 & 0,817 & 15,206 & 0,667 & \\
\hline q26 & 0,665 & - & 0,542 & 0,71 \\
\hline q29 & 0,700 & 10,009 & 0,590 & \\
\hline $\mathrm{q} 6$ & 0,717 & 10,192 & 0,514 & \\
\hline q20 & 0,763 & 10,651 & 0,583 & \\
\hline q11 & 0,627 & - & 0,593 & 0,77 \\
\hline q19 & 0,614 & 13,156 & 0,577 & \\
\hline q9 & 0,993 & 9,031 & 0,986 & \\
\hline q13 & 0,846 & - & 0,716 & 0,84 \\
\hline q21 & 0,831 & 11,721 & 0,690 & \\
\hline
\end{tabular}

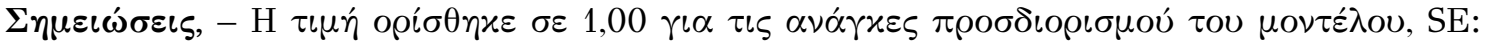

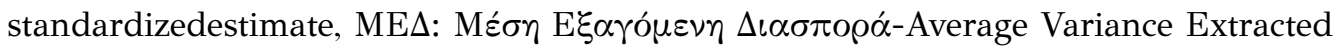




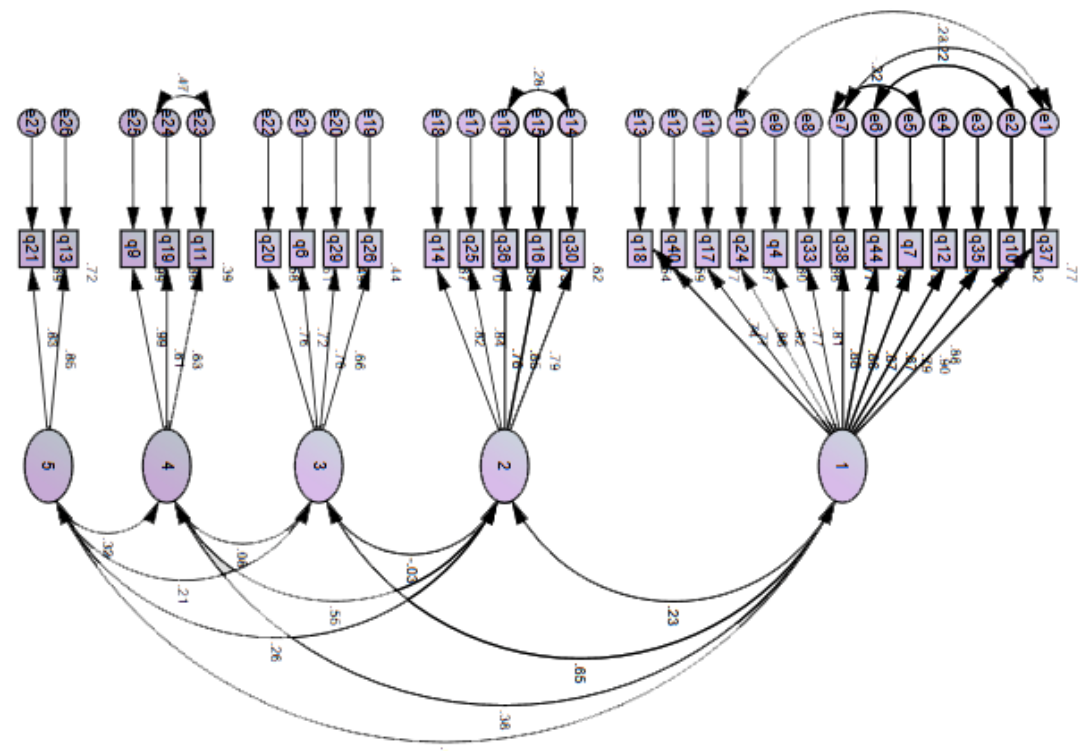

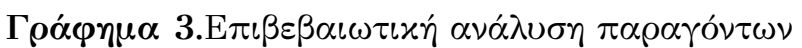

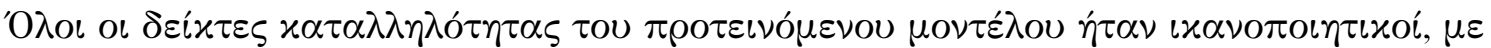

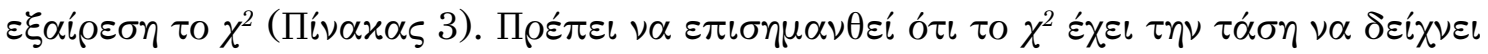

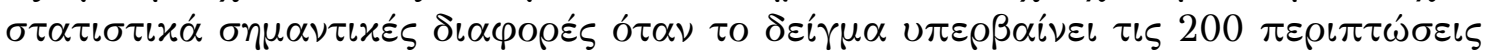

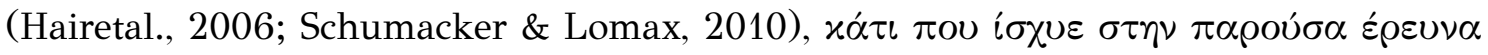
$(N=300)$

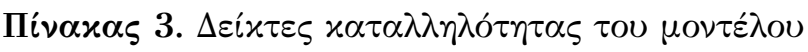

\begin{tabular}{|c|c|c|c|}
\hline$\Delta \varepsilon i x \tau \eta S$ & 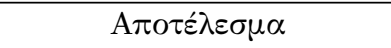 & $\Sigma v \nu \iota \sigma \tau \omega \dot{\mu} \mu \varepsilon \nu \eta \tau \iota \mu \eta^{\prime}$ & 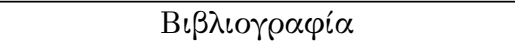 \\
\hline$\chi^{2}$ & $\chi^{2}(308)=575,16, p<0,001$ & $\mu \sigma \sigma \tau o p<0,05$ & Schumacker \& Lomax, 2010 \\
\hline$\chi^{2} / \mathrm{df}$ & 1,87 & $1-3$ & Kline, 2005 \\
\hline SRMR & 0,050 & $\leq 0,05$ & Klem, 2000; McDonald \& Ho, 2002 \\
\hline TLI & 0,950 & $\geq 0,95$ & Hu \& Bentler, 1999 \\
\hline NFI & 0,910 & $\geq 0,90$ & Bentler \& Bonett, 1980 \\
\hline RMSEA & 0,05 & $\leq 0,05$ & McDonald \& Ho, 2002 \\
\hline CFI & 0,956 & $\geq 0,95$ & Hu \& Bentler, 1999 \\
\hline
\end{tabular}

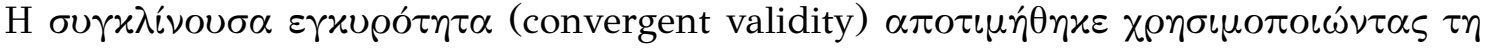

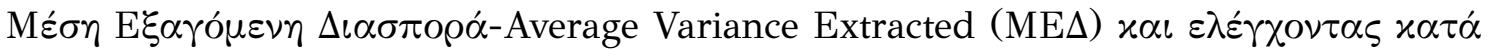

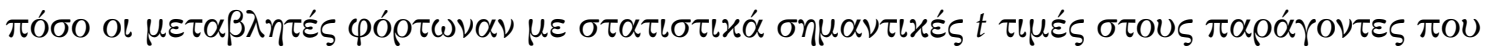

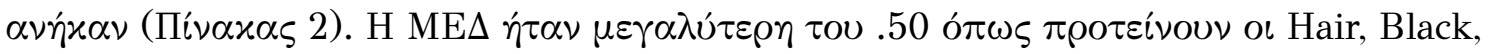

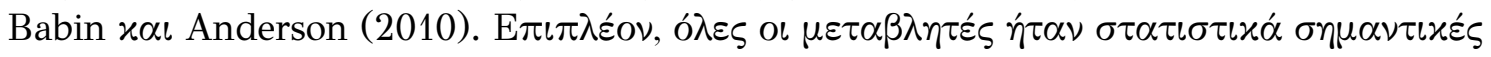

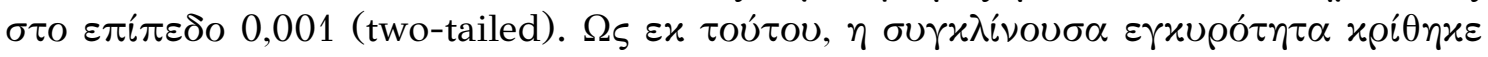

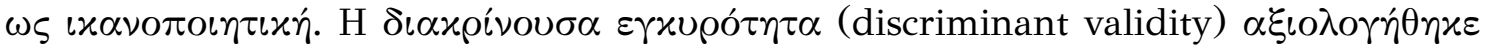

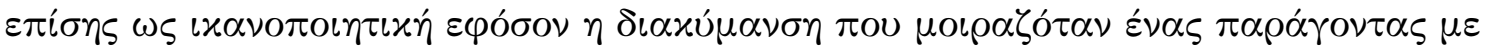




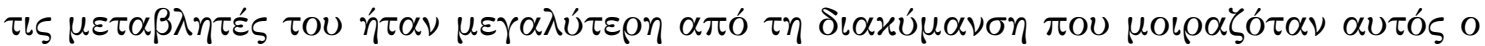

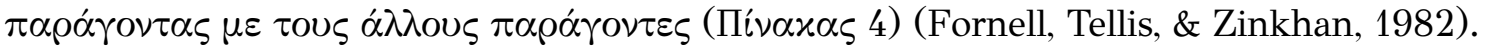

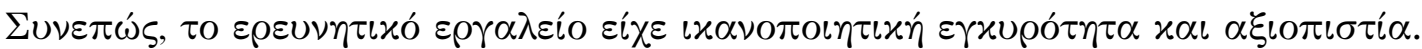

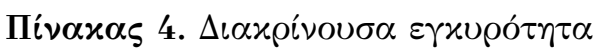

\begin{tabular}{|c|c|c|c|c|c|c|}
\hline \multirow[b]{2}{*}{ 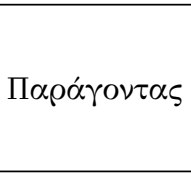 } & \multirow[b]{2}{*}{ KA } & \multicolumn{5}{|c|}{ ME $\Delta$} \\
\hline & & $\begin{array}{c}1 \\
B \varepsilon \lambda \tau i \omega \sigma \eta \\
\Theta E\end{array}$ & $\begin{array}{c}2 \\
\Theta \rho \eta \sigma x \varepsilon v \tau \iota x o ́ \tau \eta \tau \alpha\end{array}$ & $\begin{array}{c}3 \\
\mathrm{X} \rho \eta \sigma u \sigma^{\prime} \tau \tau \tau \alpha \\
\mathrm{M} \tau \Theta\end{array}$ & 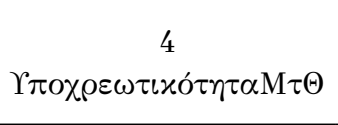 & 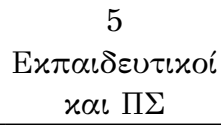 \\
\hline 1 & 0,964 & $(0,820)$ & & & & \\
\hline 2 & 0,907 & 0,231 & $(0,813)$ & & & \\
\hline 3 & 0,804 & 0,652 & $-0,035$ & $(0,712)$ & & \\
\hline 4 & 0,801 & 0,383 & 0,554 & 0,079 & $(0,765)$ & \\
\hline 5 & 0,826 & 0,595 & 0,258 & 0,205 & 0,324 & $(0,839)$ \\
\hline
\end{tabular}

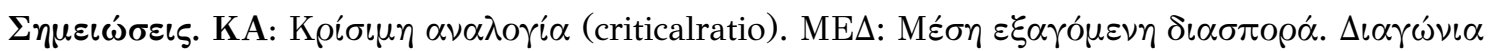

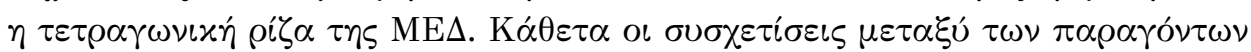

\section{$\Delta o \mu \iota \varkappa \alpha ́ \alpha$}

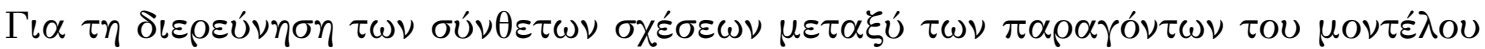

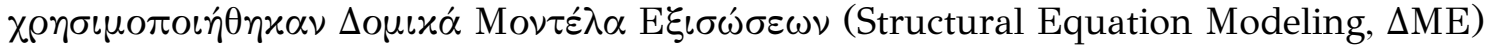

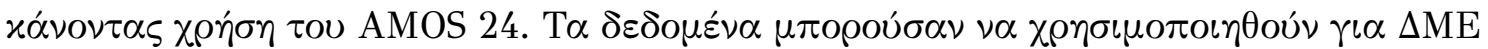

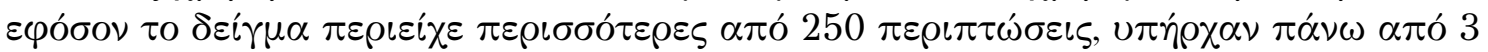

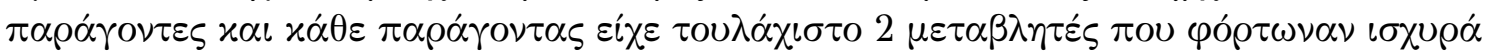

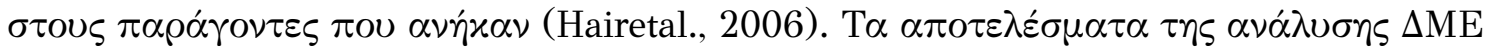

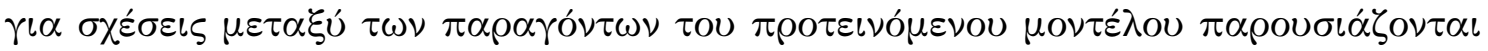

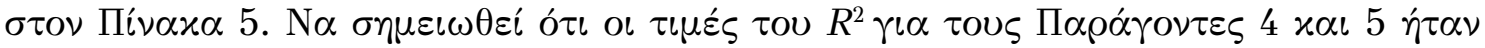

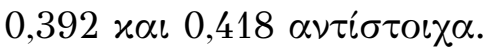

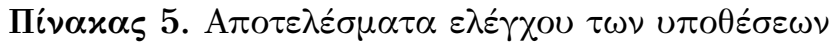

\begin{tabular}{|c|c|c|c|c|c|}
\hline$\Upsilon \pi \delta ́ \theta \varepsilon \sigma \eta$ & $\Delta \iota \alpha \delta \rho о \mu \dot{n}$ & $\begin{array}{c}\Sigma v \nu \tau \varepsilon \lambda \varepsilon \sigma \tau \eta^{\prime} \varsigma \\
\delta \iota \alpha \delta \rho O \mu \eta \dot{s}(\beta)\end{array}$ & $t$-value & $P$ & 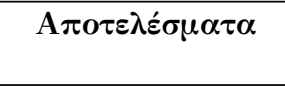 \\
\hline $\mathrm{Y} 1 \alpha$ & $1 \rightarrow 4$ & 0,270 & 3,361 & $<0,001$ & $\Upsilon \pi 0 \sigma \tau \eta \rho i \zeta \varepsilon \tau \alpha \iota$ \\
\hline $\mathrm{Y} 1 \beta$ & $1 \rightarrow 5$ & 0,810 & 8,346 & $<0,001$ & $\Upsilon \pi 0 \sigma \tau \eta \rho i \zeta \varepsilon \tau \alpha \iota$ \\
\hline$\Upsilon 2 \alpha$ & $2 \rightarrow 4$ & 0,506 & 6,027 & $<0,001$ & $\Upsilon \pi 0 \sigma \tau \eta \rho i \zeta \varepsilon \tau \alpha \iota$ \\
\hline $\mathrm{Y} 2 \beta$ & $2 \rightarrow 5$ & 0,069 & 1,137 & 0,256 & $\Delta \varepsilon \nu$ v $\pi 0 \sigma \tau \eta \rho i \zeta \varepsilon \tau \alpha l$ \\
\hline $\mathrm{Y} 3 \alpha$ & $3 \rightarrow 4$ & $-0,142$ & $-1,669$ & 0,095 & $\Delta \varepsilon \nu$ v \\
\hline $\mathrm{Y} 3 \beta$ & $3 \rightarrow 5$ & $-0,327$ & $-3,254$ & 0,001 & $\Upsilon \pi 0 \sigma \tau \eta \rho i \zeta \varepsilon \tau \alpha \iota$ \\
\hline Y4 & $5 \rightarrow 4$ & 0,023 & 0,315 & 0,753 & 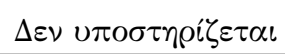 \\
\hline
\end{tabular}

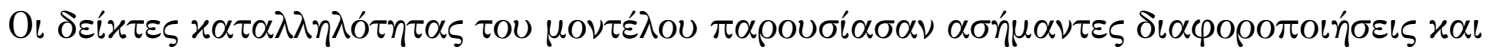

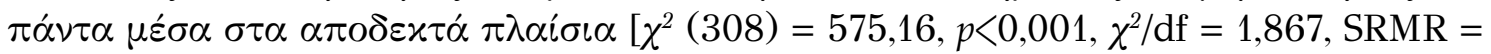

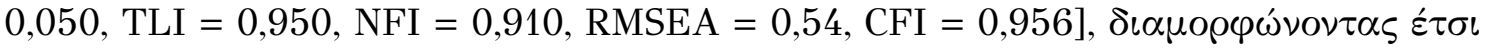

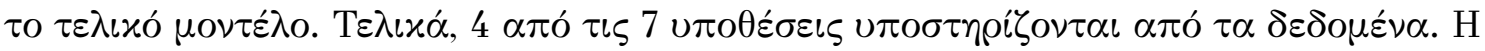




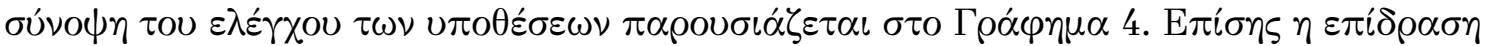

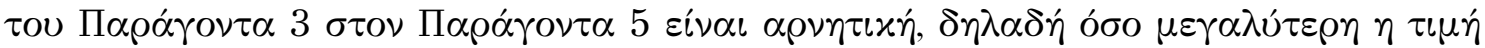

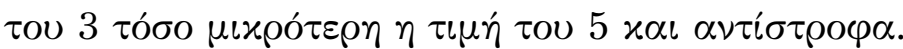

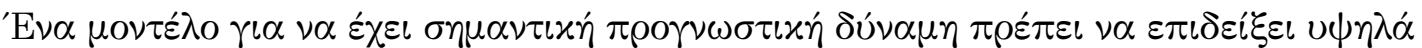

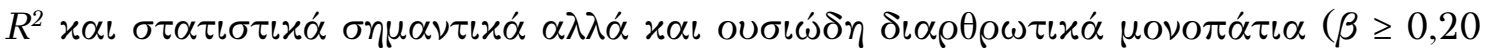

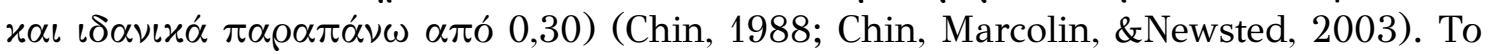

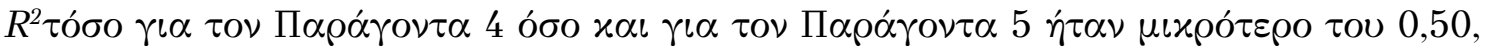

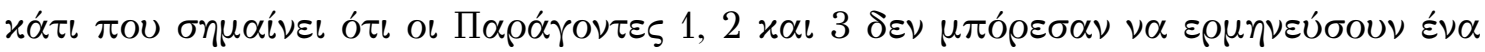

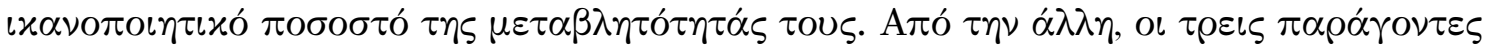

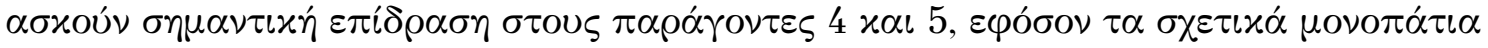

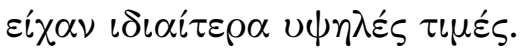

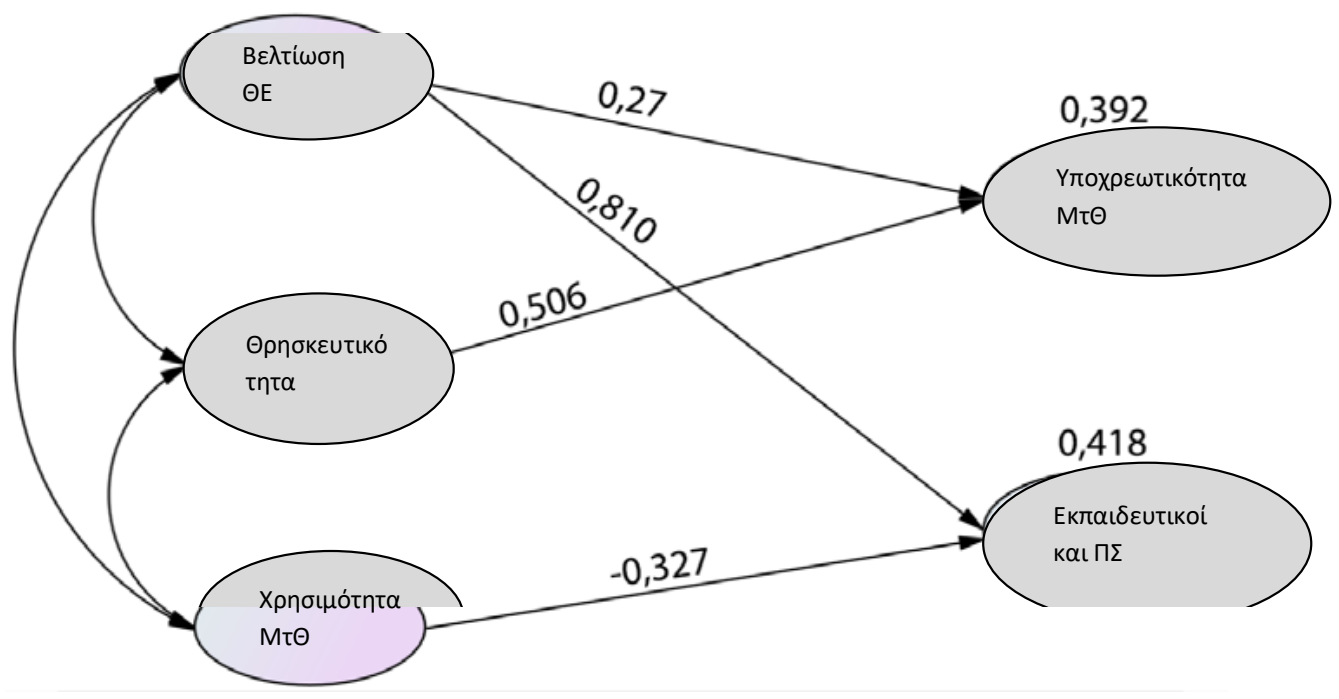

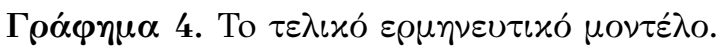

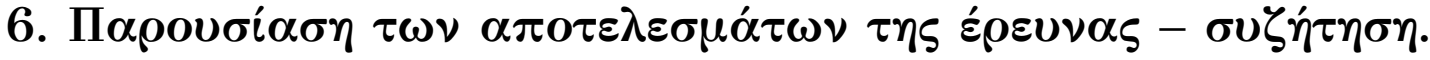

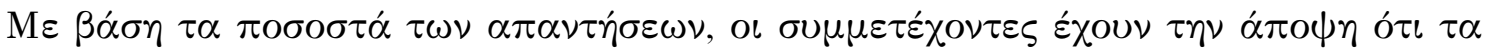

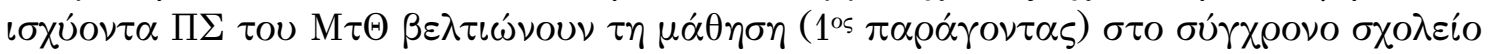

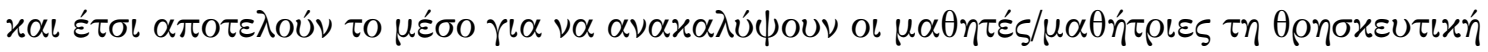

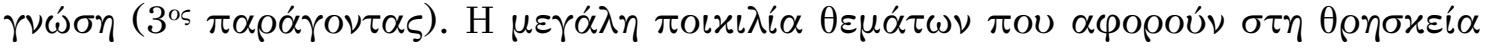

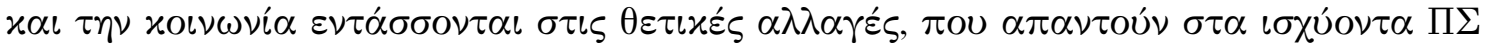

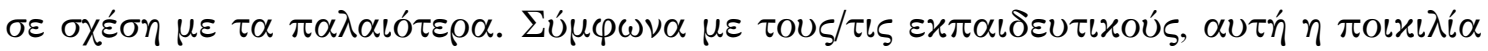

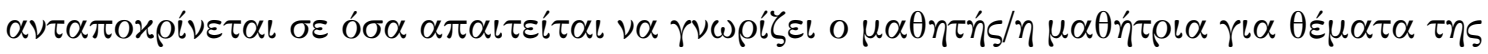

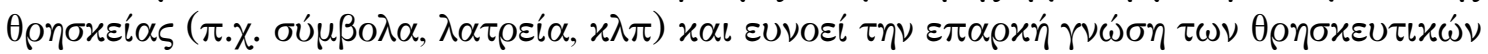

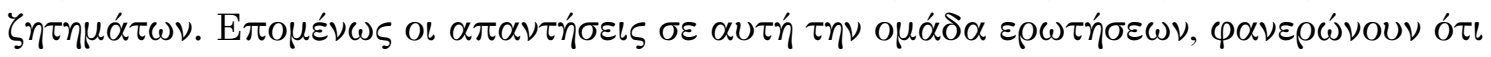

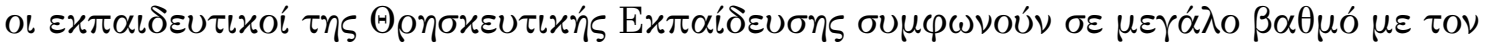

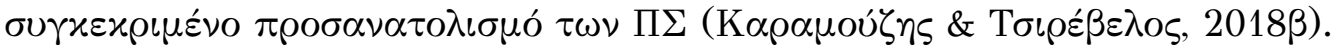

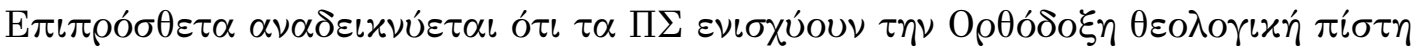




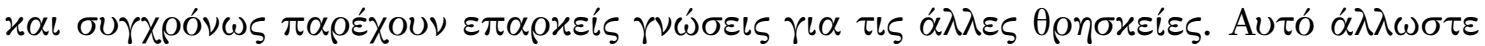

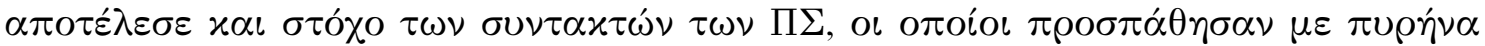

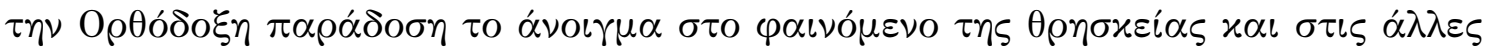

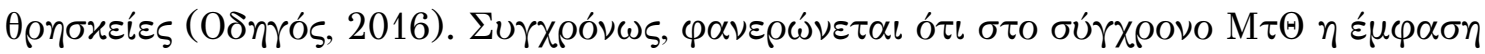

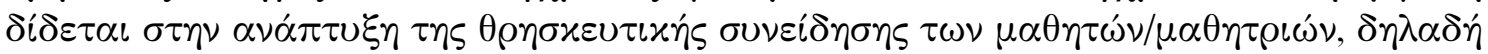

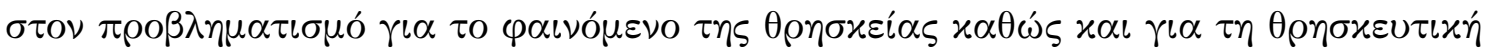

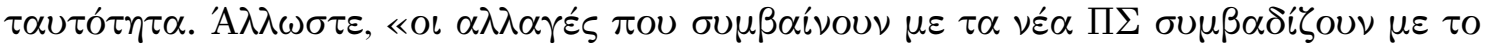

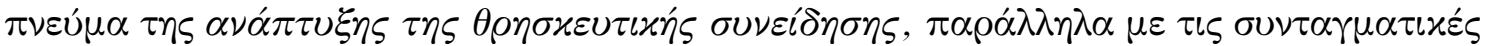

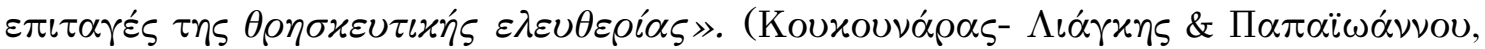
2016).

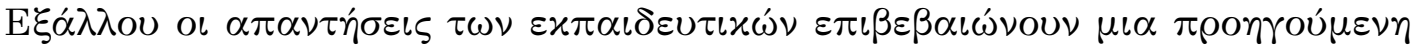

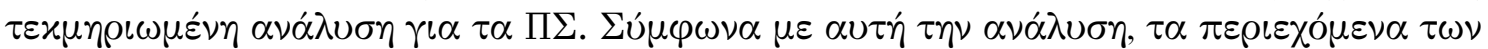

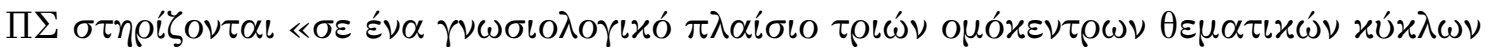

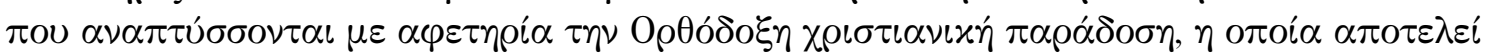

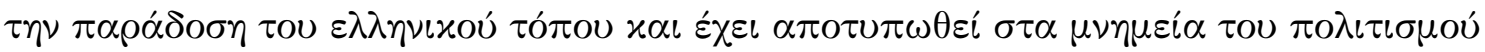

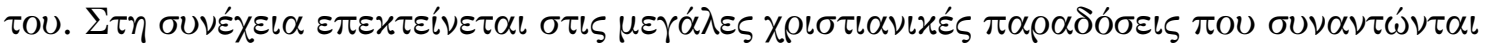

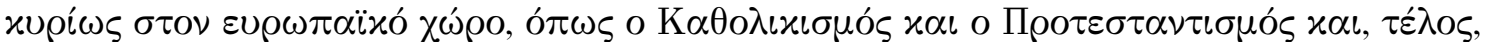

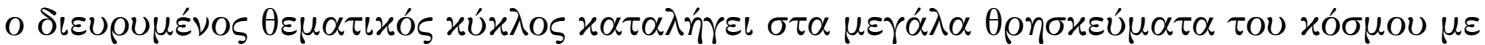

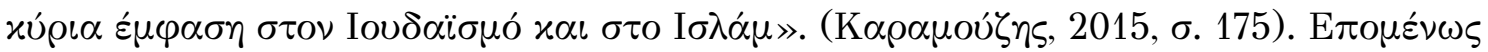

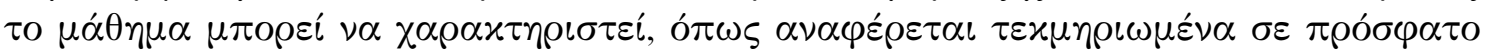

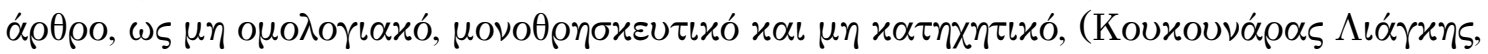

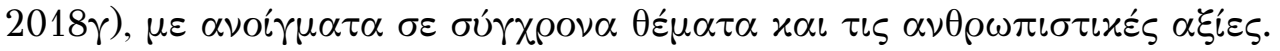

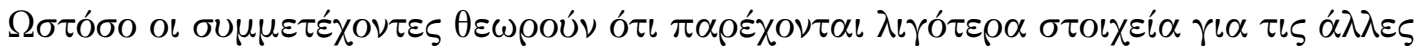

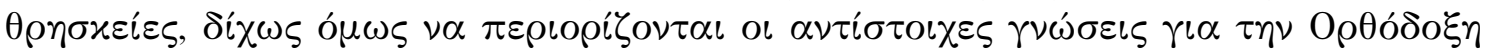

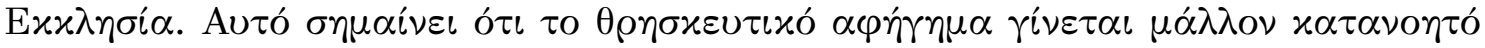

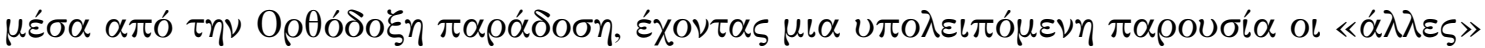

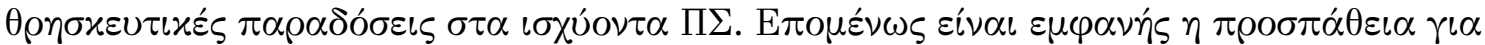

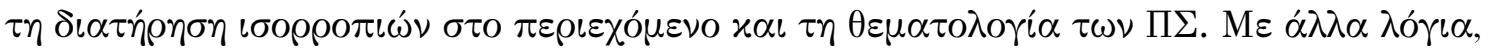

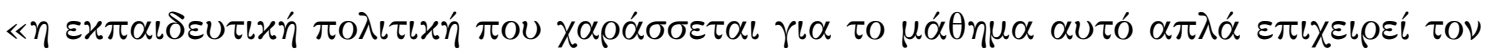

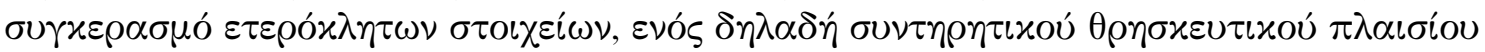

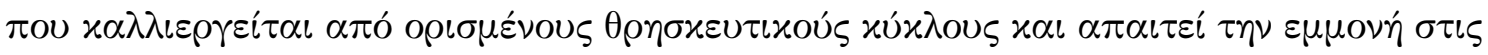

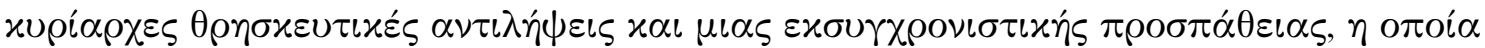

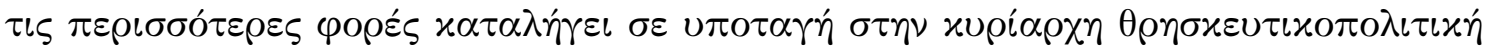
$\alpha \nu \tau i ́ \lambda \eta \psi \eta \gg(K \alpha \rho \alpha \mu o u ́ \zeta \eta \varsigma, 2015, \sigma .171)$.

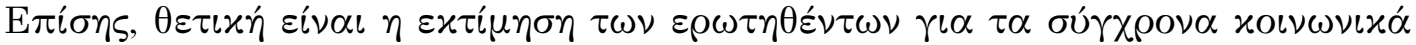

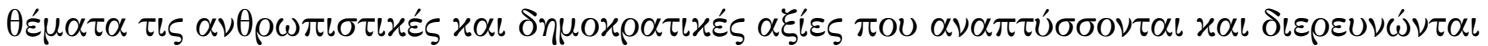

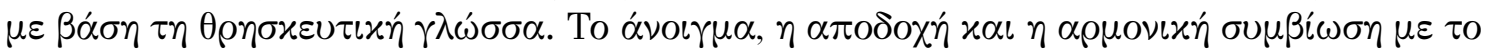

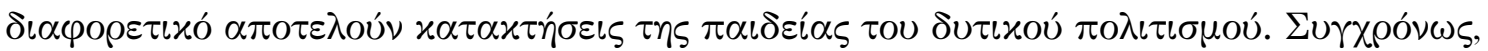

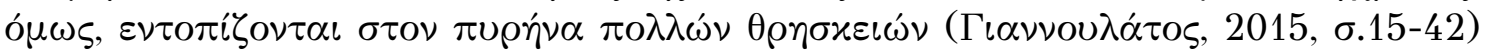

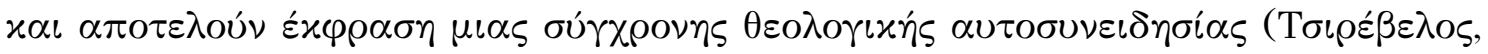

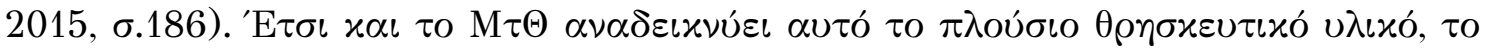

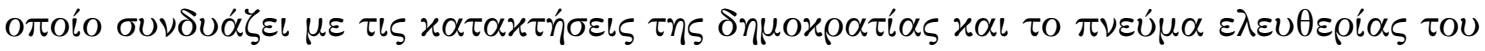

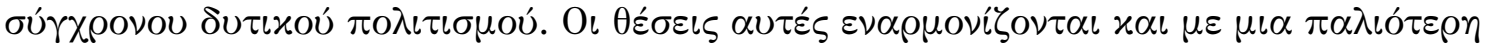

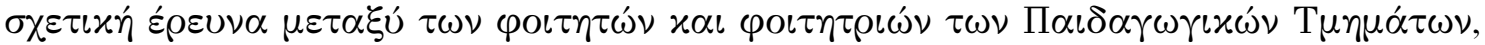




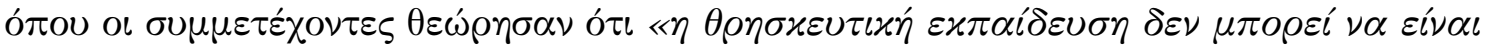

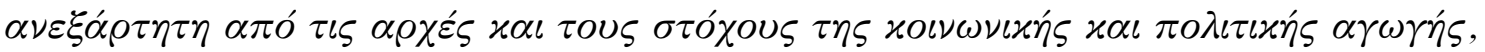

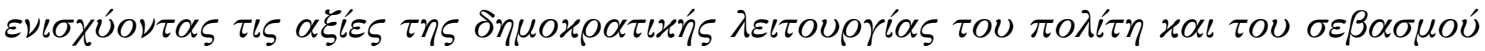

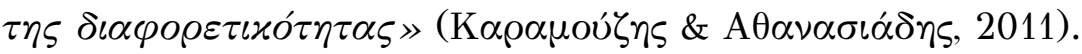

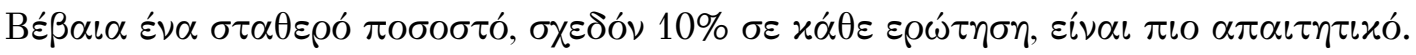

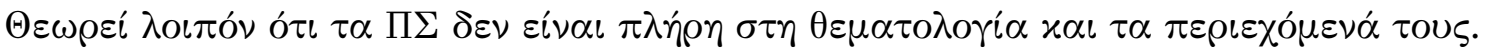

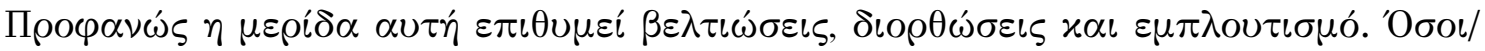

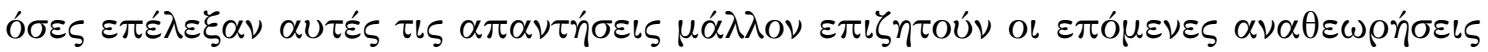

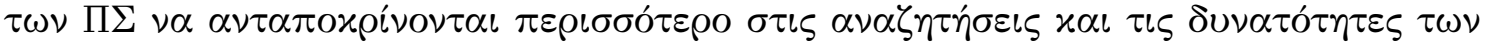

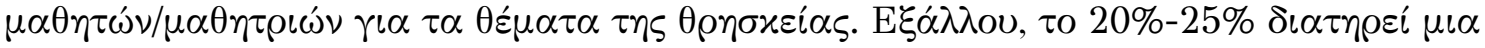

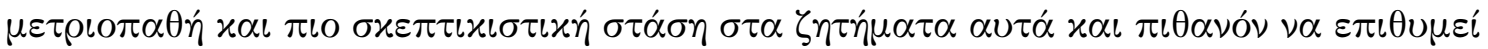

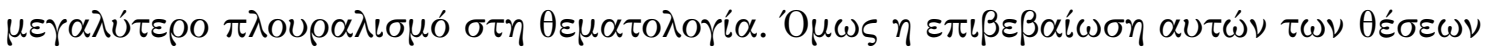

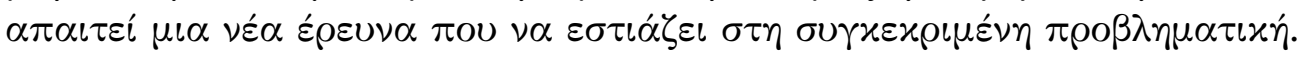

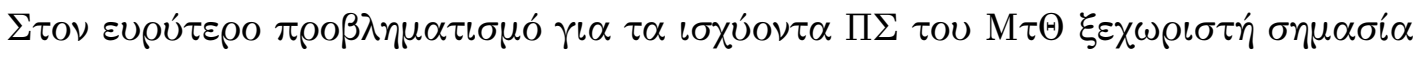

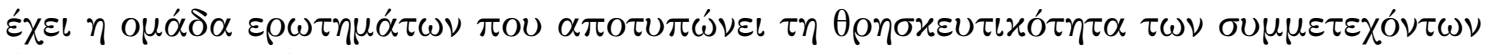

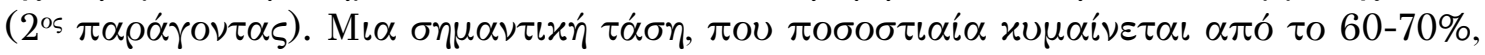

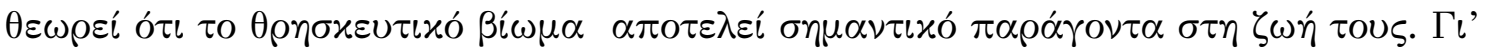

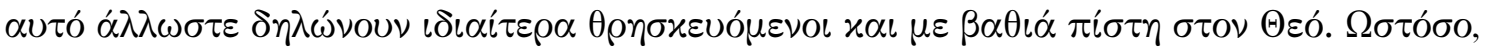

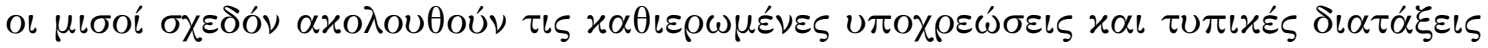

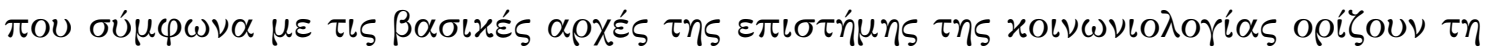

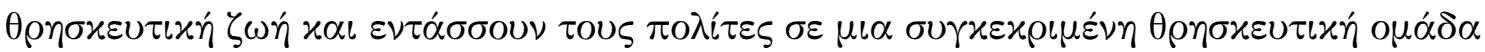

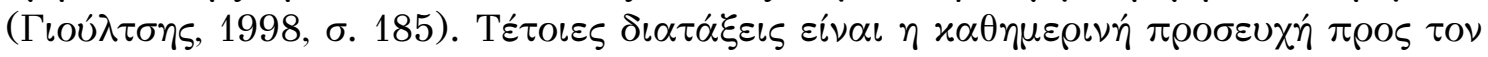

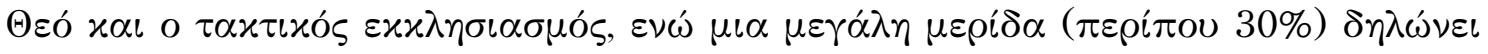

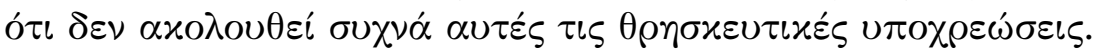

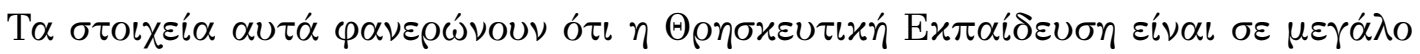

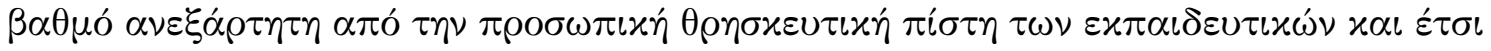

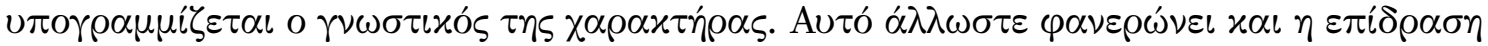

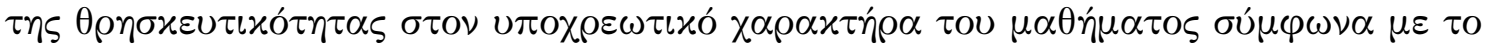

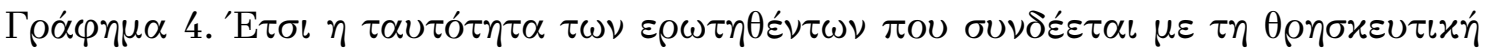

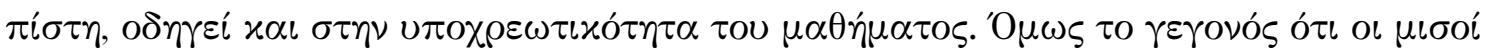

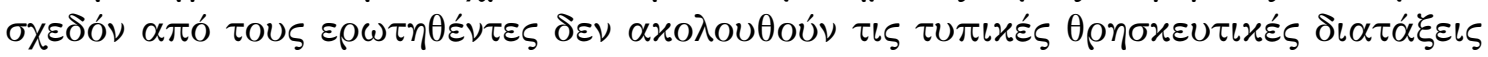

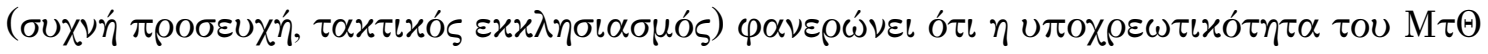

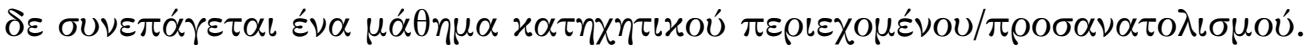

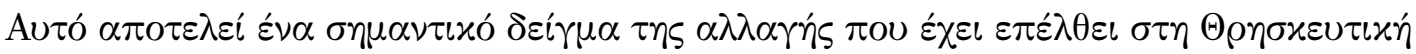

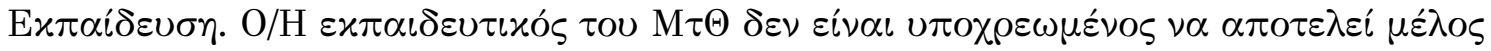

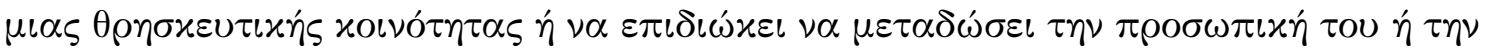

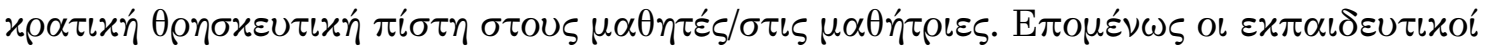

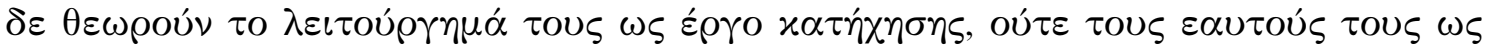

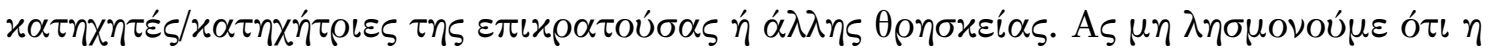

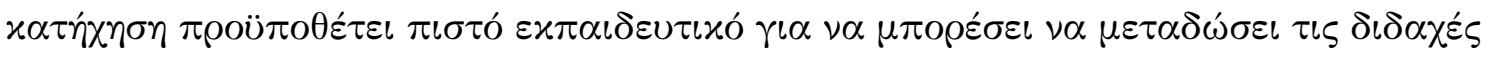

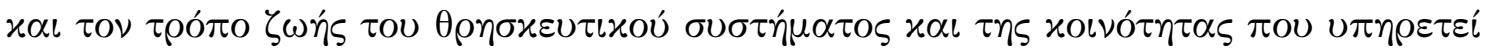

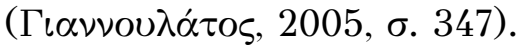

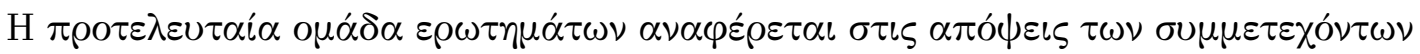




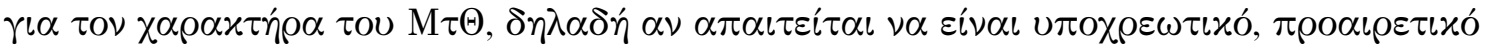

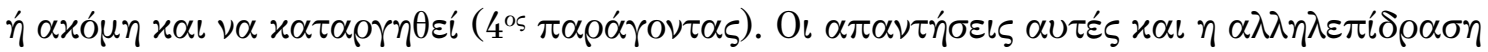

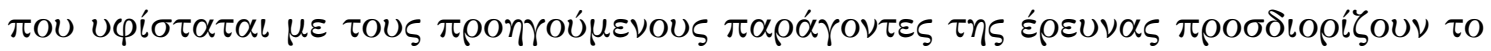

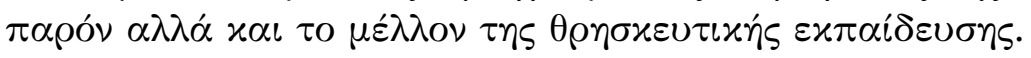

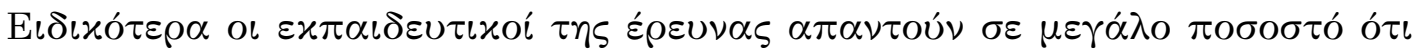

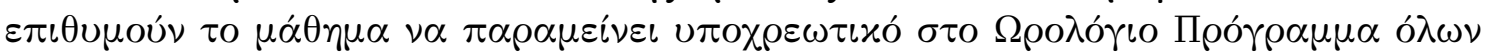

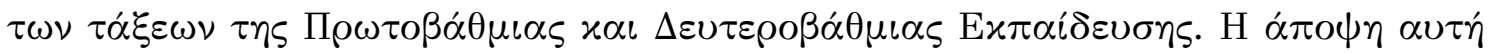

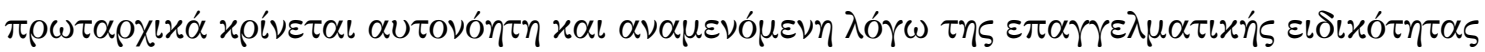

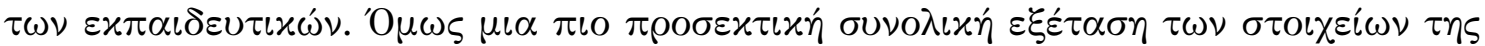

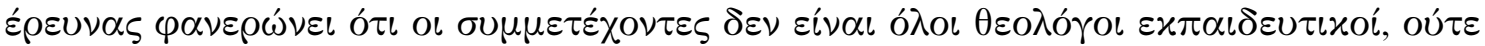

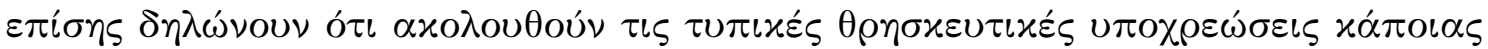

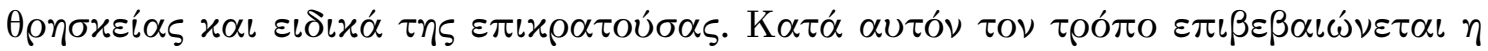

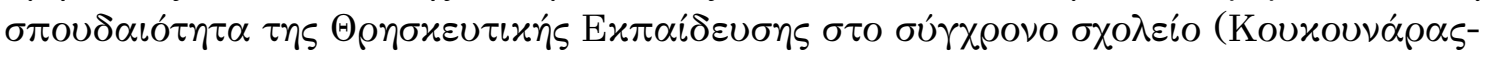

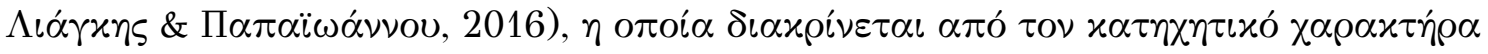

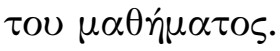

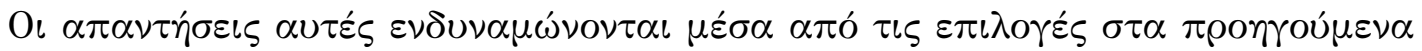

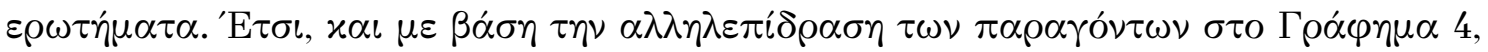

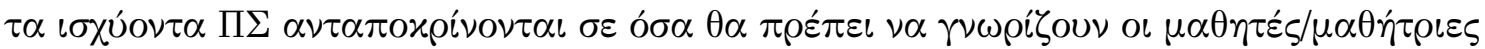

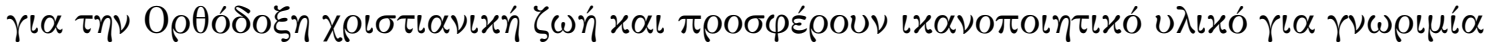

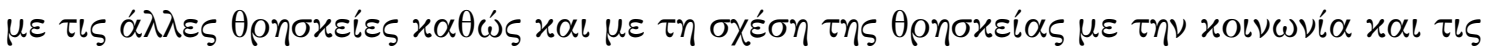

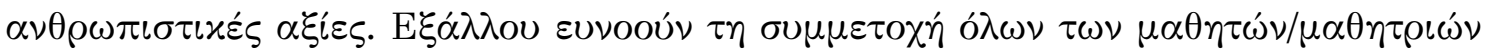

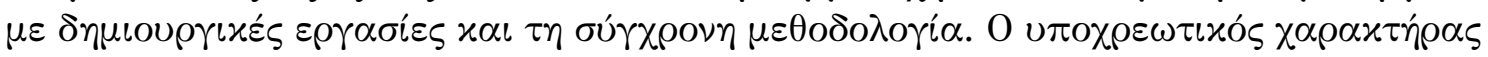

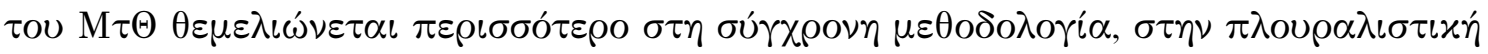

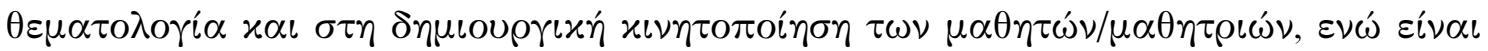

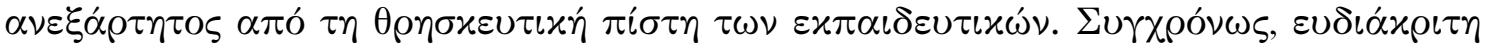

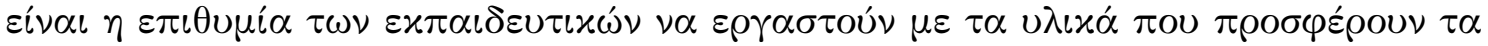

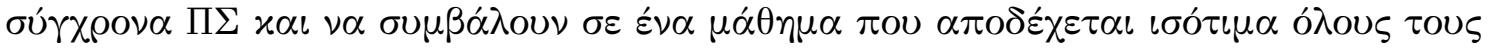

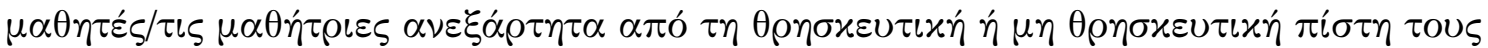

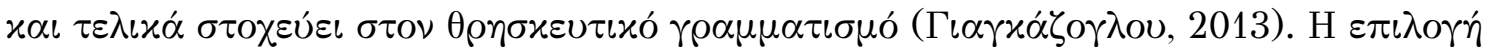

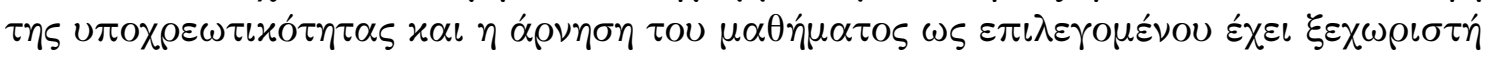

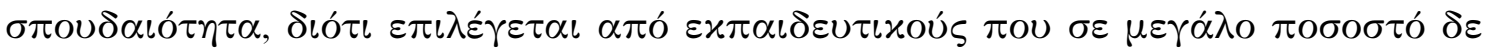

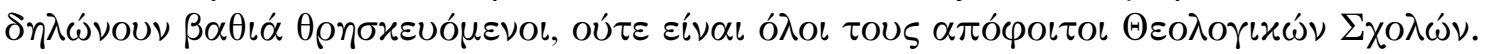

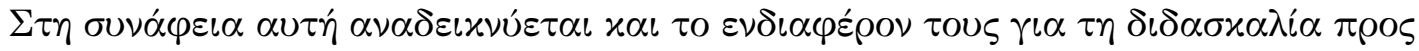

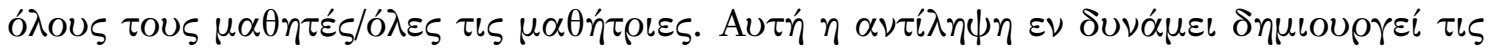

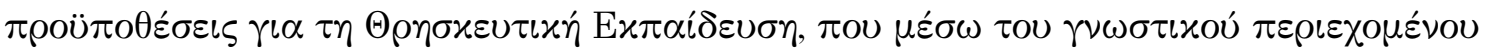

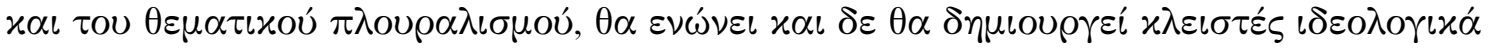

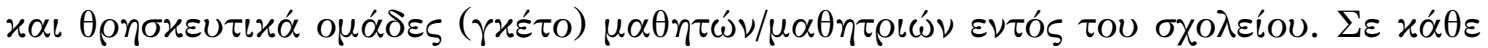

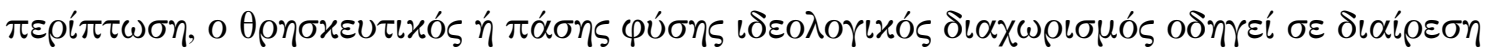

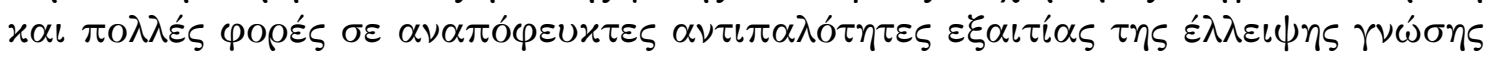

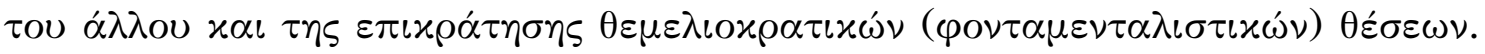

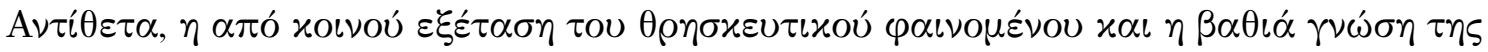

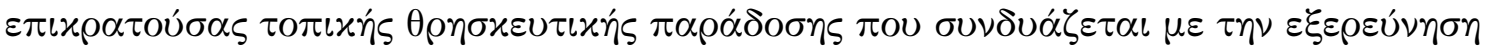

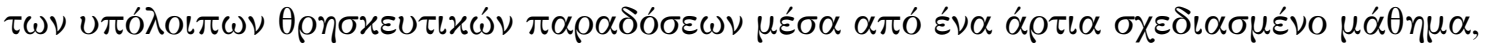

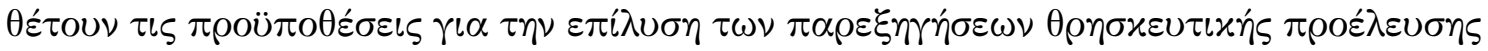




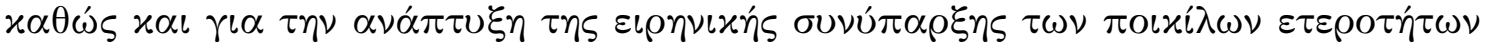

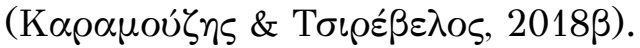

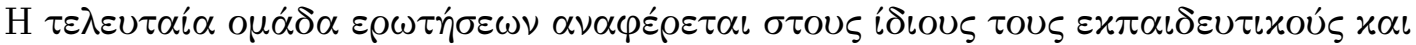

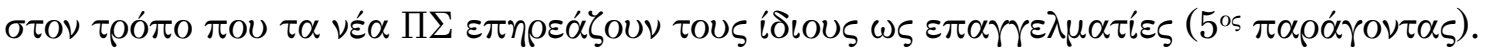

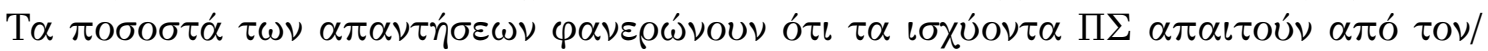

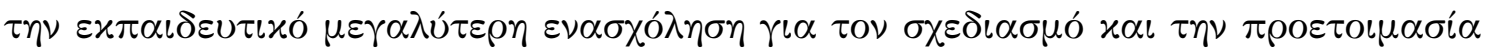

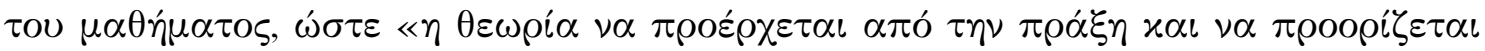

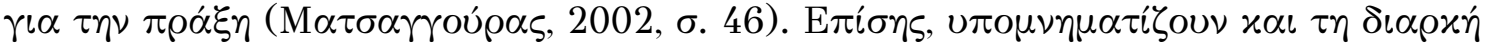

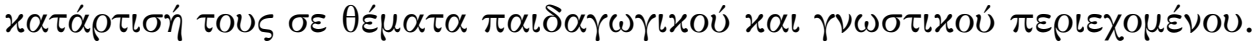

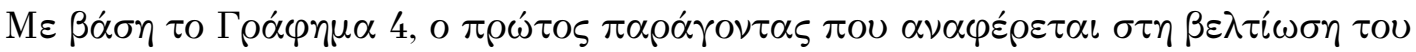

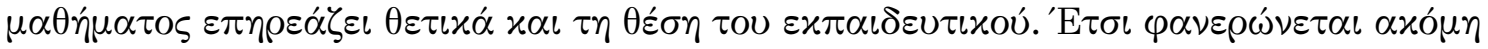

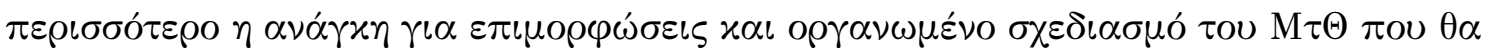

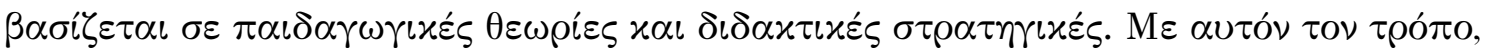

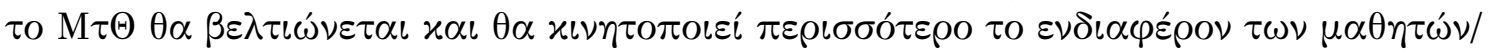

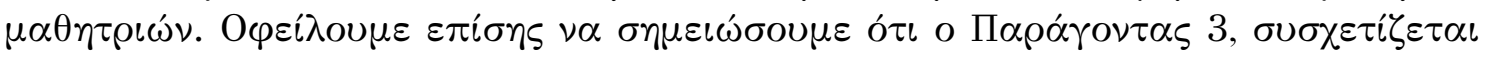

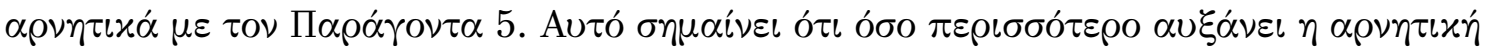

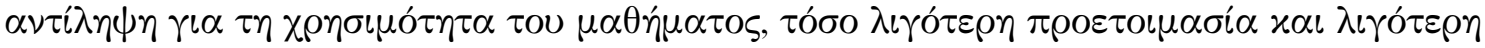

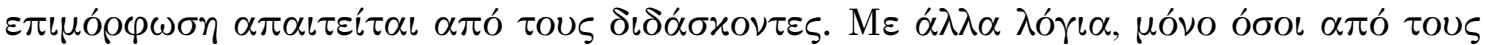

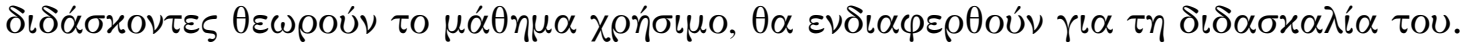

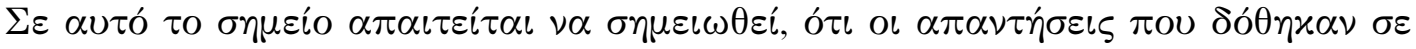

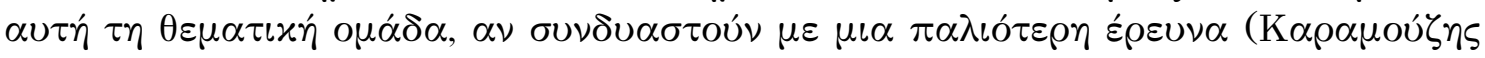

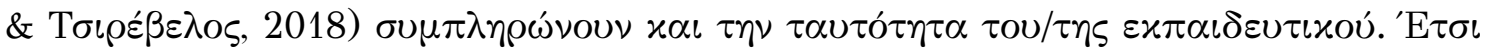

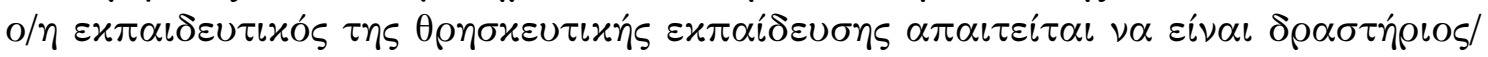

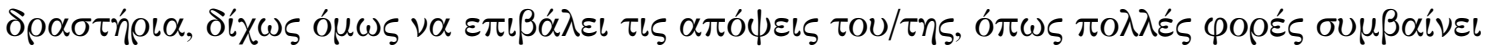

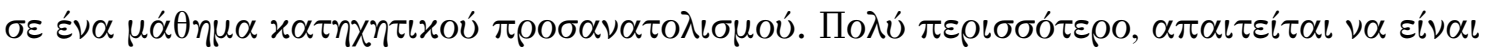

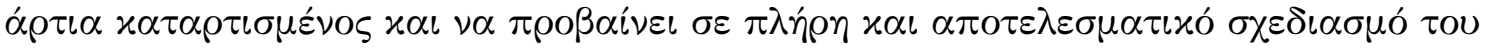

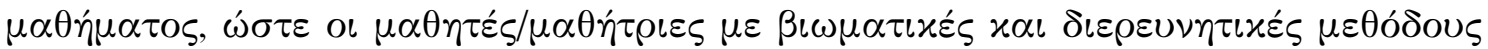

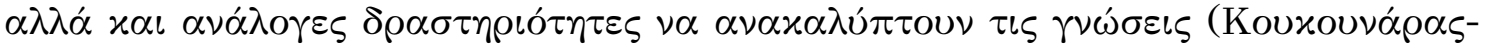
$\Lambda \iota \alpha ́ \gamma \times \eta \bar{c}, 2017)$.

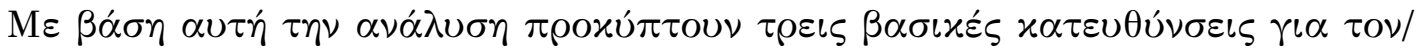

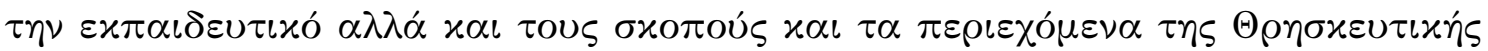

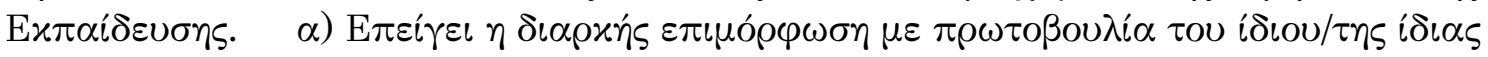

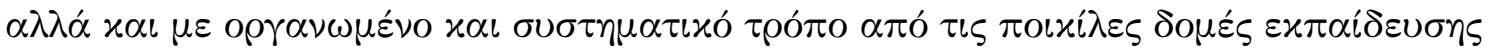

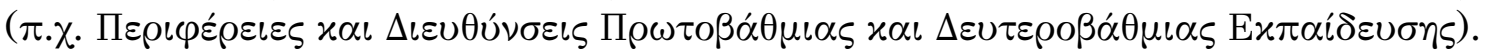

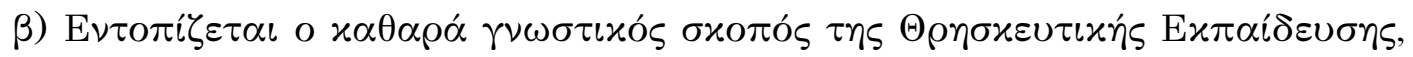

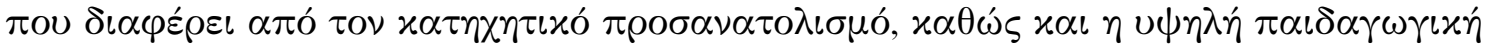
$\alpha \lambda \lambda \alpha \gamma \eta ́ \eta$

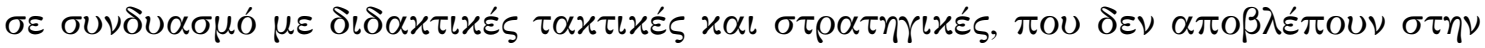

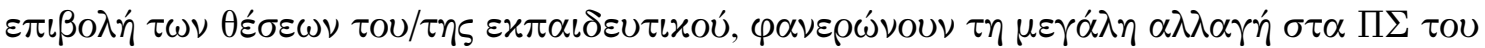

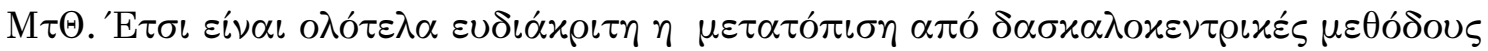

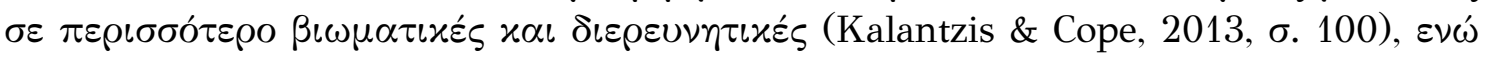

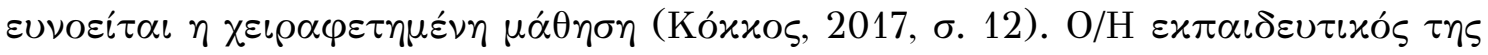

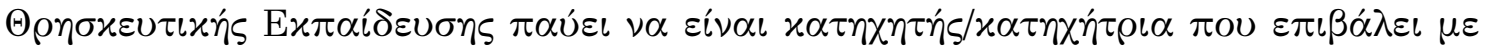




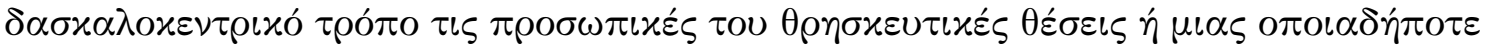

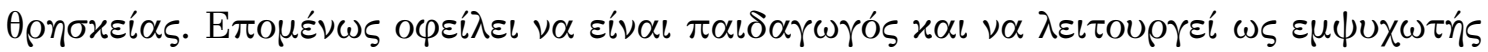

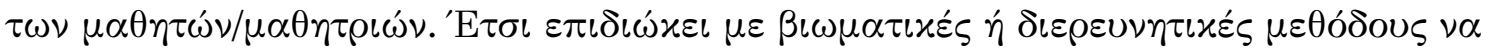

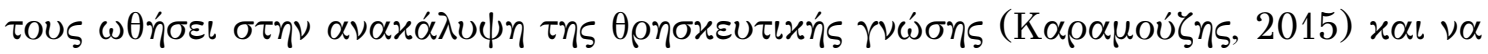

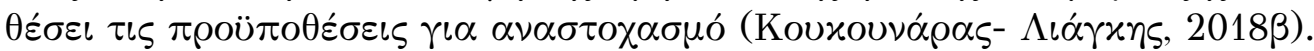

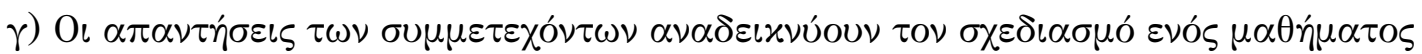

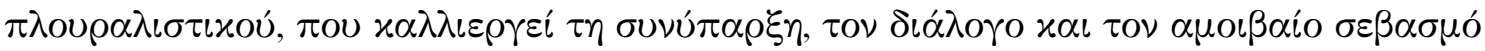

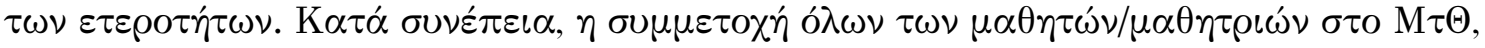

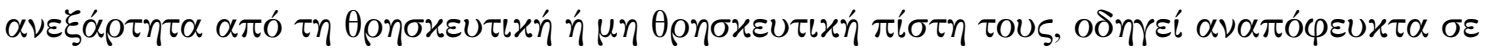

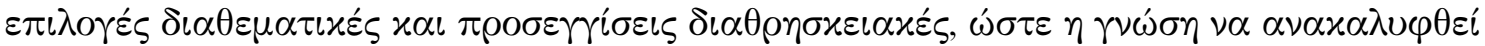

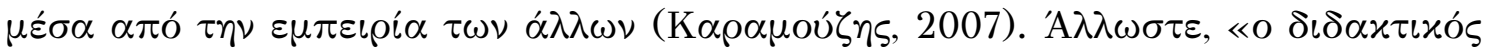

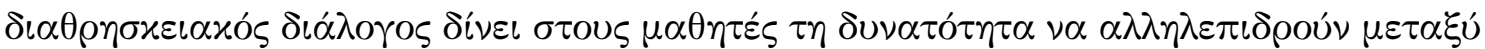

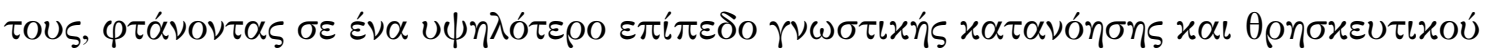

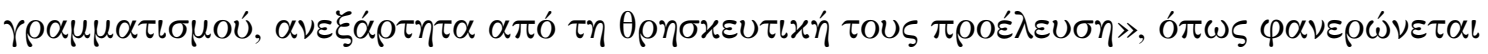

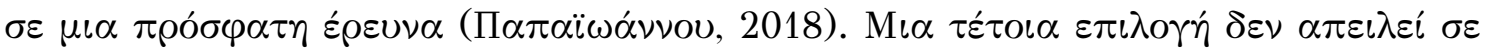

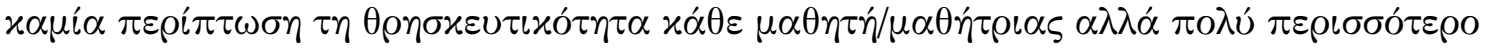

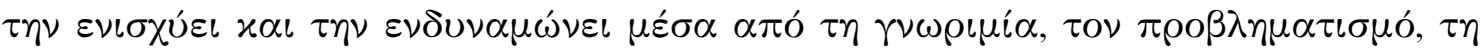

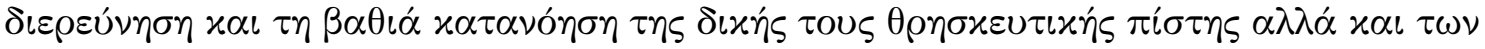

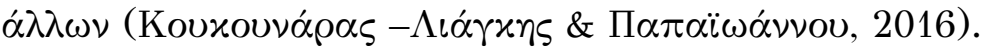

\section{$\Sigma v \mu \pi \varepsilon \rho \alpha ́ \alpha \mu \alpha \tau \alpha-\Pi \rho о \tau \alpha ́ \alpha \varepsilon \iota \varsigma$}

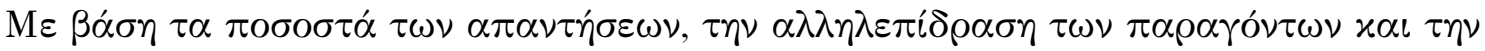

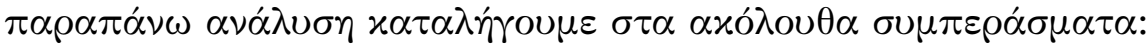

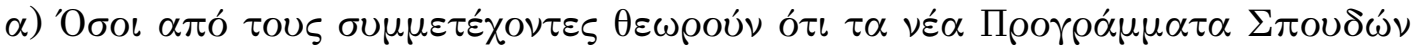

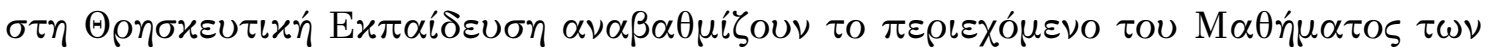

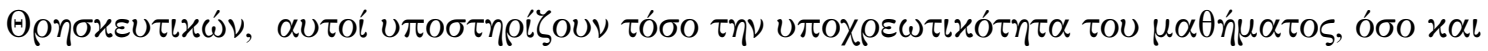

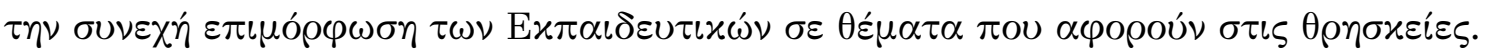

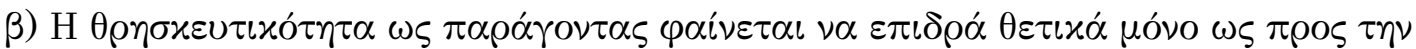

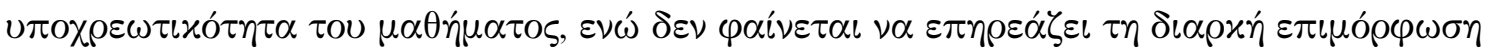

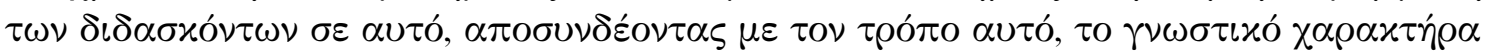

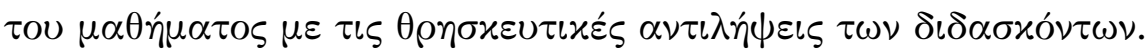

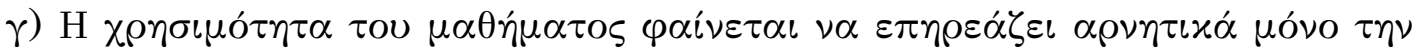

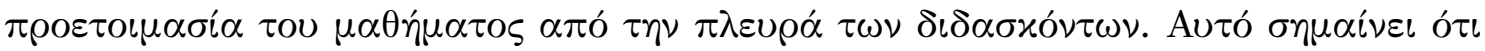

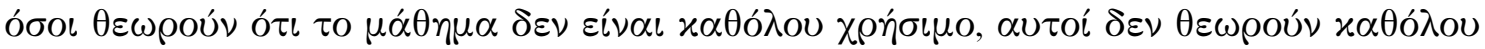

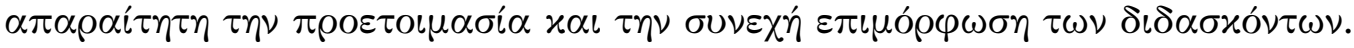

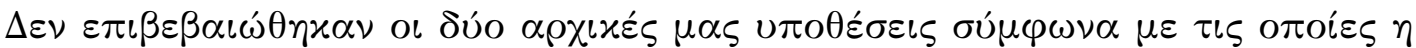

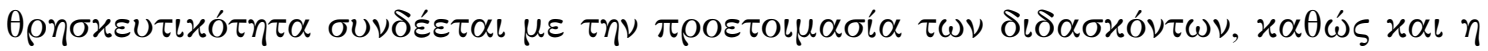

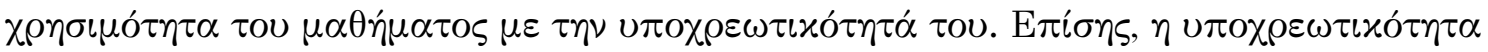

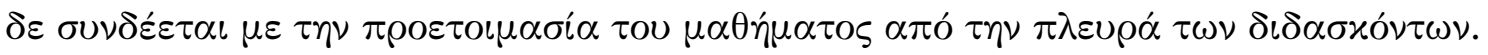

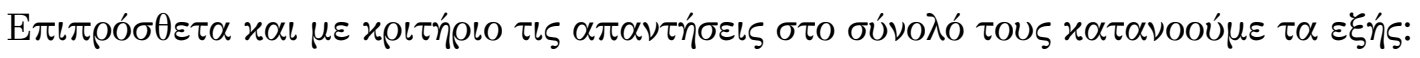

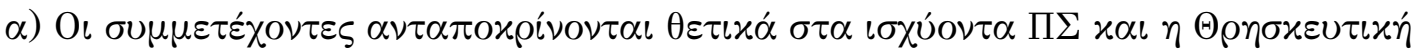

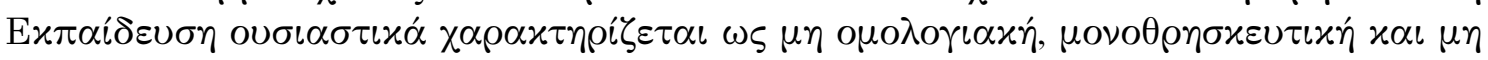




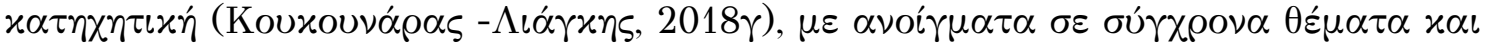
$\tau \iota \varsigma \alpha \nu \theta \rho \omega \pi \iota \sigma \tau \iota x \varepsilon ́ \varsigma$ $\alpha \xi i \varepsilon \varsigma$.

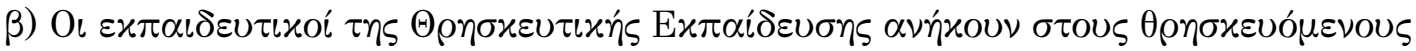

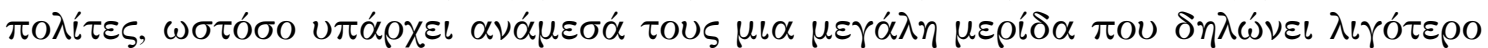

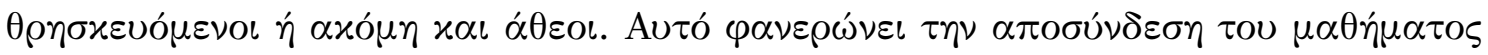

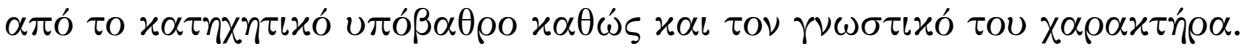

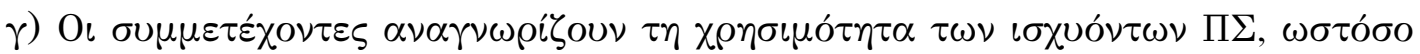

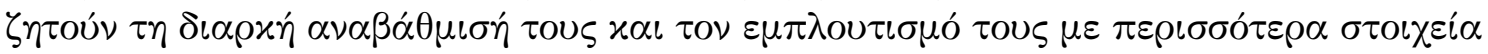

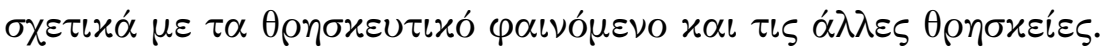

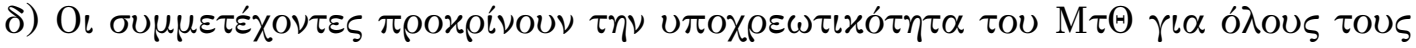

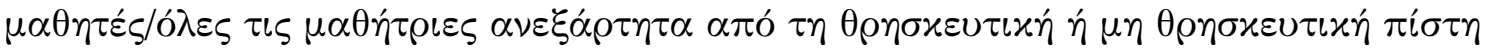

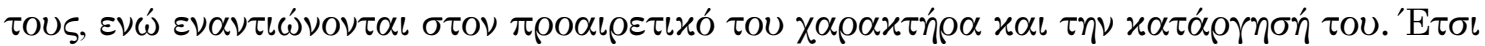

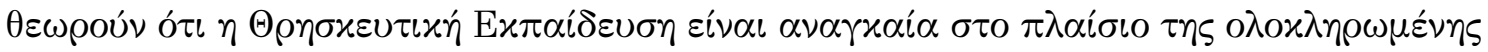

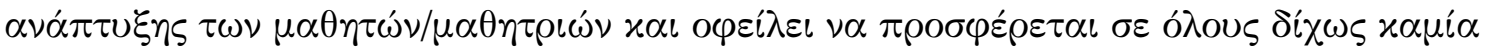

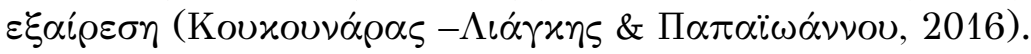

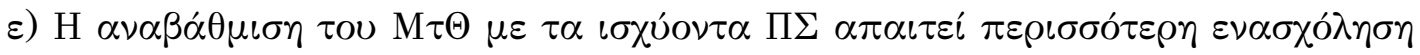

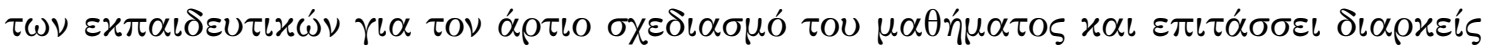

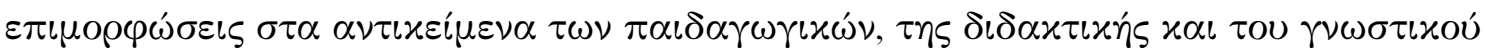
$\pi \varepsilon \delta i ́ o v$.

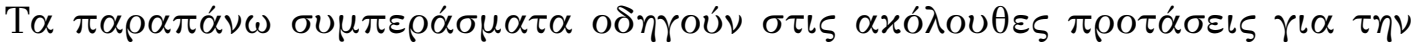

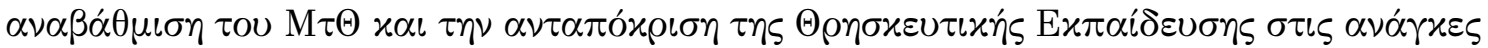

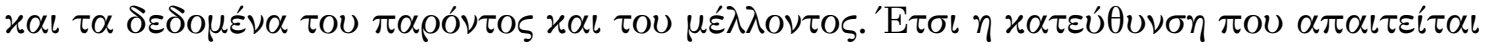

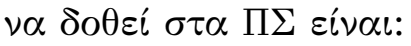

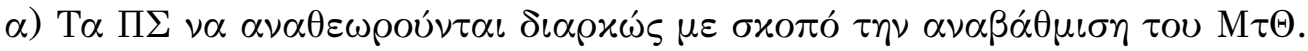

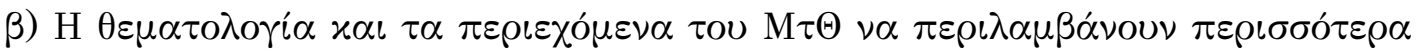

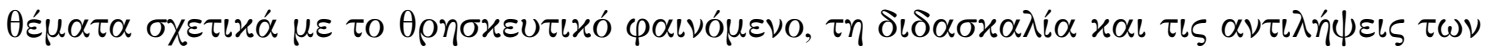

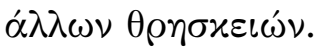

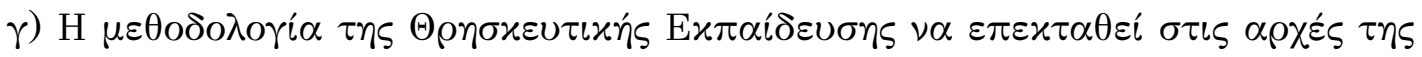

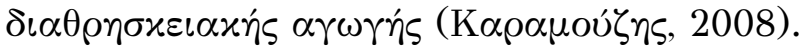

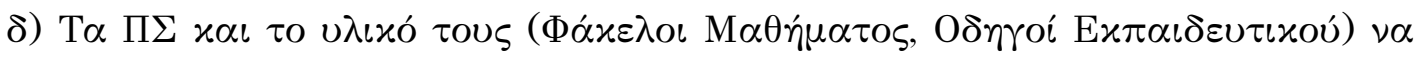

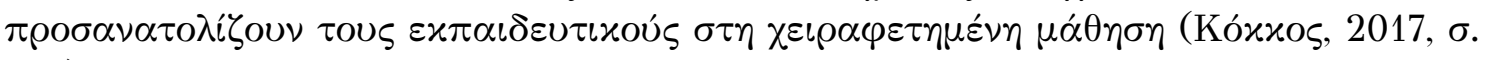

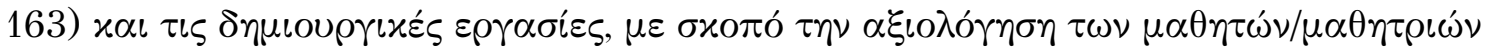

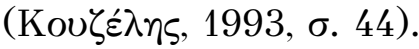

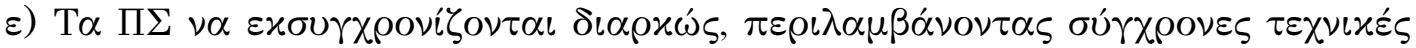

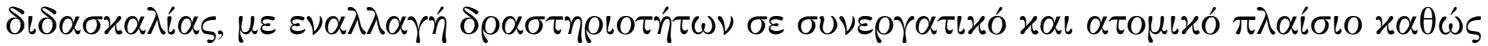

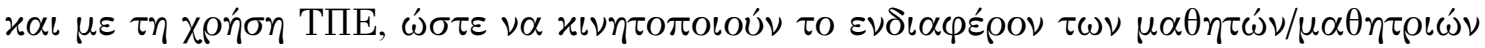

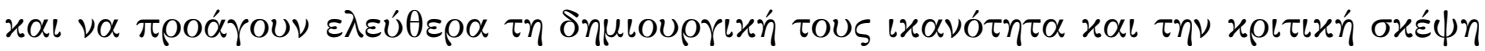

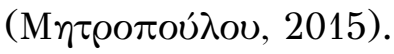

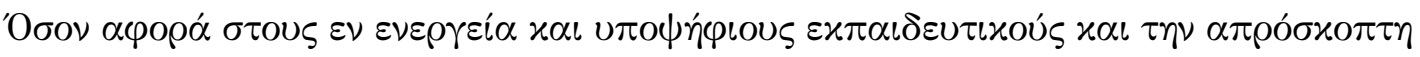

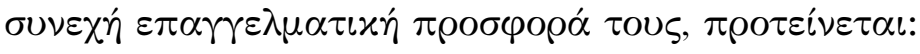




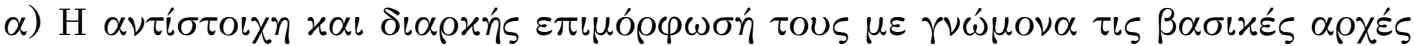

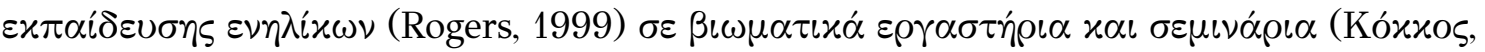

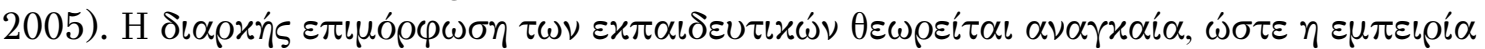

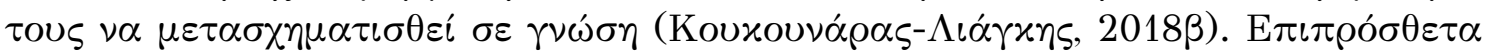

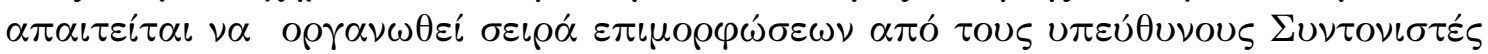

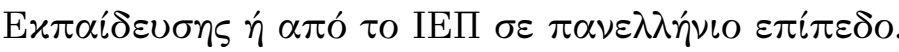

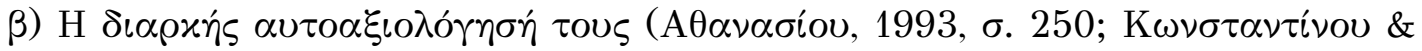

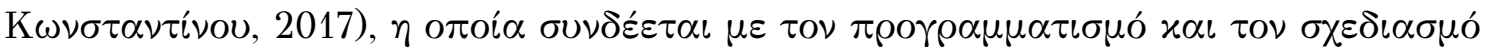

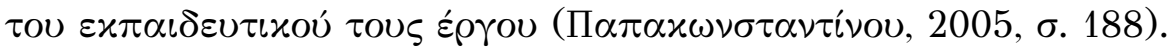

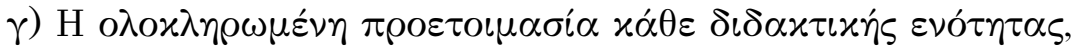

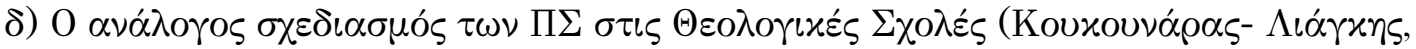

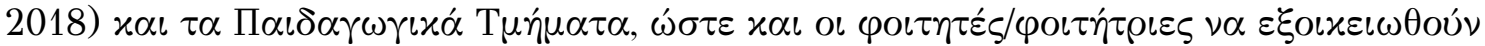

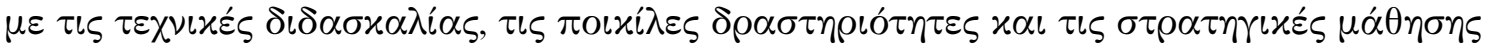

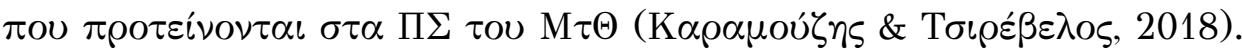

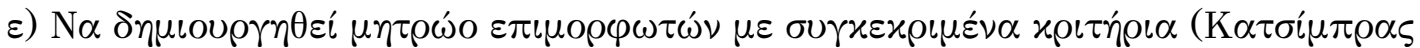

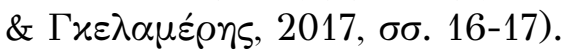

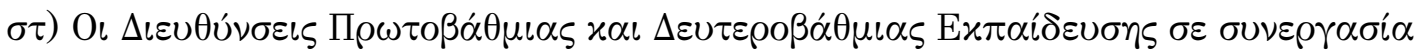

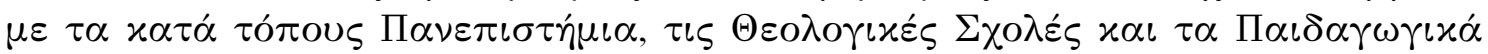

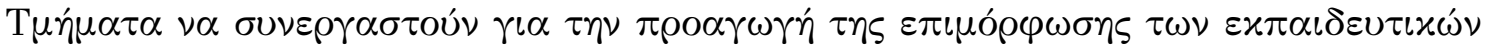

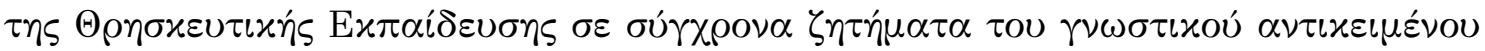

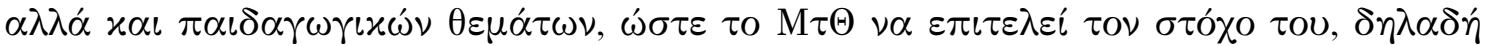

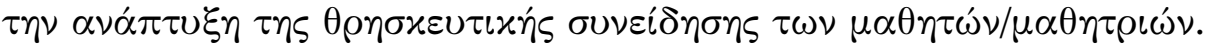

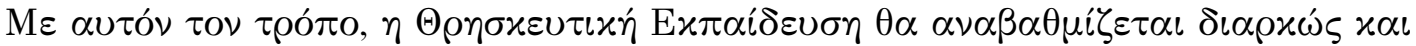

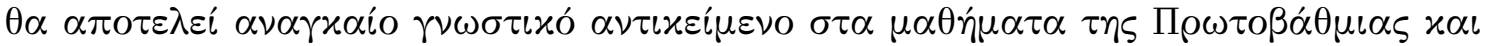

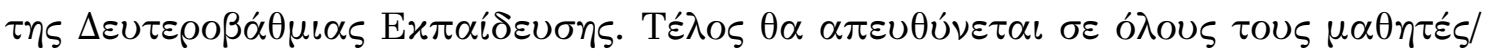

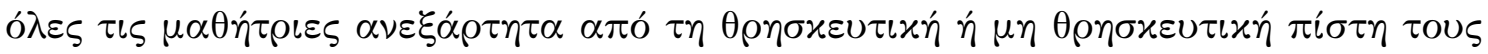

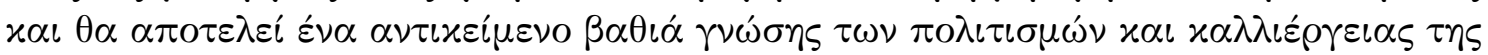

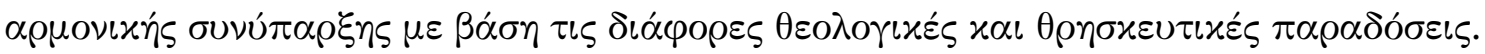

\section{Bı $\beta \lambda \iota \gamma \gamma \rho \alpha \varphi i \alpha$}

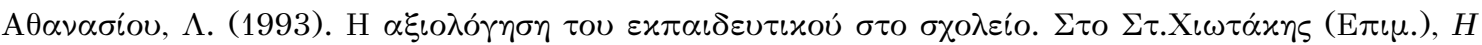

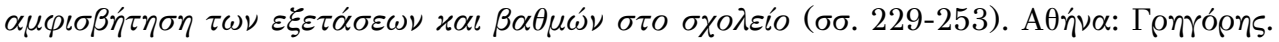

Bentler, P. M., \& Bonett, D. G. (1980). Significance tests and goodness of fit in the analysis of covariance structures. Psychological Bulletin, 88 (3), 588.

Cattel, R.B. (1978). The scientific use of factor analysis. New York: Plenum.

Chin, W. W. (1988). Issues and opinion on structural equation modeling. MIS Quarterly, 22, vii-xvi.

Chin, W. W., Marcolin, B. L., \& Newsted, P. R. (2003). A partial least squares latent variable modeling approach for measuring interaction effects: Results from a Monte Carlo simulation study and an electronic-mail emotion/adoption study. Information Systems Research, 14(2), 189-217.

Cohen, E. (1994). Designing groupworks. Strategies for heterogeneous classroom. NewYork: Teachers, College Press. 


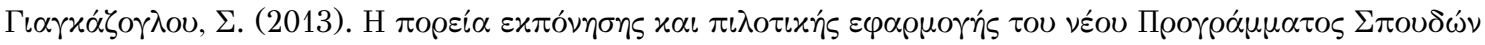

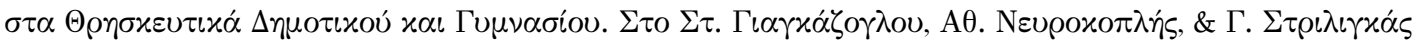

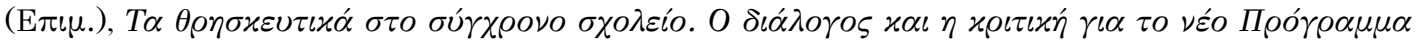

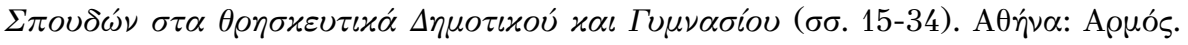

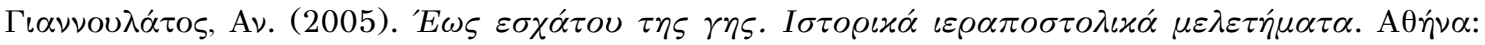
A $\pi 0 \sigma \tau 0 \lambda \iota x \dot{n} \Delta \iota \alpha x o v i \alpha$.

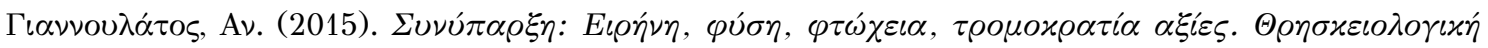

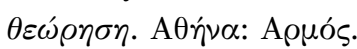

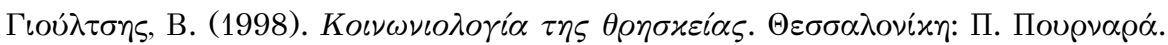

DeVellis, R. F. (2003). Scale development: Theory and applications (2nd ed.). Newbury Park, CA: SAGE Publications.

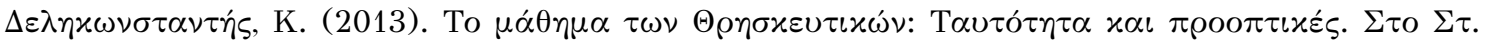

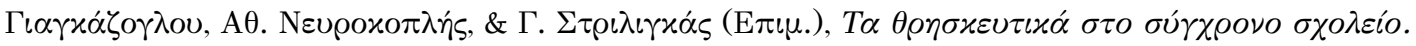

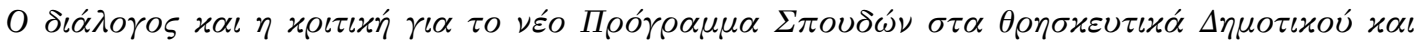

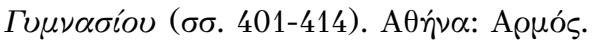

Fornell, C., Tellis, G. J., \& Zinkhan, G. M. (1982). Validity assessment: A structural equations approach using partial least squares. $\Sigma \tau$ o B. J. Walker, et al. (E $\pi \iota$.), An assessment of marketing thought $\mathcal{E}$ practice ( $\sigma \sigma$. 405-409). Chicago: American Marketing Association.

Gaskin, J. (2013). SEM series part 2: Data screening. Gaskination's Statistics.

Hair, J. F., Black, W. C., Babin, B. J., \& Anderson, R. E. (2010). Multivariate data analysis: International version (7th Ed). New Jersey: Pearson.

Hair, J. F., Black, W. C., Babin, B. J., Anderson, R. E., \& Tatham, R. L. (2006). Multivariate data analysis (Vol. 6). Upper Saddle River, NJ: Pearson Prentice Hall.

Hu, L., \& Bentler, P. M. (1999). Cutoff criteria for fit indexes in covariance structure analysis: Conventional criteria versus new alternatives. Structural Equation Modeling, 6, 1-55.

Kaiser, H. F. (1960). The application of electronic computers to factor analysis. Educational and Psychological Measurement 20, 141-51.

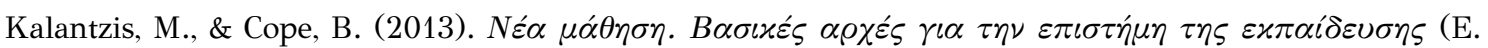

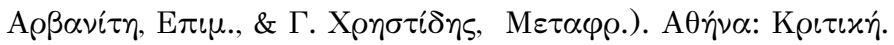

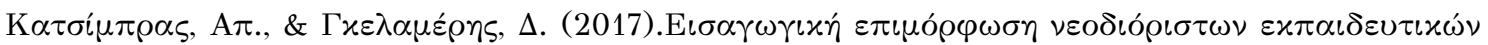

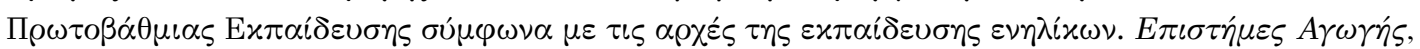
2/2018, 7-21.

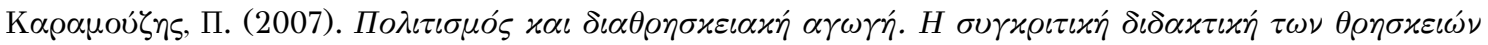

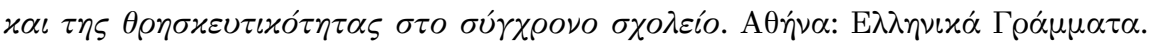

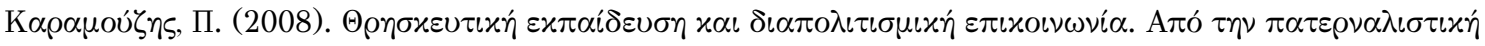

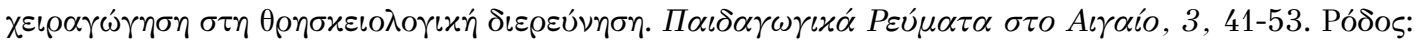

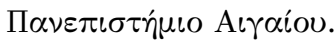

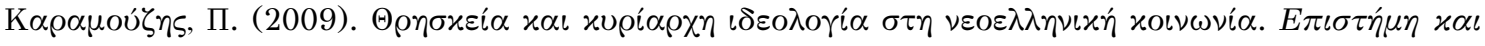

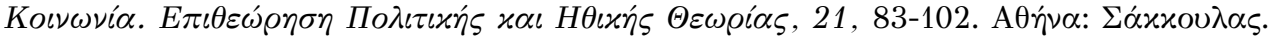

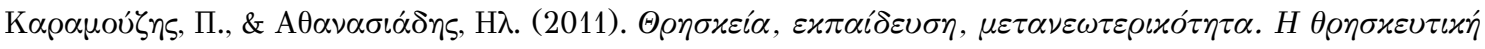

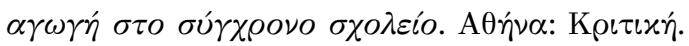




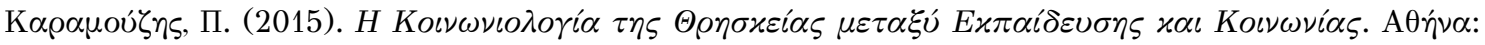

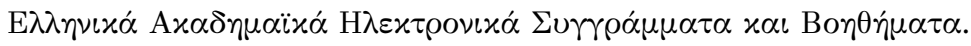

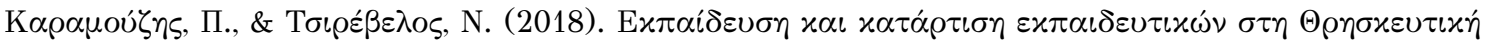

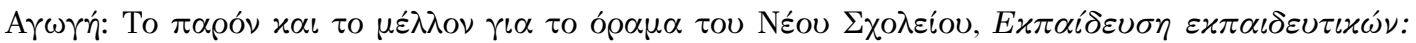

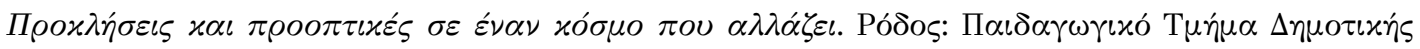

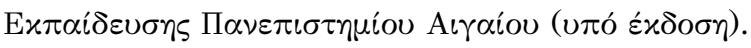

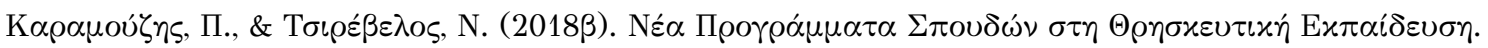

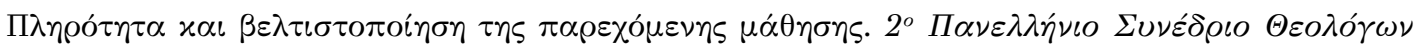

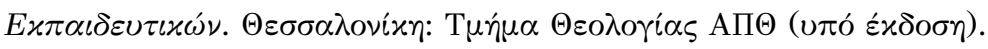

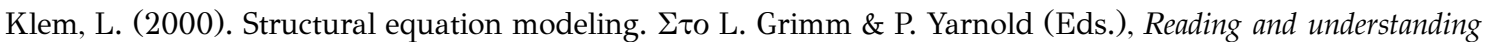
multivariate statistics, Vol. II. Washington, DC: American Psychological Association.

Kline, R. B. (2005). Principles and practice of structural equation modeling (2nd ed.). NewYork: Guilford Press.

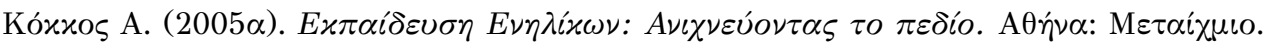

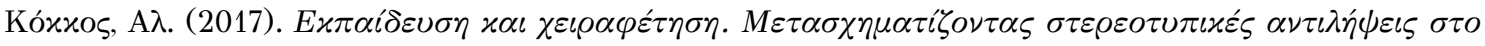

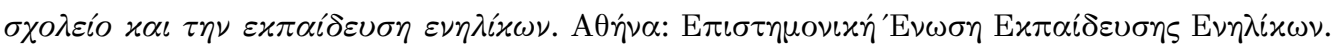

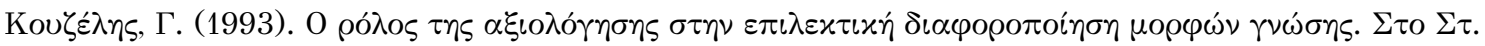

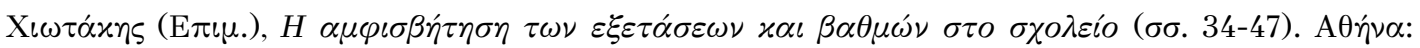
Гoทróons.

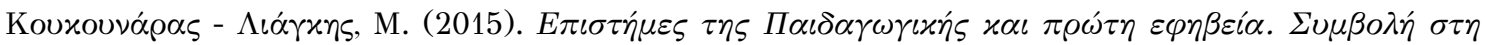

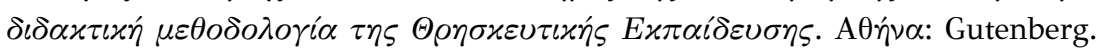

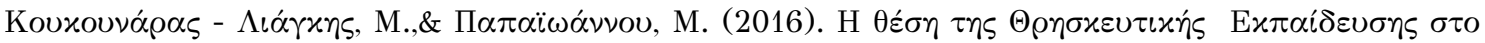

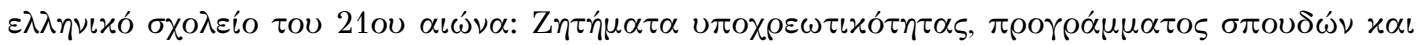

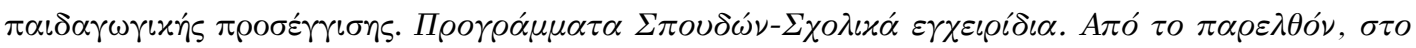

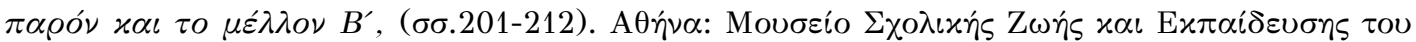

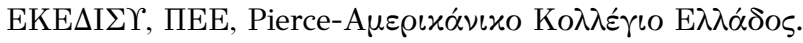

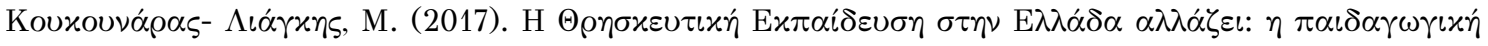

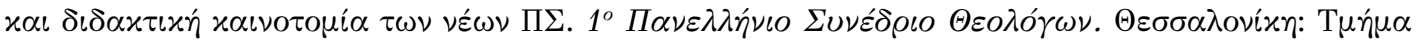

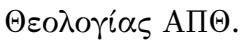

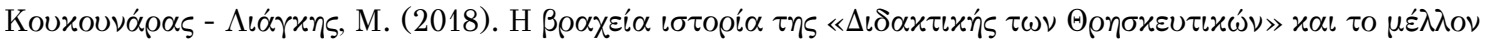

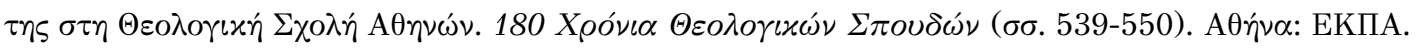

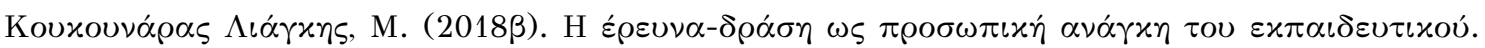

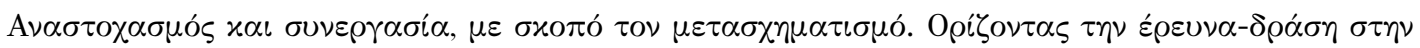

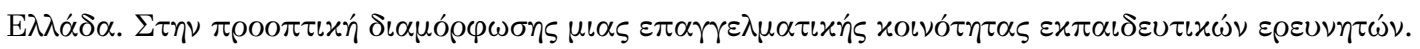

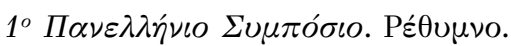

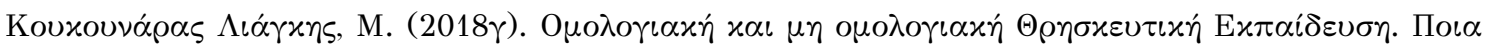

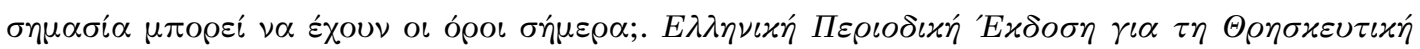

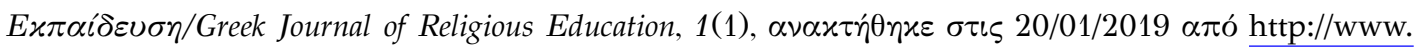
gjre.gr/images/documents/2018i/gjre2018i.pdf

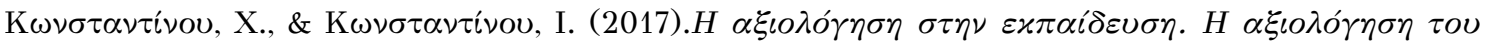

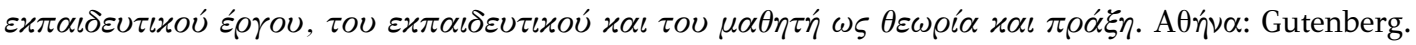

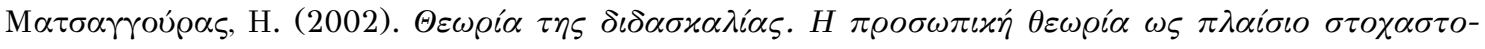

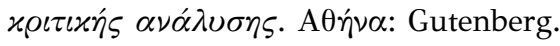




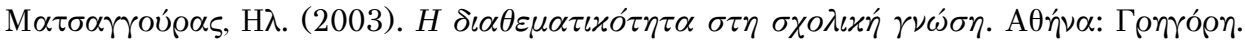

McDonald, R. P., \& Ho, M. R. (2002). Principles and practice in reporting structural equation analyses. Psychological Methods, 7(1), 64-82.

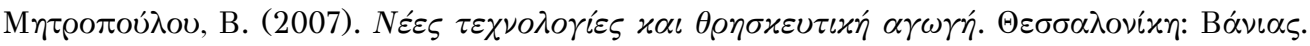

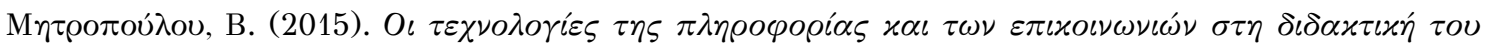
$\mu \alpha \theta \dot{\eta} \mu \alpha \tau o \varsigma \tau \omega \nu \Theta \rho \eta \sigma \varkappa \varepsilon v \tau \iota \varkappa \omega \dot{\nu}$. $\Theta \varepsilon \sigma \sigma \alpha \lambda o v i \kappa \eta$ : Ostracon Publishing.

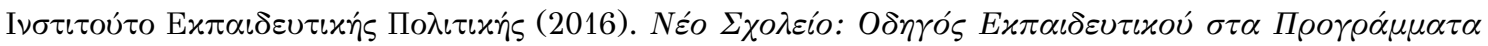

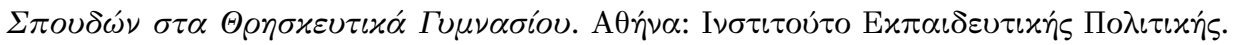

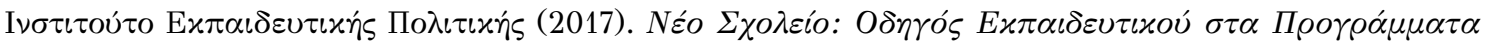

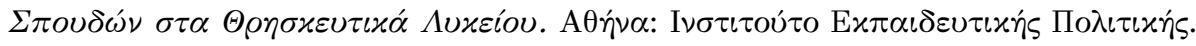

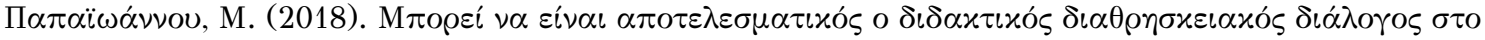

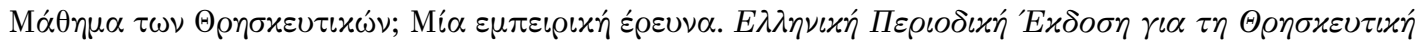

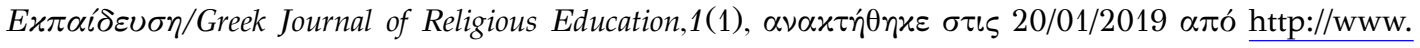
gjre.gr/images/documents/2018i/gire2018i.pdf

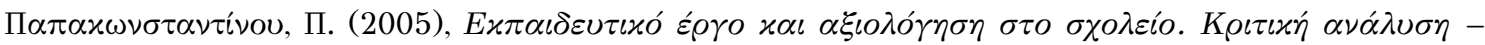

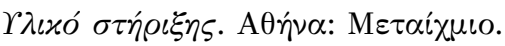

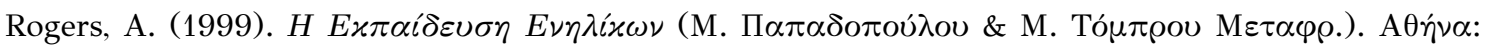

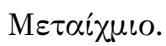

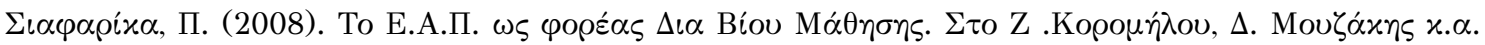

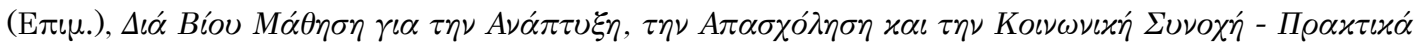

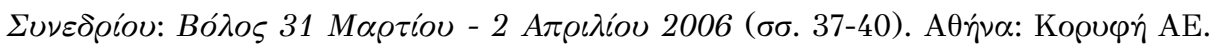

Schumacker, R. E., \& Lomax, R. G. (2010). A beginner' guide to structural equation modeling (3rd ed.). New York: Routledge.

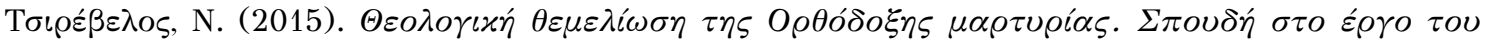

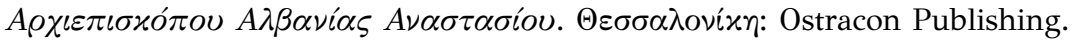

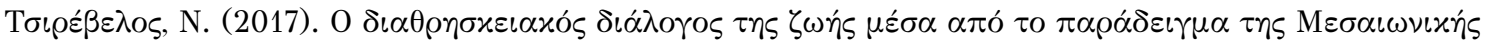

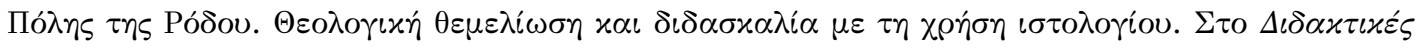

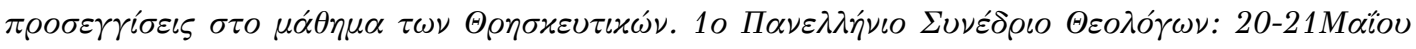

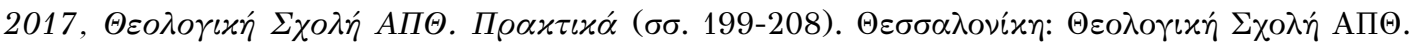
A $\alpha \propto x \tau \eta^{\prime} \theta \eta \varkappa \varepsilon \alpha \pi o ́$ http://kmaked.pde.sch.gr/site/attachments/article/1650/praktikaSYNEDRIOUTHEOLOGIAS\%20\%2018-5-18.pdf)

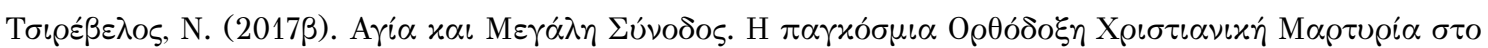

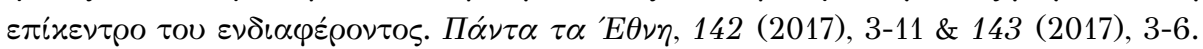

\section{$\Pi \alpha \rho \alpha ́ \rho \tau \eta \mu \alpha$}

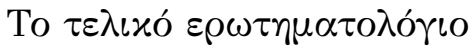

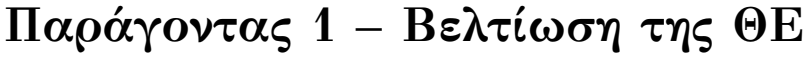

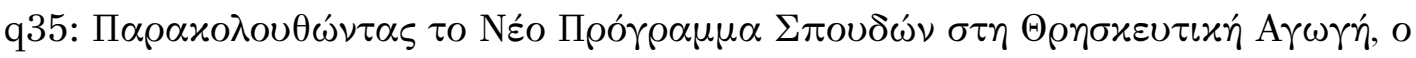

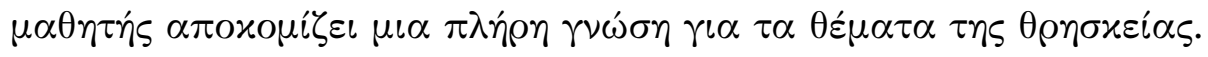




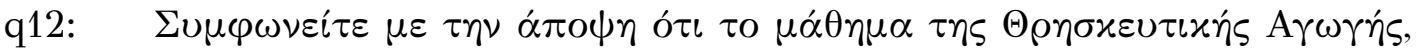

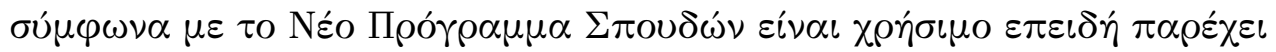

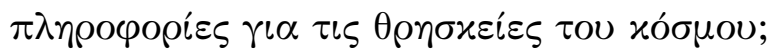

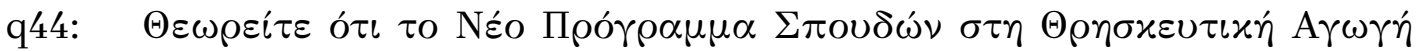

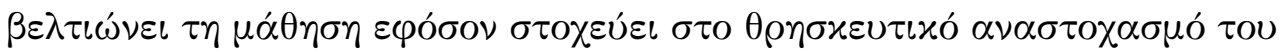
$\mu \alpha \theta \eta \tau \dot{\eta}$

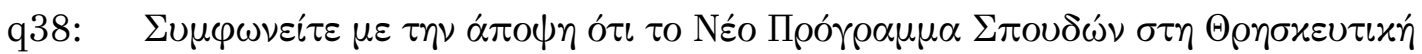

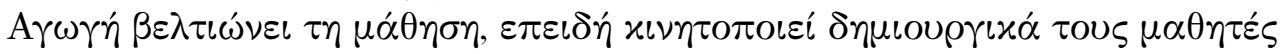

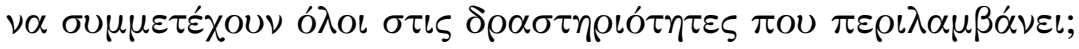

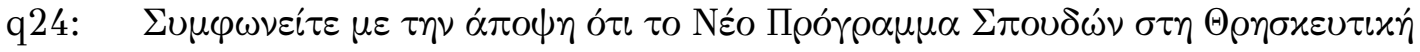

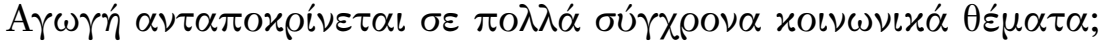

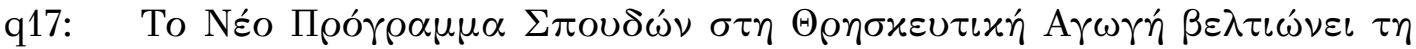

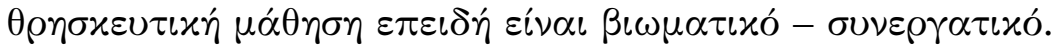

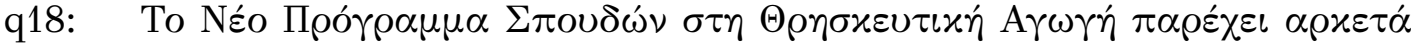

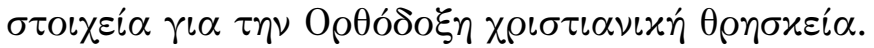

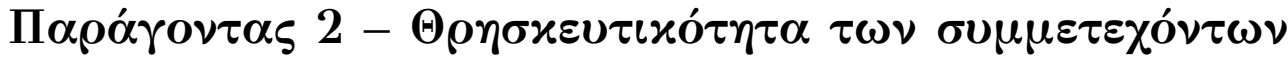

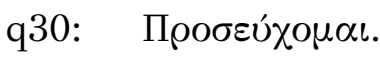

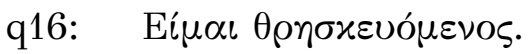

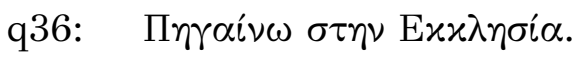

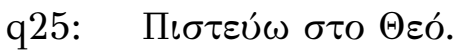

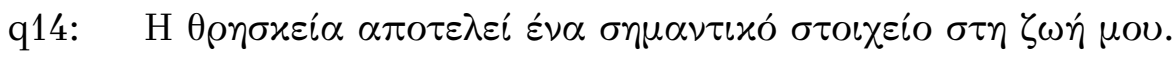

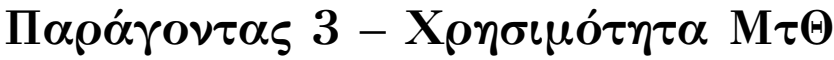

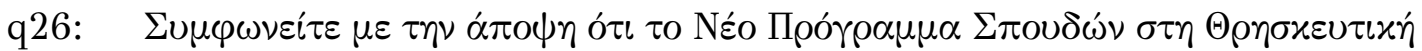

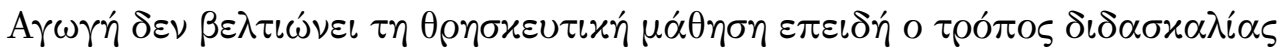

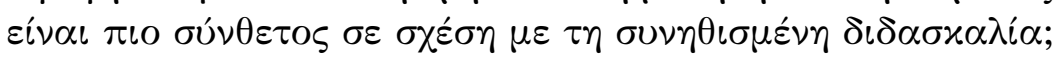

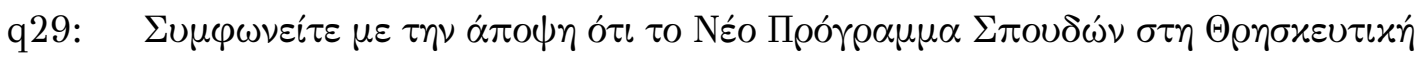

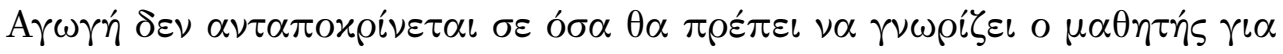
$\tau \eta \theta \rho \eta \sigma x \varepsilon i \alpha$

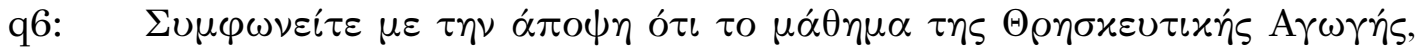

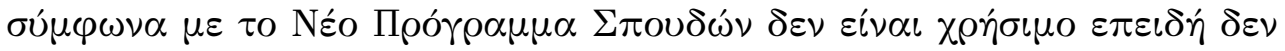

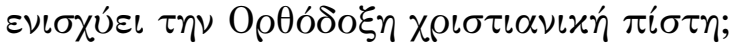

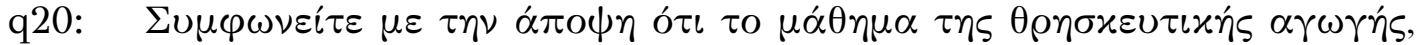

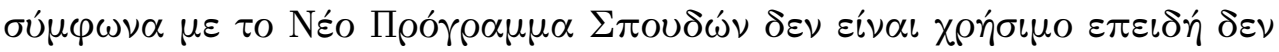

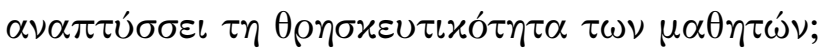




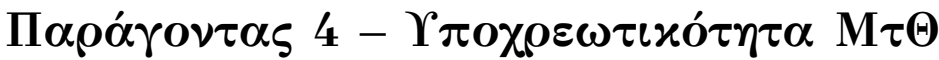

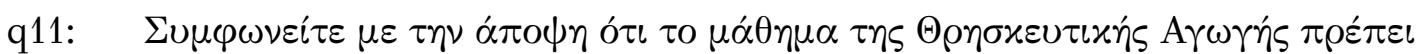

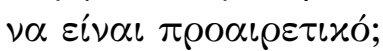

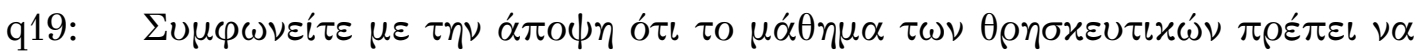
$\alpha \tau \alpha \rho \gamma \eta \theta \varepsilon i$

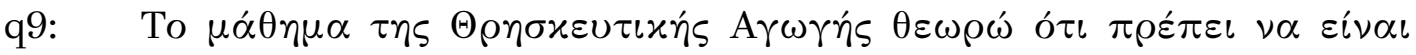

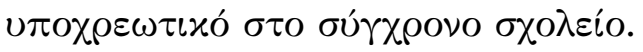

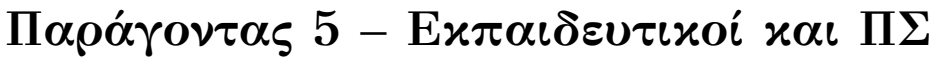

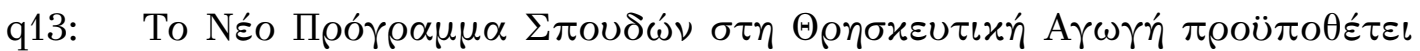

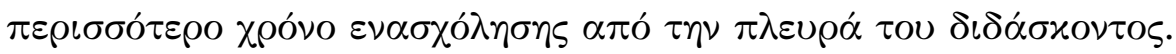

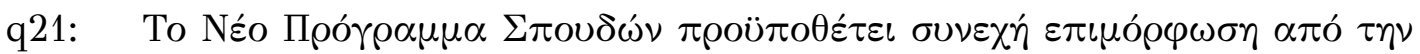

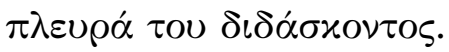

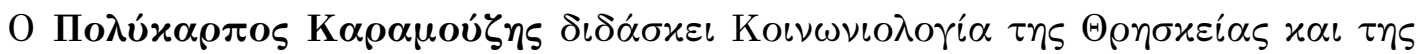

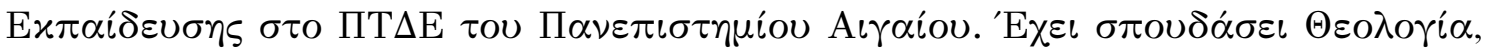

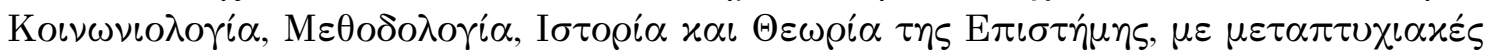

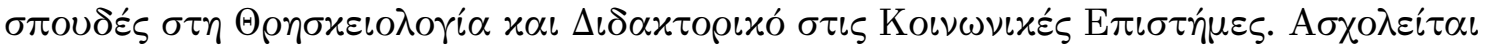

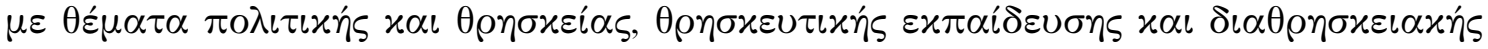

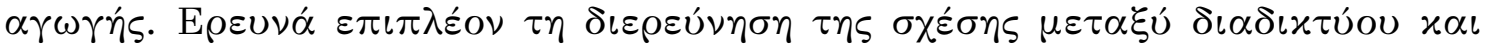

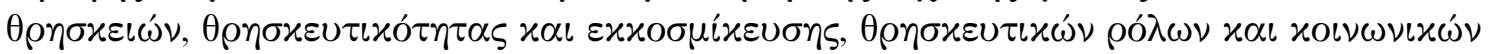
$\sigma \nu \mu \pi \varepsilon \rho \iota \varphi о \rho \omega ́ \nu$.

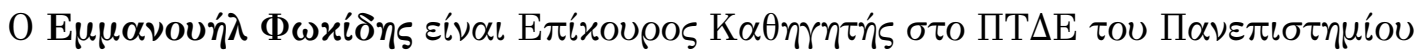

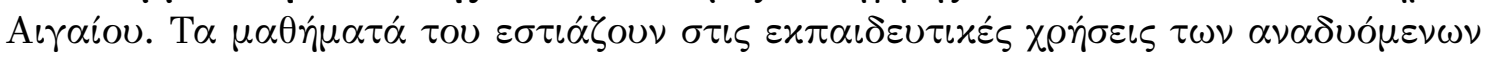

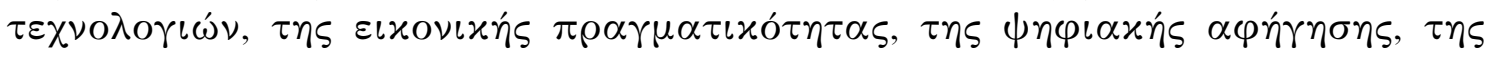

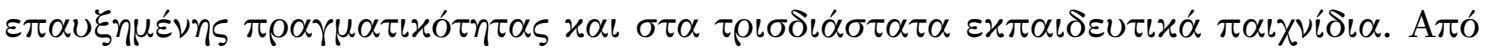

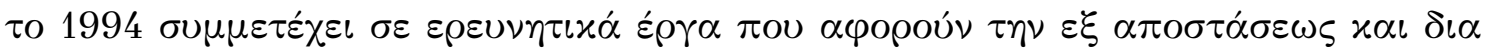

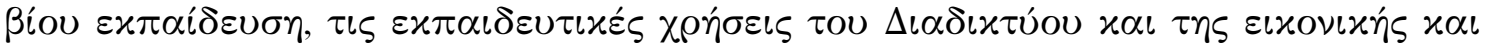

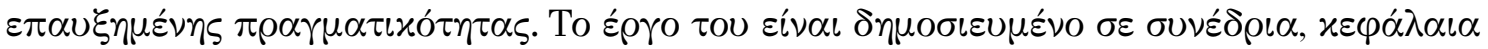

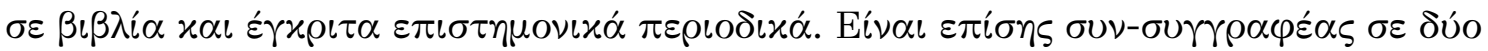
$\beta \iota \beta \lambda i \alpha$.

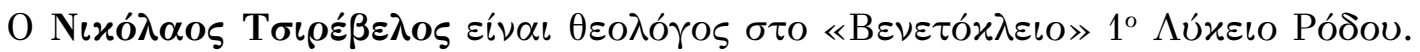

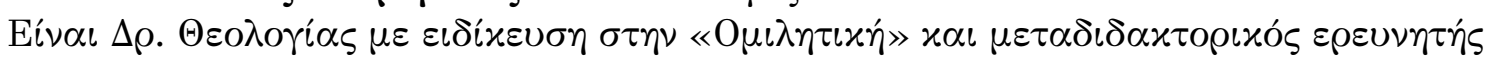

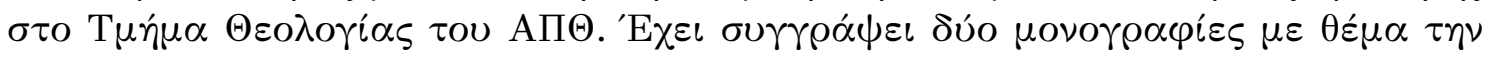

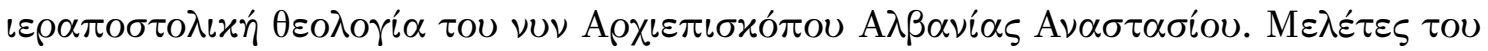

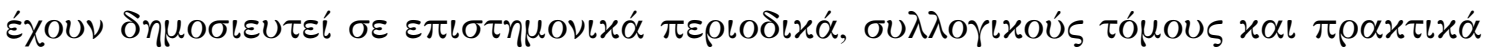

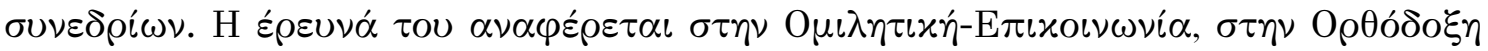

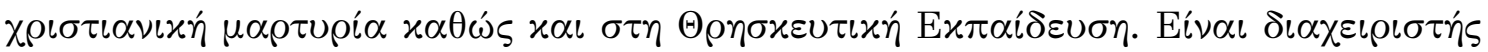

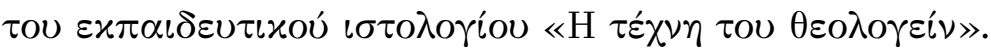

